Presented at the 1985 U.S. Particle Accelerator

School, SLAC, Stanford, California, July 1985

BNL --39057

DE87 005256

MODES OF STORAGE RING COHERENT INSTABILITIES

Jiunn-Ming Wang

National Synchrotron Light Source

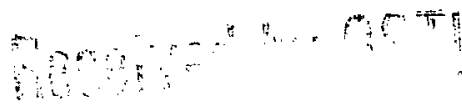

$r i \quad \therefore \quad i d i r$

\author{
Brookhaven National Labcratory \\ Upton, New York 11973
}

December 1986

DISCLAIMER

This report was prepared as an account of work sponsored by an agency of the United States Government. Neither the United States Government nor any agency thereof, nor any of their employees, makes any warranty, express or implied, or assumes any legal liability or responsibility for the accuracy, completeness, or usefulness of any information, apparatus, product, or process disclosed, or represents that its use would not infringe privately owned rights. Reference herein to any specific commercial product, process, or service by trade name, trademark, manufacturer, or otherwise does not necessarily constitute or imply its endorsement, recommendation, or favoring by the United States Government or an:-j agency thereof. The views and opinions of authors expressed herein do not necessarily state or reflect those of the United States Government or any agency thereof. 
MODES OF STORAGE RING COHERENT INSTABILITIES

Jiunn-Ming Wang
NSLS, Brookhaven National Laboratory, Upton, NY 11973
TABLE OF CONTENTS

1. Introducion

I. Longitudinal Instabilities .......................

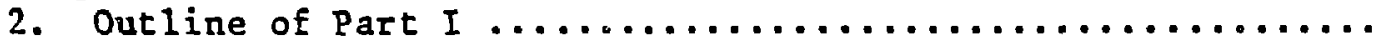

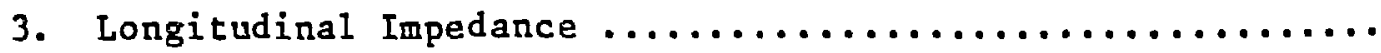

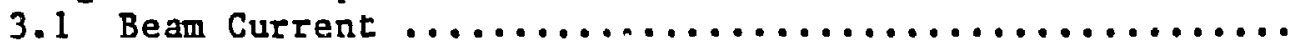

3.2 Impedance .............................

4. Coasting Beam Longitudinal Instability ...............

4.1 Density Modulation and Perturbed Particle

Displacement ............................

4.2 Equation of Motion and Dispersion Relation ..........

4.3 Solution of Dispersion Relation (without Landau

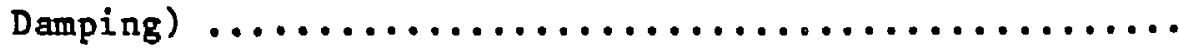

4.4 Solution of Dispersion Relation (with Landau Damping)

5. Bunched Beam Longitudinal Microwave Instability ......... 5.1 Line Eensity Modulation for a Bunched Beam ..........

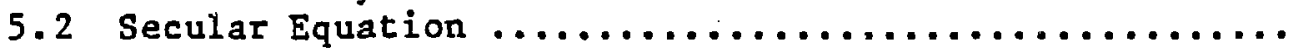

5.3 Instability Due to a High q Impedance .............

5.4 High Frequency Instability Due to a Broad Band

Impedance

6. Robinson Instability

7. Vlasov Equation for Single Bunch Longitudiral

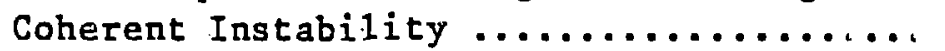

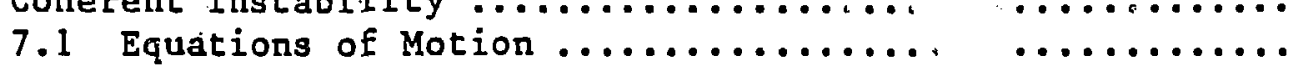

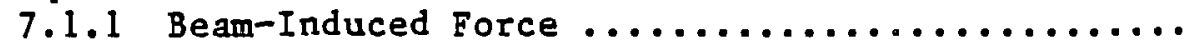

7.1.2 Linear Approximations of Equation of Motion

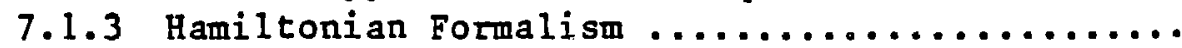

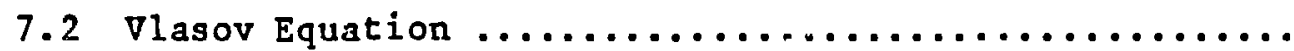

8. Synchrotron Modes ............................

9. Longitudinal strong Coupling - Short Bunch Case .........

10. Longitudinal Strong Coupling - Long Bunch Case ..........

11. Longitudinal Symetric Coupled Bunch Modes .............

II. Transverse Instabilities ........................

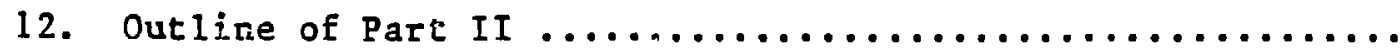

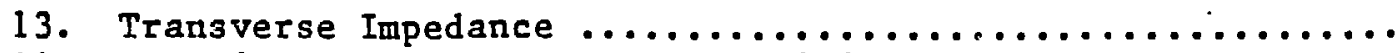

14. Coasting Beam Transverse Instability .................

14.1 Equation of Motion and Dispersion Relation ..........

14.2 Solution of Dispersion Relation (Without Landau

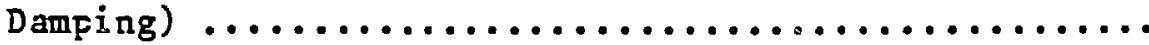

14.3 Solution of Dispersion Relation (With Landat Damping)

15. Bunched Beam Transverse Microwave Instability ...........

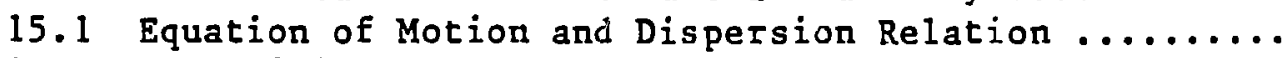

15.2 Instability Due to a High q Impedance .............

15.3 High Frequency Instability due to a Broad

Band Impedance 
16. Vlasov Equation for Single Bunch Transverse Coherent Instabilicy ..............................

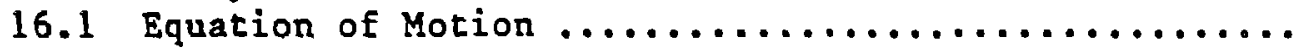

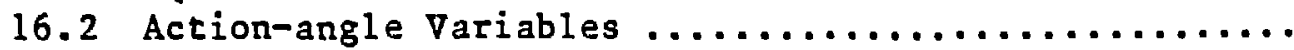

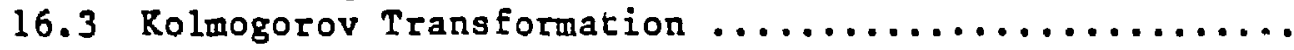

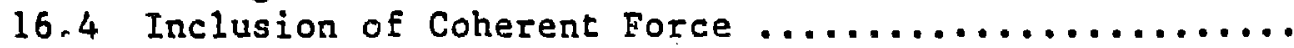

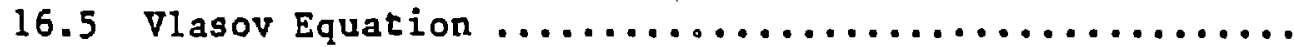

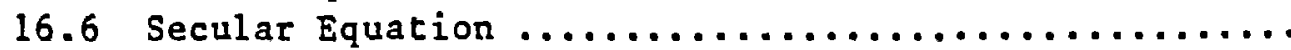

17. Head-tail Mode ..............................

18. Transverse Strong Coupling - Short Bunch Case ...........

19. Transverse Strong Coupling - Long Bunch Case ............

20. Transverse Symetric Coupled Bunch Modes .............. Appendix A Mathematical Formulae ........................ Appendix B Weak Focusing Synchrotron ..................... B.1 Expansion of Hamiltonian ...................

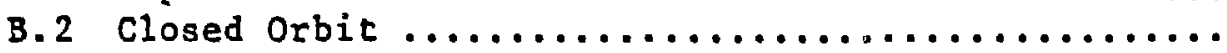
B.3 A Transformation of the Synchrotron Variable ..... Appendix C Panofsky-Wentzel Theorem ...................... Appendix D Nassibian-Sacherer Relation ....................

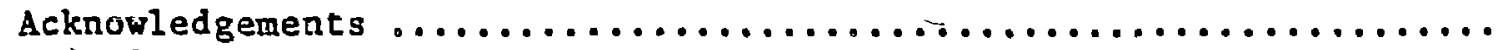
Principal Symbols $\ldots \ldots \ldots \ldots \ldots \ldots \ldots \ldots \ldots \ldots \ldots \ldots \ldots \ldots \ldots \ldots \ldots \ldots \ldots \ldots$ References 


\title{
MODES OF STORAGE RING COHERENT INSTABILITIES
}

\author{
Jiunn-Ming Wang \\ NSLS, Brookhaven National Laboratory, Upton, NY 11973
}

\section{INTRODUCTION}

In a synchrotron, the charged beam particle simultaneously carries on various oscillations with their characteristic frequencies: the revolution frequency, the betatron frequency, and, for a bunched beam, the synchrotron frequency. Ideally these oscillations are incoherent; that is, the phases of the oscillations of different particles are not correlated. However, partial correlation inevitably occurs because of the inherent fluctuation of the beam (shot noise) or external noise. Then the beam generates electromagnetic fields with the characteric frequency or its harmonics, and the EM fields act back on the beam.

When two systems oscillating with the sane frequency interact, the coupling induces a frequency shift; this ia called the coherent frequency shift in the context of these lectures. If the interaction is such that the two oscillations reinforce each other, coherent instability occurs. The coherent frequency shift corresponding to instabil-ity is a complex number. We use the convention whereby the coherent frequency (characteristic frequency + coherent frequency shift) with positive imaginary part corresponds to instability, and the negative imaginary part to damping. The imaginary part of the coherent frequency is called the growth rate; the inverse of the growth rate is the growth time.

The number of possible modes of coherent motion equals the number of degrees of freedom of the beam, which is three times the number of beam particles. Luckily, most of these modes are stable. We do not attempt to cover all the known coherent instabilities in these lectures; some topics not covered here are discussed in other review articles. $1-5$

We shall work within linear approximation, i.e., to first order in interaction between the beam and the EM field it generates. To this order of approximation, the problem of coherent motion can be formulated as a linear eigenvalue problem, where the eigenvalue yields information about the coherent frequency, and the eigenvector describes the beam distortion corresponding to that eigenmode.

We divide the coherent oscillation of the beam into two classes, longitudinal and transverse, and discuss the longitudinal modes of coherent instabilities in Part $I$ and the transverse in Part II.

Let us close this introduction by defining and explaining some notations and conventions which are consistently adopted throughout these lectures.

The independent variable of the particle motion is time $t$. To describe the position of the particle, the Serret-Frenet coordinate system ${ }^{6}, 7(x, y, s)$ with $\hat{x} \times \hat{y}=\hat{s}$ is used (the carat indicating a unit vector.) 
$E_{0}, P_{0}, \omega_{0}$ : nominal energy, momentum, and angular revolution frequency, respectively, of the ring.

$\beta_{o}$ : nominal particle velocity in units of $c$.

$$
\theta=s / R, \quad \phi=\theta-\omega_{0} t,
$$

where $R$ is the average ring radius. $\theta$ as well as $s$ describes the position of the particle relative to the storage ring. $\phi$ describes the particle position relative to a prescribed reference particle rotating around the ring with nominal revolution Erequency $\omega_{0} / 2 \pi$.

With $\mathrm{N}$ denoting the total number of particles in the beam, the average current is

$$
I_{a v}=e^{\omega}{ }_{0} N / 2 \pi
$$

We define

$\delta=\left(\mathrm{p}-\mathrm{p}_{0}\right) / \mathrm{P}_{0}, \quad \varepsilon=\left(E-E_{0}\right) / \mathrm{E}_{0}, \quad W=\left(E-E_{0}\right) / W_{0}$,

where $\delta$ and $\varepsilon$ are, respectively, the fractional momentum and energy deviation of the particle. If the dynamics of the particle are described in terms of the standard Hamiltonian of the Lorentz force equatio:s, then $W$ is the canonical momentum conjugate to the canonical coordinate $\phi$. Also

$$
\eta=\gamma_{t}^{-2}-\gamma^{-2}, \quad \bar{\eta}=n \omega_{0}^{2} /\left(\beta_{0}^{2} E_{0}\right),
$$

where $\gamma_{t}$ is the transition energy in units of the rest energy.

The following relations are often used:

$$
\begin{aligned}
& \delta=\varepsilon / \beta_{0}^{2}=\omega_{0} W /\left(B_{0}^{2} E_{0}\right), \\
& \dot{\phi}=-n \omega_{0} \delta=-\bar{n} \omega,
\end{aligned}
$$

where a dot above a symbol indicates a time derivative. $\phi$ is, from Eq. (1-1), the angular revolution frequency deviation.

\section{LONGITUDINAL INSTABILITIES}

\section{OUTLINE OF PART I}

We start with a discussion in Section 3 of the longitudinal electric field $\mathscr{E}$ induced by the current $I . \mathscr{E}$ and $I$ are related linearly by a "transfer function" called longitudinal impedance. We then treat various modes of longitudinal coherent instabilities.

Section 4 concerns the coasting beam coherent instability. This instability is the easiest mode to study because the translational invariance of the unperturbed beam causes each Fourier component of 
the perturbed line density to correspond to an eigenmode, so that each coherent mode is characterized by a harmonic number $n$ of the revolution trequency. We call $n$ the revolution mode number.

Boussard ${ }^{9}$ has conjectured by intuitive reasoning that, if the perturbing EM fields have wavelangth short compared with the bunch length, and if the growth rate of the instability is much greater than the synchrotron frequency, then the bunched beam coherent instability looks like that of a coasting beam. Such bunched beam instabilities are, for a historical reason, called microwave instabilities. Messerschmidt and Monthlo reasoned that, since the growth rate is much greater than the synchrotron frequency, the synchrotron frequency is irrelevant, and we can set the angular synchrotron frequency $\omega_{s}=0$ in discussing the microwave instability. This is done in Section 5 .

Sections 6 to 10 concern single-bunch longitudinal coherent instabilities with $\omega_{s}$ fully taken into account.

Robinson instability, 11-14,2 treated in Section 6 , is the simplest of the longitudinal coherent modes that involve the synchrotron frequency. In this mode, the bunch is displaced rigidly from the synchronous point and oscillates with the synchrotron frequency about this fixed point.

In Section 7, the vlasov equation is formulated, and a method $15-19$ of solving it is developed which is followed in the rest of Part I.

Section 8 treats the synchrotron modes, $20-23,19$ which take the possible bunch shape distortion fully into account. An eigenmode is characterized by a harmonic number $\mu$ of the synchrotron frequency; $\mu$ describes the degree of the bunch shape distortion and is called the synchrotron mode number. The Robinson mode is a synchrontron mode with $\mu=1$,

When the coupling between the bunch current and the EM fields becomes big, the synchrotron modes cease to be eigenmodes. 24 In section 9 , a method $26,17-19,27$ of treating the synchrotron mode coupling in the case of a small bunch is discussed which takes advantage of the fact that only a few of the synchrotron modes can contribute in such a case.

If the bunch is longei than the wavelengths of the perturbing EM fields, and if the interaction of the beam current and the EM fields is large, then all the synchrotron modes couple, and this leads to microwave instability. We treat 15-19 in Section 10 the microwave instability without setting $\omega_{s}=0$.

In Section 11, we consider how the presence of many bunches affects the coherent motion of the beam, and we treat the longitudinal symetric coupled bunch modes. $21-23$

Throughout these lectures, we ignore Landau damping due to synchrotron frequency spread. 34

\section{LONGITUDINAL IMPEDANCE}

The EM fields responsible for the coherent instability are solutions of Maxwell equations, where the source terms are the charge and the current densities, and the boundary conditions are determined by the devices surrouding the beam: beam chamber, rf cavity, bellows, etc. 
The component of the EM fields responsible for the longitudinal instability is the longitudinal component $\mathscr{E}$ of the electric field. The longitudinal impedance function $Z_{n}(\omega)$ conveniently relates the electric field $\mathscr{E}$ to the beam current.

\subsection{Beam Current}

We use $\rho(\phi, t)$ and $\Psi(\phi, \dot{\phi}, t)$, to denote the particle distribution functions in $\phi$-space and in $(\phi, \phi)$-space, respectively. They are related by

$$
\rho(\phi, t)=\int d \dot{\phi} \Psi(\phi, \dot{\phi}, t)
$$

We normalize $\rho$ and $\Psi$ to 1 :

$$
\int_{0}^{2 \pi} d \phi \rho(\phi, t)=\int_{0}^{2 \pi} d \phi \int_{-\infty}^{\infty} d \dot{\phi} \Psi(\phi, \dot{\phi}, t)=1 .
$$

The angular velocity of a particle is $\omega_{0}+\dot{\phi}$; hence the beam current is

$$
\text { - } I(\theta, t)=\operatorname{eN} \int d \dot{\phi}\left(\omega_{0}+\dot{\phi}\right) \Psi(\phi, \dot{\phi}, t) \text {. }
$$

If we ignore the angular revolution frequency deviation $\dot{\phi}$ relative to $\omega_{0}$, this equation becomes

$$
I(\theta, t)=2 \pi I_{a v} \rho(\phi, t) \text {. }
$$

Recall that $\theta$ and $\phi$ are, in our notation, always related by Eq. (1-1). In terms of the Fourier components of $I, \Psi$ and $\rho$, defined by

$$
\begin{aligned}
& I(\theta, t)=\sum_{n=-\infty}^{\infty} \int_{-\infty}^{\infty} d \omega I_{n}(\omega) e^{i n \theta-i \omega t} \\
&=\sum_{n} d \Omega I_{n}\left(n \omega_{0}+\Omega\right) e^{i n \phi-i \Omega t}, \\
& \Psi(\phi, \dot{\phi}, t)=\sum_{n} d \Omega \Psi \\
& n
\end{aligned}
$$

and

$$
\rho(\phi, t)=\sum_{n} d \Omega \rho_{\pi}(\Omega) e^{i n \phi-\Omega t},
$$

Eqs. (3-3a) and (3-3b) can be written, respectively, as

$$
I_{n}\left(n \omega_{0}+\Omega\right)=2 \pi I_{a v} \int d \dot{\phi}\left(1+\frac{\dot{\phi}}{\omega_{0}}\right) \Psi_{n}(\dot{\phi}, \Omega),
$$


and

$$
I_{n}\left(n \omega_{0}+\Omega\right)=2 \pi I_{a v} \rho_{n}(\Omega) \text {. }
$$

We always use $\omega$ as the variable Fourier-conjugate to $t$ if $\theta$ is kept fixed, and use $\Omega$ if $\phi$ is fixed.

Note that the angular phase velocity of the $(n, \Omega)$ component in Eqs. (3-4) and $(3-5)$ is $\omega_{0}+\Omega / n$. The beam can sustain this component only if the phase velocity is close to the particle velocity; i.e.,

$$
\left|\Omega / n \omega_{0}\right| \ll 1 \text {. }
$$

We therefore assume that $I_{n}\left(n \omega_{0}+\Omega\right), \rho_{n}(\Omega)$, and $\Psi_{n}\left(\dot{\phi}, \Omega_{1}\right)$ are small unless Eq. $(3-7)$ is satisfied.

\subsection{Impedence}

The longitudinal electric field can in general be expressed in terms of $I(\theta, t)$ as

$$
\mathscr{E}(\theta, t)=\int_{0}^{2 \pi} d \theta \cdot \int_{-\infty}^{\infty} d t^{\prime} \bar{G}\left(\theta, \theta^{\prime}, t-t^{\prime}\right) I\left(\theta^{\prime}, t^{\prime}\right)
$$

The Green's function $\bar{G}$ depends on $\theta$ and $\theta$ ' separately, since the environment is generally not invariant under azimuthal tranlation.

In terms of the Fourier component of the Green's function defined by

$$
\bar{G}\left(\theta, \theta^{\prime}, t\right)=\sum_{m, n} \int d \omega \bar{G}_{m, n}(\omega) e^{i m \theta-i n \theta^{\prime}-i \omega t},
$$

the longitudinal electric field induced by the $(n, \Omega)$ component of the current $(3-4 b)$ is

$$
\mathscr{E}(\theta, t)=4 \pi^{2} \sum_{m} \bar{G}_{m, n}\left(\pi \omega_{0}+\Omega\right) I_{n}\left(n \omega_{0}+\Omega\right) e^{i m \theta-i\left(n \omega_{0}+\Omega\right) t} .
$$

The angular phase velocity of the m-th term above is

$$
\frac{\mathbf{n}}{\mathbf{m}} \omega_{0}+\frac{\Omega}{\mathrm{m}}
$$

Therefore, this component cannot interact coherently or resonate with the beam oscillation unless $m=n$. As a consequence, we discard the terms in Eq. (3-10) with $m \neq n$ and write

$$
\bar{G}_{m, n}(\omega)=-\frac{1}{8 \pi^{3} R} \delta_{m, n} z_{n}(\omega)
$$


where $\delta_{\mathbb{m}, \mathbb{n}}$ is Rronecker's $\delta$. The relevant part of Eq. (3-10) becomes*

$$
\mathscr{E}(\theta, t)=-\frac{1}{2 \pi R} I_{n}\left(n \omega \omega_{0}+\Omega\right) z\left(n \omega_{0}+\Omega\right) e^{i n \theta-i\left(n \omega_{0}+\Omega\right) t} .
$$

The function $\mathrm{z}_{\mathrm{n}}(\omega)$ is the longitudinal impedance. From $(3-11)$, Eq. $(3-9)$ becomes

$$
\bar{G}(\theta, \theta \cdot, t)=-\frac{1}{2 \pi R} G(\theta-\theta \cdot, t)
$$

where

$$
G(\theta, t)=\frac{1}{4 \pi^{2}} \sum_{n=-\infty}^{\infty} \int d w z_{n}(\omega) e^{i n \theta-i \omega t}
$$

and Eq. (3-8) becomes

$$
\mathscr{E}(\theta, t)=\frac{1}{2 \pi R} \int_{0}^{2 \pi} d \theta^{\prime} I_{-\infty}^{\infty} d t^{\prime} G\left(\theta-\theta^{\prime}, t-t^{\prime}\right) I\left(\theta^{\prime}, t^{\prime}\right),
$$

or ${ }^{28}$

$$
\begin{aligned}
\mathscr{E}(\theta, t) & =-\frac{1}{2 \pi R} \sum_{n=-\infty} \int d \omega I_{n}(\omega) Z_{n}(\omega) e^{i n \theta-i \omega} \\
& \left.=-\frac{1}{2 \pi R} \sum_{n} \int d \Omega I_{n}(n \omega)_{0}+\Omega\right) Z_{n}\left(n \omega \omega_{0}+\Omega\right) e^{i n \phi-i \Omega t} .
\end{aligned}
$$

Equation (3-14) represents the solution of Maxwell's equation, and it is one of the foundations of all the following discussion in Part I of these lectures.

We now discuss the constraints on $2_{n}(w)$ which follow from the causality condition,

$$
G(\theta, t)=0 \quad \text { if } \quad t<0
$$

From Eq̧s. $(3-13)$ and $(3-15)$,

$$
z_{n}(\omega)=\int_{0}^{2 \pi} d \theta \int_{0}^{\infty} d t G(\theta, t) e^{-i n \theta+i \omega t} \text {. }
$$

*The approximation used here is similar to the familiar smooth approximation we use in discussing the longitudinal phase focusing. Since the rf cavity is localized, the rf voltage can be represented by a superpositon of propagating waves of longitudinal electric field with angular phase velocity $\mathrm{h}_{\mathrm{o}} / \mathrm{n}, \mathrm{n}=0, \pm 1, \pm 2, \ldots$. The smooth approximation consists of keeping only the wave with its phase velocity equal to the particle velocity; namely, the wave with $n=+h$. 
The function $Z_{n}(\omega)$ can be analytically continued to the upper half of the complex $\omega$ plane through this equation. If follows from Eq. (3-16) that

$$
z_{n}^{*}(\omega)=z_{-n}(-\omega *) .
$$

Let us now separate $z_{n}(\omega)$ into the real part and the imaginary part,

$$
z_{n}(\omega)=\mathscr{R}_{n}(\omega)+i x_{n}(\omega) .
$$

The real part $\mathscr{R}_{\mathrm{n}}(\omega)$ is usually called the resistive part and the imaginary part $\mathrm{x}_{\mathrm{n}}(\omega)$, the reactive part. $\mathrm{x}_{\mathrm{n}}(\omega)$ is said to be capacitive (inductive) if it is positive (negative) for $\omega>0$. The symmetry properties for $\mathscr{R}_{\mathrm{n}}$ and $\mathrm{x}_{\mathrm{n}}$ are, from Eq. (3-17),

$$
\mathscr{R}_{\mathbf{n}}(\omega)=\mathscr{R}_{-\mathbf{n}}(-\omega *) \text {, }
$$

and

$$
x_{n}(\omega)=-x_{-n}(-\omega *) .
$$

The contribution to $z_{\mathfrak{n}}(\omega)$ from the smooth resistive beam pipe is independent of the subscript $n$. For example, a round baam pipe of radius $b$ and conductivity $\sigma$ contributes an amount 29

$$
(1-i) \sqrt{\frac{\omega \rho_{0}}{2 \sigma}} \frac{R}{b}
$$

to $z_{n}(\omega)$. One can also show that, under the smooth approximation, the contribution from a localized source of impedance is also independent of the subscript $n$.

Exercise: Prove the last statement.

We show in the following that the resistive part of the impedance from a passive device is positive.

Let us take the current of the form

$$
I(\theta, t)=I_{n}(\omega) e^{i n \theta-i \omega t}+I_{n}^{*}(\omega) e^{-i n \theta+i \omega * t} .
$$

We include two terms here to ensure that the current is real. The electric field induced by this current is

$\mathscr{E}(\theta, t)=\frac{-1}{2 \pi R}\left(I_{n}(\omega) z_{n}(\omega) e^{i n \theta-i \omega t}+I_{n}^{*}(\omega) z_{-n}\left(-\omega^{*}\right) e^{-i n \theta+i \omega * t}\right)$. 
The rate of energy gain of the beam from is

$$
\begin{aligned}
\frac{d E_{\text {beam }}}{d t} & =R \int_{0}^{2 \pi} d \theta(\theta, t) I(\theta, t) \\
& =-\left|I_{n}(\omega)\right|^{2}\left(z_{n}(\omega)+z_{-n}\left(-\omega^{*}\right)\right) e^{+i(\omega *-\omega) t} \\
& =-2\left|I_{n}(\omega)\right|^{2} \mathscr{R}_{n}(\omega) e^{i\left(\omega^{*}-\omega\right) t},
\end{aligned}
$$

where Eq. (3-17) has been used. Note that the exponential factor in Eq. $(3-22)$ is positive.

When the beam passes through a passive device, it cannot gain energy. Therefore,

$$
\frac{d E_{\text {beam }}}{d t} \leq 0 \text {, }
$$

or, from Eq. (3-22;,

$$
\mathscr{R}_{\mathrm{n}}(\omega) \geq 0
$$

We conclude this section by remarking that the range in frequency where $z_{n}(\omega)$ is effective is called the bandwidth of the impedance, and the inverse of the bandwidth is called the wakelength. The wakelength is the length of time during which the electric field $\mathscr{E}$ generated by a very short pulse of the current source remains appreciable.

The wakelength can also be understood to be the coherent length; it is the time interval during which the frequency components of the electric field"induced by a current pulse remain partially coherent with each other.

\section{COASTING BEAM LONGITUDINAL INSTABILITY}

\subsection{Density Modulation and Perturbed Particle Displacement}

The longitudinal electric field $\mathscr{E}$ responsible for the longitudinal coherent instability is induced by the perturbation of the beam current which is in turn related to the beam density modulation (see Eqs. $(3-14)$ and $(3-3))$. We establish here the relationship, to first order in the particle displacement, between the line density modulation and the displacement of the particles from their respective nominal positions in the beam.

The angular revolution frequency of a particle with fractional momentum deviation $\delta=\left(p-p_{0}\right) / p_{0}$ is $\omega_{0}(1-\eta \delta)$. Therefore, the unperturbed position of the $j-t h$ particle can be written as $\phi_{o j}-\omega_{0} \eta \delta_{j} t$. In the presence of an azimuthal displacement $\zeta$ of the particle due to perturbation, the particle position is

$$
\phi_{j}=\phi_{0 j}-\omega_{0} \eta \delta_{j} t+\zeta\left(\phi_{0 j}-\omega_{0} \eta \delta_{j} t, \delta_{j}, t\right),
$$


where the perturbed displacement $\zeta$ is taken to be a function of the unperturbed position, the momentum deviation, and time. The normalized particle distribution function in $(\phi, \delta)$ space corresponding to Eq. $(4-1)$ is

$$
\begin{aligned}
\frac{1}{N} \sum_{j=1}^{N} \delta_{p}\left(\phi-\phi_{o j}+\omega_{o} \eta \delta_{j} t-\zeta\left(\phi_{o j}-\omega_{o} \eta \delta t, \delta, t\right)\right) \delta\left(\delta-\delta_{j}\right) \\
=\frac{1}{N} \sum_{j} \delta_{p}\left(\phi-\phi_{o j}+\omega_{o} \eta \delta j t\right)-\frac{1}{N} \sum_{j} \zeta\left(\phi_{o j}-\omega_{o} \eta \delta_{j} t, \delta j, t\right) \\
\quad \cdot \delta_{p}^{\prime}\left(\phi-\phi_{j}+\omega_{o} \eta \delta_{j} t\right) \delta\left(\delta-\delta_{j}\right)
\end{aligned}
$$

where $\delta_{p}$ is a periodic delta function with period $2 \pi$. The subscript $p$ for a periodic delta function will often be suppressed.

Assuming the beam particles to be uncorrelated without the perturbation $\zeta_{;}$one can write the probability density of the unperturbed particles as

$$
P\left(\phi_{\circ 1}, \delta_{1} ; \phi_{\circ 2}, \delta_{2} ; \ldots ; \phi_{\circ N}, \delta_{N}\right)=\prod_{j=1}^{N} g\left(\delta_{j}\right) \lambda\left(\phi_{\circ j} ;,\right.
$$

where the distribution functions $g$ and $\lambda$ are normalized to 1 :

$$
\int_{-\infty}^{\infty} \mathrm{d} \delta \mathrm{g}(\delta)=\int_{0}^{2 \pi} \mathrm{d} \phi \lambda(\phi)=1 \text {. }
$$

For a coasting beam

$$
\lambda(\phi)=1 / 2 \pi
$$

The distribution function $\Psi(\phi, \delta, t)$ including the perturbation is obtained by multiplying Eqs. (4-2) and (4-3) and then by integrating over $\phi_{j}$ and $\delta_{j}, j=1,2, \ldots, N$. One finds

with

$$
\Psi(\phi, \delta, t)=\frac{1}{2 \pi} g(\delta)+\Psi_{1}(\phi, \delta, t)
$$

$$
\Psi_{1}(\phi, \delta, t)=-\frac{1}{2 \pi} g(\delta) \frac{\partial}{\partial \phi} \zeta(\phi, \delta, t) .
$$

The line density is obtained by integrating $(4-6)$ and $(4-7)$ over $\delta$. It is

$$
\rho(\phi, t)=\frac{1}{2 \pi}+\rho^{(1)}(\phi, t)
$$

with 


$$
\rho^{(1)}(\phi, t)=-\frac{1}{2 \pi} \int d \delta_{g}(\delta) \frac{\partial}{\partial \phi} \zeta(\phi, \delta, t) .
$$

The Fourier components of $\rho(1)$ and $\zeta$ defined by

$$
\rho^{(1)}(\phi, t)=\sum_{n} \int_{n} \alpha \rho(\Omega) e^{i n \phi-i \Omega t}
$$

and

$$
\zeta(\phi, \delta, t)=\sum_{\Omega} \int_{n} d \Omega \zeta_{n}(\delta, \Omega) e^{i n \phi-i \Omega t}
$$

are related, from Eq. (4-9), by

$$
\rho_{n}(\Omega)=-i \frac{n}{2 \pi} \int d \delta g(\delta) \zeta_{n}(\delta, \Omega) .
$$

4.2 Equation of Motion and Dispersion Relation

The equation of motion of a particle is

$$
\frac{d^{2}}{d t^{2}} \zeta\left(\phi_{\circ j}-\omega_{0} n \delta j t, \delta_{j}, t\right)=-\frac{e n \omega_{0} c}{\delta_{0} E_{0}} \mathscr{E}(\theta, t) .
$$

The electric field $\mathscr{E}$ should be evaluated at the position of the patticle, $\theta=\omega_{0} t+\phi_{o j}-\omega_{0} n \delta j t+\zeta$. Within linear approximation of Eq. (4-13) (1inear in $\zeta$ ), we can set $\theta=\omega_{0} t+\phi_{0 j}-\omega_{0} n \delta j t$.

From Eqs. (3-14) and (3-6b), Eq. (4-13) becomes

$$
\begin{aligned}
\frac{d^{2}}{d t^{2}} \zeta\left(\phi_{0}-\omega_{0} n \delta t, \delta, t\right)= & \bar{\eta}_{e} I_{a v} \sum_{n} \int d \Omega z_{n}\left(\omega_{0}+\Omega\right) \\
& x \rho_{a}(\Omega) e^{i n\left(\phi_{0}-\omega_{0} \eta \delta t\right)-i \Omega t},
\end{aligned}
$$

where the subscript $j$ is suppressed, and $\bar{n}$ is given by Eq. $(1-4)$. Substitution of Eq. (4-11) into Eq. (4-14) yields

$$
\left(\Omega+\pi \omega_{0} n \delta\right)^{2} \zeta_{n}(\delta, \Omega)=-\bar{\eta}_{e} I_{a v} z_{n}\left(\omega_{0}+\Omega\right) \rho_{n}(\Omega) \text {. }
$$

We now eliminate $\zeta_{n}$ and $\rho_{n}$ from $(4-12)$ and $(4-15)$ and obtain the dispersion relation

$$
1=\frac{i}{2 \pi} \bar{\eta}_{e} I_{a v} n z_{n}\left(n w_{0}+\Omega\right) \int_{-\infty}^{\infty} d \delta \frac{g(\delta l}{\left(\Omega+\pi w_{0} \eta \delta\right)^{2}}
$$

or, equivalently,

$$
1=i \frac{\omega_{0}}{2 \pi \beta_{0}^{2} E_{0}} e \operatorname{av}_{n} z_{0}\left(\pi \omega_{0}+\Omega\right) \int_{-\infty}^{\infty} d \delta \frac{g^{\prime}(\delta)}{\Omega+\pi \omega_{0} n \delta} .
$$


In sumary, we have discovered that the linear eigenvalue problem represented by Eqs. (4-9) and $(4-14)$ can be reduced to the problen of solving the dispersion relation (4-16). We also found that the different eigensolutions are parameterized by the revolution mode number $n$, and that the eigenfunction is given by

$$
\rho_{1}(\phi, t)=e^{i n \phi-i \Omega t}
$$

with the coherent frequency $\Omega$ satisfying the dispersion relation (4-16). From Eq. (4-17), we see that the stability of this mode is determined by the imaginary part of $\Omega$; $\operatorname{Im}(\Omega)>0,=0$, or $<0$ corresponds, respectively, to the mode being urstable, stationary, or damped. We call $\operatorname{Im}(\Omega)$ the growth rate of the $n-t h$ mode, and $l / \operatorname{Im}(\Omega)$ the growth time.

\subsection{Solution of Dispersion Relation (without Landau Damping)}

Assume that there is no momentum spread in the beam (cold beam) and therefore no revolution frequency spread. This situation is described by

$$
g(\delta)=\delta(\delta)
$$

We obtain imediately from Eqs. (4-16a) and (4-18) an expression for the coherent frequency:

$$
\Omega= \pm \frac{\omega_{0}}{B_{0}} \sqrt{i \frac{\eta}{2 \pi E_{0}} e I_{a v} n z_{n}\left(n \omega_{0}+\Omega\right)} .
$$

The following conclusions can be drawn from Eq. (4-19):

(i) The cold beam is always unstable if the impedance has a resistive (real) component.

(ii) In the case where the impedance is purely reactive (purely imaginary), the beam is stable (unstable) above transition, $\eta>0$, if

the impedance is inductive (capacitive).

Below transition, $\eta<0$, conclusion ( $i i)$ above is reversed.

4.4 Solution of Dispersion Relation (with Landau Damping)

We have seen that a cold beam is quite unstable. The effect of the frequency spread is investigated here. We shall see that the frequency spread prohibits the coherent instability ${ }^{30}$ (trandau jamping) unless the current is large enough. For a given impedance and a given frequency spread, the smallest current for which the instability occurs is called the threshold current.

To find the threshold condition, we first transform Eq. (4-16b) into another form. Noting, for $\operatorname{Im}(\Omega)>0$, that

$$
\frac{1}{\Omega+\pi \omega_{0} n \delta}=-i \int_{0}^{\infty} d \tau e^{i \tau\left(\Omega+\pi \omega_{0} n \delta\right)},
$$

we have 


$$
\int_{-\infty}^{\infty} \mathrm{d} \delta \frac{g^{\prime}(\delta)}{\Omega+\pi \omega_{0} \pi \delta}=-i \int_{0}^{\infty} \mathrm{d} \tau \mathrm{e}^{i \tau \Omega} \int_{-\infty}^{\infty} \mathrm{d} \delta g^{\prime}(\delta) e^{i \tau \pi \omega_{0} \eta \delta} .
$$

Let us concentrate on the case of the beam with a Gaussian momentum distribution:

$$
g(\delta)=\frac{1}{\sqrt{2 \pi \sigma_{\delta}}} \mathrm{e}^{-\delta^{2} / 2 \sigma_{\delta}^{2}}
$$

Then we have

with

$$
\int_{-\infty}^{\infty} d \delta \frac{g^{\prime}(\delta)}{\Omega+n \omega \omega_{0} n \delta}=-\frac{1}{n \omega_{0} n \sigma_{\delta}^{2}} h_{L}\left(\frac{\Omega}{|n \eta| \omega_{0} \sigma_{\delta}}\right),
$$

$$
h_{L}(x)=\int_{0}^{\infty} d \tau \tau e^{i x t} e^{-\tau^{2} / 2},
$$

and the dispersion relation $(4-16 b)$ becomes

$$
1=-i \frac{1}{2 \pi \beta_{0}^{2} n E \sigma_{\delta}^{2}} e_{a v} \frac{z_{n}(n(\omega)+\Omega)}{n} h_{L}\left(\frac{\Omega}{|n \eta| \omega_{0} \sigma_{\delta}}\right) \text {. }
$$

Note from Eq. (1.6) that $\omega_{0} \mid n, \sigma_{\delta}$ is the r.m.s. revolution frequency spread $\sigma_{\phi}^{*}$, therefore Eq. $(4-24)$ can also be written as

$$
1=-i \frac{1}{2 \pi m B_{0}^{2} E_{0} \sigma_{\delta}^{2}} \text { eI } \frac{z_{n}\left(n w_{0}+\delta \zeta\right)}{n}-h_{L}\left(\frac{\Omega}{|n| \sigma_{\dot{\phi}}}\right) .
$$

It should be emphasized that for a Guassian beam Eq. (4-25) is equivalent to Eq. (4-16) for $\operatorname{Im}(\Omega)>0$. We use Im to indicate the imaginary part and $R e$ to indicate the real part.

In the following, we utilize Eq. (4-25) to find a sufficient condition 31,32 of the beam stability.

First we observe from Eq. (4-23) that

$$
\left\{\begin{array}{l}
h_{L}(0)=1, \\
\left|h_{L}(x)\right|<1, \text { if } \operatorname{Im}(x)>0, \text { or if } \operatorname{Im}(x)=0 \text { and } \operatorname{Re}(x) \neq 0, \\
(4-26)
\end{array}\right.
$$

It follows that

$$
1>\frac{1}{2 \pi|\eta| \beta_{0}^{2} E_{0} \sigma_{\delta}^{2}} \operatorname{eI} a \frac{\left.\Sigma_{n}(n \omega)+\Omega\right)}{n} \mid
$$


is a sufficient condition that there is no solution $\Omega$ of Eq. (4-16) with $\operatorname{Im}(\Omega)>0$, or that the beam is stable.

Exercise: Show that Eq. $(4-25)$ reduces to Eq. $(4-19)$ in the linit $\operatorname{Im}(\Omega) /\left(|n| \sigma_{\phi}^{\circ}\right) \gg 1$.

\section{BUNCKRD BEAM IONGITUDINAL MICROWAVE INSTABILITY}

It has been conjectured that the coasting beam instability eriteria apply to a bunched beam provided that the following conditions are met.

(i) The wayelenths of perturbing EM fields « bunched length.

(ii) Growth rate of the instability $>>$ synchrotron frequency. Such an instability is known as the microwave instability.

Here we take condition ( $i i$ ) above to mean that ve can set the angular synchroeron frequency $\omega_{s}=0$.

A bunched beam with momentum spread but without synchrotron focusing must filament, since particles with different momenta rotate around the ring with different revolution frequencies. We assume that the growth rate of the instability is $\gg$ the filamentation rate, so that the filamentation can be ignored.

In Section 10, we discuss the microwave instability keeping $\omega_{s}$ finite, and we find that the conclusions reached here remain valid.

\subsection{Line Density Modulation for a Bunched Beam}

We have to find the bunched beam line density modulation due to the particle displacement caused by the perturbation. The derivation is similar to that in Section 4.1, the main difference being that $\lambda\left(\phi_{0}\right)$ in Eq. (4-3) can no longer be set to $1 / 2 \pi$. Also, the cerms proportional to $\omega_{0} \eta \delta t$ in Eq. $(4-2)$ are ignored; this amounts to ignoring the filamentation.

Repeating, with the above modifications, the calculation in Section 4.1 that led to Eq. (4-9), we obtair

$$
\begin{array}{r}
\rho(\phi, t)=\lambda(\phi)+\rho^{(1)}(\phi, t), \\
\rho^{(1)}(\phi, t)=-\int d \delta g(\delta) \frac{\partial}{\partial \phi}(\zeta(\phi, \delta, t) \lambda(\phi)) .
\end{array}
$$

From

and

$$
\rho^{(1)}(\phi, t)=\sum_{n} \int_{n} d \Omega \rho_{n}(\Omega) e^{i n \phi-i \Omega t}
$$

$$
\zeta(\phi, \delta, t)=\sum_{n} \int_{n} d \Omega \zeta_{n}(\delta, \Omega) e^{i n \phi-i \Omega t}
$$


Eq. (5-2) becomes

$$
\rho_{n}(\Omega)=-i n \sum_{m=-\infty}^{\infty} \lambda_{n-m} \int d \delta g(\delta) \zeta_{m}(\delta, \Omega),
$$

where $\lambda_{n}$ is defined by

$$
\lambda(\phi)=\sum_{n=-\infty}^{\infty} \lambda_{n} e^{i n \phi}
$$

Note that Eq. (5-2) reduces to the coasting beam result $(4-9)$ if $\lambda(\phi)=1 / 2 \pi$.

\subsection{Secular Equation}

The equation of motion for a particle in the beam is Eq. (4-14). The eigenmodes of the present problem are provided by the self-consistert solutions of Eqs. $(4-14)$ and (5-2).

It can be seen frow Eq. (5-3) that the bunched structure of the unperturbed line density $\lambda(\phi)$ introduces an intrinsic coupling of the revolution nodes; the $\mathrm{m}-\mathrm{th}$ Fourier component of $\zeta$ contributes to $\rho_{n}$ within the bandwidth of $\lambda_{n-m}$. This is the key to understanding microwave instabilities.

Let us calculate $\zeta_{n}(\delta, \Omega)$ from Eq. $(4-14)$ and then substitute the result into Eq. $(5-3)$. The result is the following secular equation involving an dimensional matrix:

$$
\begin{aligned}
& \rho_{\mathrm{n}}=\operatorname{ieI}_{\mathrm{av}} \bar{\eta}_{\mathrm{m}} \sum_{\mathrm{n}=-\infty}^{\infty} \lambda_{\mathrm{m}-\mathrm{n}^{\mathrm{n}}} \mathrm{Z}_{\mathrm{n}} \int \mathrm{d} \delta \frac{\mathrm{g}(\delta)}{\left(\Omega+n \eta \omega_{\mathrm{o}} \delta\right)^{2}} \rho_{\mathrm{n}} \\
& =i e \frac{e I_{a v^{\prime}} \omega}{E_{0} \beta_{0}^{2}} \sum_{n} \frac{I}{n} \lambda_{m-n} z_{n} \int d \delta \frac{g^{\prime}(\delta)}{\Omega+n \omega_{0} \delta} \rho_{n},
\end{aligned}
$$

where we have used the abbreviation $z_{n}=z_{n}\left(n w_{0}+\Omega\right)$. These equations are generaiizations of Eq. (4-16). Note that Eqs. (5-5) and (5-6) reduce to $(4-16)$ if $\lambda(\phi)=1 / 2 \pi$ or, equivalently; if $\lambda_{\mathrm{m}-\mathrm{n}}=\delta_{\mathrm{m}, \mathrm{n}} / 2 \pi$. We explore the physical contents of the secular equation above in the next two sections by solving it for some simple impedance $z_{n}$. Let us define for later use the r.m.s. bunch length $\sigma_{\phi}$ by

$$
\sigma_{\phi}^{2}=\int_{-\infty}^{\infty} \phi^{2} \dot{d} \phi \lambda(\phi) \text {. }
$$

Then, from the property of the Fourier transform (5-4), we have

$$
\left|\lambda_{n}\right| \ll 1 \text { if }|\mathrm{n}| \sigma_{\phi} \gg 1 \text {. }
$$




\subsection{Instability Due to a High q Impedance}

Suppose the source of the ring impedance consists of a single resonance structure with its quality factor q so high that to a good approximation

$$
z_{n}=z_{n_{0}} \delta_{n, n_{0}}+z_{n_{0}}^{*} \delta_{n,-n_{0}} .
$$

Further, suppose that the wavelength corresponding to this resonance structure is much shorther than the bunch length:

$$
\sigma_{\phi}>1 / \mathrm{n}_{0}
$$

It follows from Eqs. (5-8) and (5-10) that, for a given $m$, at least one of $\lambda_{m-n_{0}}$ and $\lambda_{m+n_{0}}$ is negligible. Thus, Eq. (5-5) implies the existence of two classes of solutions: One consists of eigenvectors $\rho_{m}$ with $\left|m-n_{0}\right| s i / \sigma_{\phi}$ and the other with $\left|m+n_{0}\right| \leqslant 1 / \sigma_{\phi}$. For the first class of solutions, Eq. (5-5) becomes

$$
\rho_{\mathrm{m}}=i e I_{\mathrm{av}} \overline{\mathrm{n}} \mathrm{m} \lambda_{\mathrm{m}-\mathrm{n}_{0}} z_{\mathrm{n}_{0}} j \mathrm{~d} \delta \frac{\mathrm{g}(\delta)}{\left(\Omega+\mathrm{n}_{0} \pi \omega_{0} \delta\right)^{2}} \rho_{\mathrm{n}_{0}} .
$$

- For $m=n_{0}$, this equation becomes

$$
1=\frac{i}{2 \pi} \bar{n}_{i} \text { eI } \mathrm{n}_{0} z_{n_{0}} \int_{d} d \delta \frac{g(\delta)}{\left(\Omega+n_{0} n_{0} \delta\right)^{2}}
$$

where $\lambda_{0}=1 / 2 \pi$, which follows from Eqs. $(5-4)$ and $(4-4)$, has been used. Comparing this with $(4-16 a)$, we conclude that for a very high $q$ and high frequency impedance, the frequency shift of a bunched beam instability is identical to that of a coasting beam with the same average current.

Now let us look at the corresponding eigenvector. From Eqs. $(5-11)$ and $(5-12)$,

$$
\rho_{m}=\frac{m}{n_{0}} \lambda_{m-n_{0}} \rho_{0}
$$

In coordinate space, upon using Eqs. (5-13), (4-10), and (5-4), one obtains

$$
\rho^{(1)}(\phi, t)=-i \frac{1}{n_{0}} \frac{\partial}{\partial \phi}\left(\lambda(\phi) e^{i n_{0} \phi-i \Omega t}\right) \rho_{n_{0}} .
$$




$$
\int d \delta g(\delta) \zeta(\phi, \delta, t)=-i \frac{\rho_{n_{0}}}{n_{0}} e^{i n_{0} \phi-i \Omega_{t}} .
$$

The meaning of Eq. $(5-15)$ is that the particles, being excited by the high $q$ impedance, are executing harmonic motions just like the particles in a coasting beam. Note that $(5-15)$ does not vanish outside the bunch. However, in the expression $(5-14)$ for the line density this harmonic motion is modulatd by $\lambda(\phi)$ so that the beam stays bunched.

Equation (5-14) is a complex expression despite the fact that the line density has to be real; where is the complex conjugate of $(5-14) ?$

Exercise: Prove that the other class of solutions with

$$
\begin{aligned}
& \left|m+n_{0}\right| s 1 / \sigma_{\phi} \text { contribute a part to } \rho^{(1)}(\phi, t) \text { which } \\
& \text { is the complex conjugate of }(5-14) \text {. Hint: } \\
& z_{n}^{*}\left(n \omega_{0}+\Omega\right)=z_{n}\left(-n \omega_{0}-\Omega^{*}\right) \text {. }
\end{aligned}
$$

Exercise: Suppose

$$
z_{n}=z_{n_{0}} \delta_{n, n_{0}}+z_{n_{0}}^{*} \delta_{n,-n_{0}}+z_{m_{0}} \delta_{n, m_{0}}+z_{n_{0}}^{*} \delta, n,-m_{0}
$$

with

$$
\begin{aligned}
& \text { (i) } \mathrm{z}_{\mathrm{n}_{0}}=\mathrm{z}_{\mathrm{u}_{0}}, \\
& \text { (ii) } \mathrm{n}_{O_{\phi}} \gg 1, \mathrm{~m}_{0} \sigma_{\phi} \gg 1 \text {, and }\left|\mathrm{n}_{0}-\mathrm{m}_{0}\right| \sigma_{\phi} \ll 1 \text {. } \\
& \text { Show that one obtains approximately a dispersion re- } \\
& \text { lation identical to }(5-12) \text { except that the } \mathrm{I}_{\mathrm{av}} \text { in } \\
& \text { (5-12) is replaced by } 2 \mathrm{I}_{\mathrm{av}} \text {. }
\end{aligned}
$$

\subsection{High Frequency Instability Due to a Broad Band Impedance}

We now consider the high frequency instability due to a wakefield whose range is short compared to the bunch length $\sigma_{\phi}$. To be more specific, we assume that there is an integer $n_{0}$ such that

$$
\text { (i) } z_{n}=z_{n_{0}} \text { if } \quad\left|n-n_{0}\right| \leq \Delta
$$

where $\Delta$ is of the order of the inverse range of the wakefield;

$$
\text { (ii) } n_{0} \gg \Delta \gg 1 / \sigma_{\phi} \text {. }
$$

Let us find the approximate solutions of Eq. (5-6) for which $\rho_{\mathrm{m}}$ is negligibly small when $\left|n-n_{0}\right|>\Delta$. Then, from the conditions (i) and (ii) above, the secular equation $(5-6)$ can be approximated by 


$$
\rho_{m}=i \frac{e I_{a v} \omega_{0}}{E_{0} \beta_{0}^{2}} z_{n_{0}} \int d \delta \frac{g^{\prime}(\delta)}{\Omega+n_{0} \delta^{\delta} \omega_{0}} \sum_{n=n_{0}-\Delta}^{n_{0}+\Delta} \lambda_{m-n} \rho_{n} .
$$

Denote by $k$ the eigenvalue of the matrix $\lambda_{m-n}$ :

$$
K \rho_{m}=2 \pi \sum_{n=n_{0}-\Delta}^{n_{0}+\Delta} \lambda_{m-n} \rho_{n} .
$$

Then the coherent frequency shift $\Omega$ is determined by

$$
1=i \kappa \frac{e I a w_{0}^{\omega}}{2 \pi E_{0} \beta_{0}^{2}} z_{n_{0}} \cdot d \delta \frac{g^{\prime}(\delta)}{\Omega+n_{0} \omega_{0} \delta} .
$$

It remains for us to find $k$ by solving Eq. (5-17). Since $\lambda_{\mathrm{m}-n}$ is sharply peaked about $m=n$, the peak width being of orcier $1 / \sigma_{\phi}^{\mathrm{m}-n}<<$ $\Delta$, we expect that the eigenvalues do not depend strongly on the cutoff value $\Delta$. Therefore they should be closely approximated by

$$
k \rho_{m}=2 \pi \sum_{n=-\infty}^{\infty} \lambda_{m-n} \rho_{n} .
$$

The eigenfunctions of Eq. $(5-19)$ are

$$
\rho_{n}(\Phi)=e^{-i \Phi_{n}}
$$

and the corresponding eigenvalues are

$$
\kappa(\Phi)=2 \pi \sum_{n=-\infty}^{\infty} \lambda_{n} e^{i \Phi n}=2 \pi \lambda(\Phi)
$$

where $\Phi, 0 \leq \Phi<2 \pi$, is a parameter which 1 abels the different eigensolutions.

Note that Eq. $(5-18)$ is the same as the coasting beam dispertsion relation (4-16b) except that $I_{a v}$ in $(4-16 b)$ is replaced by $K I_{a v}$ in (5-18). The effective current for the mode $\Phi$ is

$$
\begin{aligned}
I_{e f f}(\Phi) & =k(\Phi) I_{a v}=2 \pi \lambda(\Phi) \frac{e N \omega_{o}}{2 \pi} \\
& =e N \omega_{o} \lambda(\Phi) .
\end{aligned}
$$

This is the local current at position $\phi=\Phi$ in the bean. The most severe instability happens when $I_{\text {eff }}$ is maximal, i.e., at $\Phi=0$, $I_{\text {ef }}(\Phi=0)=I_{\text {peak }}$. 
To gain some insight into the nature of the perturbed line density, let us make the following approximation to the eigenvectors of $(5-17)$ :

$$
\begin{aligned}
& \rho_{n}(\Phi)=e^{-i \Phi n} \quad \text { for } \quad\left|n-n_{0}\right| \leq \Delta \\
& =0 \text { for }\left|n-n_{0}\right|>\Delta \text {. }
\end{aligned}
$$

The perturbation to the charge density is

$$
\begin{aligned}
& \left.\rho^{(1)}(\phi, t)=\sum_{n=\Sigma_{0}-\Delta}^{n_{Q}+\Delta} \quad-i i \psi-\Phi\right)-i \Omega t \\
& =e^{i n_{0}(\phi-\phi)} \frac{\sin \left(\left(\Delta+\frac{1}{2}\right)(\phi-\phi)\right)}{\sin ((\phi-\phi) / 2)} e^{i \Omega t} .
\end{aligned}
$$

For large $\Delta, \rho^{(1)}(\phi, t)$ is sharply peaked about $\phi=\Phi$, and the peak width is of order $1 / \Delta \sim$ range of wakefield. The detailed structure within the peak depends on the detailed short distance behavior of the wakefield, which has been ignored in making the approximation of (i) and hence is outside our discussion.

A computer calculation 19 to check the approximation of Eq. $(5-17)$ by $(5-19)$ showed the error to be $<10 \%$ when $\sigma_{\phi} \Delta=3$ and $<5 \%$ when $\sigma_{\phi} \Delta=4$. The error $\rightarrow 0$ as $\sigma_{\phi} \Delta \rightarrow \infty$.

We have demonstrated here that, under the assumtions (i), (ii), and vanishing synchrotron frequency, the perturbation to the line density of an eigenmode is localized azimuthally around a point on the bunch, and the corrus jonding coherent frequency shift $\Omega$ is determined by the coasting beam dispersion relation with the $I_{a v}$ replaced by the local current at the position of the perturbation.

It is not surprising that the width of the eigenfunction of the microwave instability is of the order of the wakelength. As stated at the end of Section 3.2, the wakelength is the coherent length of the pulse of the electric field induced by the current. When the electric field reinforces the beam oscillation, the phase of the beam oscillation cannot maintain the coherence (correlation) outside this range.

Messerschmid and Month 10 first studied within the Vlasov formalism the microwave instability with a philosophy similar to ours. However, they based their analysis on the following ansatz for the eigenfunction: $\rho(1)(\phi)=e^{i n_{0} \phi} \lambda(\phi)$, which for high frequency is basically the same as our Eq. (5-14); therefore, their work is appropriate only for the case of a very narrow band impedance.

\section{ROBINSON INSTABILITY}

We have thus far ignored the effect that synchrotron motion of the particle in a bunched beam may have on coherent instability. This effect will be included in the rest of Part $I$. Let us start with the Robinson instability, since it is the simplest and also is the prototype of all the synchrotron modes. 
We consider here the case of a rigid point-like bunch of total charge Ne executing synchrotron motion in the $\mathrm{rf}$ bucket as well as rotating around the ring; the average current $I_{\text {av }}=e N \omega_{0} / 2 \pi$. The bunch may not have the synchronous phase because of the beam-induced longitudinal electric field. We also assume that the rf cavity is the only source of the beam impedance.

We shall generalize later the discussion of this section to more complicated situations. In Section 7.1 .1 , we treat the longitudinal force induced by a bunch with a finite area, and the result of that section will be used in Section 8 to discuss the generalized Robinson instability, or synchrotron modes.

Denote by $\phi(t)$ the bunch position relative to the synchronous position; then the azimuthal position of the bunch relative to the ring can be written as

$$
\theta(t)=\omega_{0} t+\phi(t)
$$

We assume that the rf cavity is located at $\theta=0$. Also, let $\mathrm{pT}_{0}+\mathrm{t}_{\mathrm{p}}$ be the time the bunch passes the cavity on its $p^{-t h}$ revolution around the ring, and $\phi_{p}$ be the value of $\phi$ at that instant, $\phi_{p}=\phi\left(p T_{0}+t_{p}\right)$. Then,

$$
2 \pi_{p}=\omega_{0}\left(p T_{0}+t_{p}\right)+\phi_{p},
$$

or

$$
\phi_{p}=-w_{0} t_{p}
$$

We can now write the equations of motion of the particle in the ? bunch as follows:

$$
\left\{\begin{array}{l}
\phi_{k}-\phi_{k-1}=-\frac{2 \pi m}{B_{0}^{2} \varepsilon_{k},} \\
\varepsilon_{k+1}-\varepsilon_{k}=\frac{e}{E_{o}}\left(v_{g}\left(k T_{o}+t_{k}\right)-v_{E}-v_{\gamma}+v_{c}\left(k T_{o}+t_{k}\right)\right),
\end{array}\right.
$$

where $\varepsilon_{k}$ is the value of $\varepsilon$ just before the bunch crosses the cavity on its $k$-th turn, and

$$
\begin{aligned}
& \nabla_{g}(t)=\text { rf voltage produced by the source current (generator } \\
& \text { current; of the rf system, } \\
& \nabla_{c}(t)=\begin{array}{l}
\text { voltage across the } \mathrm{rf} \text { cavity induced by the beam } \\
\text { current, }
\end{array} \\
& \begin{aligned}
& e V_{E}= \text { energy gain per turn of the synchronous particle due to } \\
& \text { acceleration, }
\end{aligned} \\
& e V_{Y}=\text { energy loss of the particle per } \cdots \cdots n \text { due to synchroton }
\end{aligned}
$$


Note that $\mathrm{eV}_{\gamma}$ is taken to be independent of $\varepsilon$, this amounts to ignoring the radiation damping of the longitudinal beam emittance.

We take the generator voltage to be sinusoidal:

$$
V_{g}(t)=\hat{v}_{g} \sin \left(h \omega_{o} t+\phi_{g}\right),
$$

where $\phi_{g}$ is the synchronous phase of $V_{g}$. Using this equation and Eq. $(6-2)$, we have

$$
v_{g}\left(k T_{0}+t_{k}\right)=\hat{v}_{g} \sin \left(-h \phi_{k}+\phi_{g}\right) \text {. }
$$

Next, we discuss $V_{c}(t)$. Denote by $I_{c}(t)$ the beam current at the position of the rf cavity, and define its Fourier component $I_{c}(\omega)$ by

$$
I_{c}(t)=\int_{-\infty}^{\infty} d \omega f_{c}(\omega) e^{-i \omega t}
$$

The voltage across the cavity is related to the beam current by

$$
\nabla_{c}(t)=-\int_{-\infty}^{\infty} d \omega f_{c}(\omega) z(\omega) e^{-i \omega t}
$$

where $z(\omega)$ is the longitudinal impedance of the cavity, and the minus sign in this equation reflects the fact that $V_{c}$ is generated by the image current of the beam, which equals in magnitude but is opposite in direction to the beam current. The impedance $Z(\omega)$ here is a special case of $Z_{n}(\omega)$ discussed in Section 3.2. We have just seen that the impedance from a localized source is independent of the revolution mode number $n$.

Exercise: The equation (6-7) incroduces the cavity

impedance in a way different from that of

Section 3.2. Treat the cavity impedance using the method of Section 3.2, and calculate the corresponding energy change of the particle per turn due to the beam-induced electric field. Show that the energy change so calculated agrees with Eq. $(6-10)$ up to the first order in $\Omega^{\prime} \omega_{0}$.

For the model at hand, the current

$$
I_{c}(t)=e N \sum_{p=-\infty}^{\infty} \delta\left(t-p T_{0}-t_{p}\right),
$$

and its Fourier component 


$$
f_{c}(\omega)=\frac{e N}{2 \pi} \sum_{p=-\infty}^{\infty} e^{i \omega\left(p T_{o}+t_{p}\right)} .
$$

Substitution of Eq. (6-8) into (6-7) gives

$$
\nabla_{c}\left(k T_{0}+t_{k}\right)=-\frac{e N}{2 \pi} \sum_{p=-\infty}^{\infty} \int_{-\infty}^{\infty} d \omega z(\omega) e^{i \omega\left((p-k) T_{0}+t_{p}-t_{k}\right)} .
$$

We are now ready to solve Eqs. (6-3) and (6-4). First, note that if the bunch moves along the synchronous orbit, then $\phi_{p}=0, \varepsilon_{p}=0$ for all p. Therefore, from Eqs. $(6-4),(6-6),(6-10)$ and $(6-2)$,

$$
\begin{aligned}
\hat{v}_{g} \sin \phi_{g} & =v_{E}+v_{Y}+\frac{e N}{2 \pi} \sum_{p=-\infty}^{\infty} \int_{-\infty}^{\infty} d \omega z(\omega) e^{i \omega p T_{0}} \\
& =v_{E}+v_{Y}+I_{a v} \sum_{n=-\infty}^{\infty} R\left(n \omega_{0}\right),
\end{aligned}
$$

where $(\omega)$ is the resistive part of $Z(\omega)$. In obtaining Eq. (6-11), the Poisson sum rule

$$
\sum_{p=-\infty}^{\infty} e^{i p x}=2 \pi \sum_{n=-\infty}^{\infty} \delta(x-2 \pi n)
$$

and the causality condition (3-17) have been used. Equation $(6-11)$ is a restatement of the energy conservation law. It states that the rf system provides energy for acceleration of the particles and for compensating the radiation and parasitic energy losses. Let us combine Eqs. $(6-3)$ and $(6-4)$ to obtain

$\phi_{k+1}-2 \phi_{k}+\phi_{k-1}=\frac{2 \pi n_{1}}{\beta_{0}^{2} E_{0}}\left(\hat{v}_{g} \sin \left(h \phi_{k}-\phi_{g}\right)+v_{E}+v_{\gamma}-v_{c}\left(k T_{o}+t_{k}\right)\right)$.

We shall solve this equation to the first order in $\phi_{k}$.

To this order, the equation is

$$
\begin{aligned}
\phi_{k+1}- & 2 \phi_{k}+\phi_{k-1}=\frac{2 \pi e \eta}{\beta_{o}^{2} E_{o}} \\
& \times\left(h \phi_{k} \hat{v}_{g} \cos \phi_{g}-i \frac{e N}{2 \pi \omega_{o}} \sum_{p}\left(\phi_{p}-\phi_{k}\right) \int \omega d \omega z(\omega) e^{i \omega(p-k) T_{0}}\right),
\end{aligned}
$$

where Eq. (6-11) has been used. 
To find the coherent frequency $\Omega$ of the Robinson mode, we look for the solution of Eq. $(6-13)$ of the form

$$
\phi_{p}=\bar{\phi} e^{-i \Omega_{p} T_{0}}+\bar{\phi}^{*} e^{i \Omega^{*} p T_{0}},
$$

where $\bar{\phi}$ is a constant. Substituting Eq. $(6-14)$ into (6-13), we finally obtain

$$
\begin{aligned}
\Omega^{2}= & \frac{e \bar{n}}{2 \pi}\left(-\mathrm{h} \hat{\nabla}_{\mathrm{g}} \cos \phi_{\mathrm{g}}+I_{\mathrm{av}} \sum_{\mathrm{n}=-\infty}^{\infty} \mathrm{nX}\left(\mathrm{n} \omega_{0}\right) .\right. \\
& \left.+i I_{\mathrm{av}} \sum_{\mathrm{n}=-\infty}^{\infty}\left(\pi+\frac{\Omega}{\omega_{0}}\right) z\left(n \omega_{0}+\Omega\right)\right),
\end{aligned}
$$

and

$$
\begin{aligned}
\left(-\Omega^{*}\right)^{2}= & \frac{e \bar{\eta}}{2 \pi}\left(-h \hat{V}_{g} \cos \phi_{g}+I_{a v} \sum_{n=-\infty}^{\infty} n X\left(n \omega_{0}\right)\right. \\
& \left.+i I_{a v} \sum_{n=-\infty}^{\infty}\left(n-\frac{\Omega^{*}}{\omega_{0}}\right) Z\left(n \omega_{0}-\Omega^{*}\right)\right),
\end{aligned}
$$

where $X(\omega)$ is the reactive (imaginary) part of $Z(\omega)$. These two equations determine the coherent frequencies $\Omega$ and $-\Omega^{*}$. We note from ?q. (3-17) that these equations are just the complex conjugate of ea:h other, and from Eq. (6-14) that if $\Omega$ corresponds to stability or instability, so does $\Omega^{*}$.

The angular synchrotron frequencies $\omega_{s o}$ and $\omega_{s}$ are defined by

$$
\omega_{s o}^{2}=-\frac{1}{2 \pi} \bar{\eta}_{\mathrm{heV}} \hat{\mathrm{g}}_{\mathrm{g}} \cos \phi_{\mathrm{g}},
$$

and

$$
\omega_{s}^{2}=\omega_{s o}^{2}+\frac{1}{2 \pi} \bar{\eta} \text { eI } \sum_{a v}^{\infty} n x\left(n \omega_{o}\right) .
$$

$\omega_{\text {so }}$ is the angular synchrotron frequency at zero current, and $\omega_{s}$, which includes the effect of beam loading in the absence of coherent effect, (Eq. $(6-18)$ is independent of $\Omega$ ), is the actual incoherent angular synchrotron frequency for longitudinal phase focusing.

The equation $(6-15)$ is quite complicated. However, if the first term on the right-hand side of the equation is much greater than the terms involving the impedance, the equation can be solved perturbatively. To the first order in the impedance, the solution is 


$$
\begin{aligned}
\Omega= & w_{s o}+\frac{e \bar{n}}{2 \pi} I_{a v} \sum_{n=-\infty}^{\infty}\left\{n \times\left(n w_{0}\right)-\left(n+\frac{w_{s o}}{\omega_{0}}\right) \times\left(n w_{0}+w_{s o}\right)\right\} \\
& +i \frac{e \bar{n}}{2 \pi} I_{a v} \sum_{n=-\infty}^{\infty}\left(n+\frac{\omega_{s o}}{\omega_{0}}\right) \mathscr{R}\left(n w_{0}+w_{s o}\right) .
\end{aligned}
$$

The stability of the Robinson mode is determined by the sign of the last term of this equation.

A way of avoiding the Robinson instability, Robinson damping, will be discussed in Section 8 .

\section{VLASOV EQUATION FOR SINGLE BUNCH LONGITUDINAL COHERENT INSTABILITY}

In Section 7 we studied the coherent instability of a rigid point-like beam. We extend the method here so that we can handle the case of a finite sized beam with possible beam shape distortions. The method best suited to this purpose is that of vlasov.

The phase space density function of a canonical system satisfies the Liouville equation, 33 which involves the force field on the particle as a coefficient. The force field is related to the electromagnetic fields, which satisfy the Maxwell equations with source terms dependent on phase space density. Vlasov's method consists of finding self-consistent solutions of the Liouville equation (called the vlasov equation in this context) and the Maxwell equations.

In our treatment of the longitudinal coherent instability, the Maxwell equations are represented by (3-14).

This section is devoted to formulation of the vlasov equation

for longitudinal coherent instabilities and development of a method 15-19

for solving the equation in the linear approximation. Landau damping due to the synchrotron frequency spread will be ignored. 34

The vlasov equation for some specific cases will be solved in the remainder of Part $I$.

\subsection{Equations of Motion}

The equations of motion are

$$
\dot{\phi}=-\frac{n \omega_{0}}{\beta_{0}^{2}} \varepsilon,
$$

$$
\dot{\varepsilon}=-\frac{e \omega_{0}}{2 \pi E_{0}}\left(\hat{v}_{g} \sin \left(h \phi-\phi_{g}\right)+v_{Y}+v_{E}\right)+\frac{e R}{E_{0}}\left(\omega_{0}+\dot{\phi}\right) \mathscr{E}\left(\omega_{0}+\phi, t\right),
$$

where the quantities within the brackets have been defined in the peeceding secition. 


\subsubsection{Beam-induced force}

The E⿱ term in Eq. (7-2) describes the effect of the electric field on the beam particle, and the electric field itself is in turn induced by the beam. Here we express this term in terms of the particle line density; we include the small effect caused by the synchrotron motion of the particles (the $\phi$ term).

The contribution of the j-th particle to the beam current is

$$
\begin{aligned}
I_{j}(\theta, t) & =e\left(\omega_{0}+\dot{\phi}_{j}(t)\right) \delta_{p}\left(\phi-\phi_{j}(t)\right) \\
& =e \int d \dot{\phi}\left(\omega_{0}+\dot{\phi}\right) \delta_{p}\left(\phi-\phi_{j}\right) \delta\left(\dot{\phi}-\dot{\phi}_{j}\right),
\end{aligned}
$$

where $\delta_{p}$ is the periodic delta function with period $2 \pi$; hence the total beam current is

$$
I(\theta, t)=e \sum_{j=1}^{N} \int d \dot{\phi}\left(\omega_{0}+\dot{\phi}\right) \delta_{p}\left(\phi-\phi_{j}\right) \delta\left(\dot{\phi}-\dot{\phi}_{j}\right) .
$$

$\phi$ is small compared to $\omega_{0}$. However, instead of dropping the term proportional to $\phi$, we average Eq. (7-4) over a revolution period $T_{0}=2 \pi / \omega_{0}$ under the assumption that $\phi_{j}(t)$ is constant in this period; the variation of $\phi_{j}$ from one revolution period to another will be taken into account. The reason for this maneuver is that, while $\phi_{j}$ changes very little within a revolution period $\left(\phi_{j} / \omega_{0} \ll 1\right)$, the change in $\phi$ accumulated over an interval comparable to a synchrotron period may affect the coherent instability. Th ${ }_{-3}$ is indeed the case if there is a component of impedance that changes appreciably within the range of frequency of the order of the synchrotron frequency. The rf cavity itself is generally a source of such an impedance.

The Fourier component of the current is, from Eq. (7-4),

$$
\begin{aligned}
& I_{n}\left(n \omega_{0}+\Omega\right)=\frac{e}{4 \pi^{2}} \sum_{j=1}^{N} \sum_{p=-\infty}^{\infty} \int_{-\infty}^{\infty} d \dot{\phi} \int_{0}^{2 \pi} d \theta \int_{-\infty}^{\infty} d t\left(\omega_{0}+\dot{\phi}_{j}\right) \\
& \times \delta\left(\theta-\omega_{0} t-\phi_{j}(t)+2 \pi_{p}\right) \delta\left(\dot{\phi}-\dot{\phi}_{j}\right) e^{-i n \phi_{j}(t)+i \Omega t} .
\end{aligned}
$$

Let us define a function $\tau_{j}(\theta)$ implicitly by

$$
\theta-\omega_{0} \tau_{j}(\theta)-\phi_{j}\left\{\tau_{j}(\theta)\right\}=0, \quad-\infty<\theta<\infty .
$$

The function $\tau_{j}(\theta+2 \pi p)$ with integer $p$ and $0 \leq \theta<2 \pi$ gives us the arrival time of particle $j$ at azimuth $\theta$ during the $p$-th revolution. Note that

$$
\left(\omega_{o}+\dot{\phi}_{j}(t)\right) \delta\left(\theta-\omega_{o} t-\phi_{j}(t)+2 \pi_{p}\right)=\delta\left(t-\tau_{j}\left(\theta+2 \pi_{p}\right)\right) .
$$


Substituting this expression, into Eq. (7-5), and then performing the integrations over $t$ and $\dot{\phi}$, gives

$I_{n}\left(n \omega_{0}+\Omega\right)=\frac{e}{4 \pi^{2}} \sum_{j=1}^{N} \sum_{p=-\infty}^{\infty} \int_{0}^{2 \pi} d \theta e^{-i n \phi_{j}}\left(\tau_{j}(\theta+2 \pi p)\right)+i \Omega \tau_{j}(\theta+2 \pi p)$,

which, with use of Eq. $(7-6)$; can be written as

$$
\begin{aligned}
& I_{L}\left(n \omega_{0}+\Omega\right)=\frac{e}{4 \pi^{2}} \sum_{j} \sum \int_{0}^{2 \pi} d \theta \\
& \times e^{-i\left(n+\frac{\Omega}{\omega_{0}}\right) \phi_{j}\left\{\tau_{j}(\theta+2 \pi p)\right\}+i \Omega p T_{0}+i \frac{\Omega}{\omega_{0}} \theta} .
\end{aligned}
$$

We now ignore the variation of $\phi_{j}(t)$ within a revolution period; i.e., we set

$$
\phi_{j}\left\{\tau_{j}(\theta+2 \pi p)\right\}=\phi_{j}\left\{\tau_{j}(2 \pi p)\right\} \text {. }
$$

The integration in Eq. (7-7) can then be perforned. We obtain

$$
I_{n}\left(n \omega_{0}+\Omega\right)=\frac{e}{2 \pi} \Gamma\left(\frac{\Omega}{\omega_{0}}\right) \sum_{j} \sum_{p} e^{-i\left(n+\frac{\Omega}{\omega_{0}}\right) \phi_{j}\left\{\tau_{j}(2 \pi p)\right\}+i \Omega p T_{0}}
$$

with

$$
\Gamma(x)=-i \frac{1}{2 \pi x}\left(e^{i 2 \pi x}-1\right)
$$

We are interested only in the region of $\Omega$ where $\Omega T_{0} \ll 1$. Therefore, we set $\mathrm{pT}_{0} \rightarrow t, \phi_{j}\left(\tau_{j}(2 \pi p)\right) \rightarrow \phi_{j}(t)$, and change the summation over $p$ by an integral,

$$
\sum_{p=-\infty}^{\infty} \rightarrow \frac{1}{T_{0}} \int_{-\infty}^{\infty} d t
$$

The result is

$$
I_{n}\left(n \omega_{0}+\Omega\right)=\frac{e \omega_{0}}{4 \pi^{2}} \Gamma\left(\frac{\Omega}{\omega_{0}}\right) \sum_{j=1}^{N} \int_{-\infty}^{\infty} d t e^{-i\left(n+\frac{\Omega}{\omega_{0}}\right) \phi_{j}(t)+i \Omega t}
$$

Equation (7-9) can be expressed in terms of the line density. The line density corresponding to $(7-3)$ is

$$
\rho(\phi, t)=\frac{1}{N} \sum_{j=1}^{N} \delta_{p}\left(\phi-\phi_{j}(t)\right) \text {. }
$$

Define 


$$
\tilde{\rho}_{n}+\Omega / \omega_{c}(\Omega)=\frac{1}{4 \pi^{2}} \int_{0}^{2 \pi} \mathrm{d} \phi \int_{-\infty}^{\infty} \mathrm{d} \mathrm{c} \mathrm{e}^{-i\left(n+\Omega / \omega_{0}\right) \phi+i \Omega} \mathrm{t}_{p}(\phi, t) .
$$

Then, the above two equations give

$$
\stackrel{\rho}{\rho}_{n}+\Omega /(1)=\frac{1}{4 \pi^{2} N} \sum_{j=1}^{N} \int_{-\infty}^{\infty} d t e^{-i\left(n+\Omega / \omega_{0}\right) \phi_{j}+i \Omega t} .
$$

We Einally obtain frca Eqs. $(7-9)$ and $(7-10)$

$$
I_{n}\left(n \omega_{0}+\Omega\right)=2 \pi \Gamma\left(\frac{\Omega}{\omega_{0}}\right) I_{a v} \tilde{\rho}_{n+\Omega / \omega_{0}} .
$$

This is the form of the current source we shall use.

We now perform a similar averaging procedure for the term $\left(\omega_{0}+\phi\right) \mathscr{E}\left(\omega_{0}+\phi, t\right)$ in Eq. $(7-2)$. First define

$$
\mathscr{E}(\theta, t)=\sum_{n} \int_{\mathrm{d}} \mathrm{d} \omega \mathscr{E}_{n}(\omega) \mathrm{e}^{\mathrm{in} \theta-\mathrm{i} \omega t} .
$$

Consider a particle with its position $\phi(t)$ moving in the electric field above. The rate of the energy gain of the particle from $\mathscr{E}$ is

$$
\begin{aligned}
\dot{E}(t) & =e R\left(\omega_{0}+\dot{\phi}(t)\right) \mathscr{E}\left(\omega_{0} t+\phi(t), t\right) \\
& =e R\left(\omega_{0}+\dot{\phi}(t)\right) \sum_{n} \int_{d} d \mathscr{E}_{n}\left(n \omega_{0}+\Omega\right) e^{i n \phi(t)-\Omega t} .
\end{aligned}
$$

Denote by $\tau(2 \pi p+\theta), 0 \leq \theta<2 \pi$, the time of arrival of the particle at the azimuth $\theta$ during its $p$-th revolution. It is determined implicitly by

$$
\theta-\omega_{0} \tau(\theta)-\phi\{\tau(\theta)\}=0, \quad-\infty<\theta<\infty .
$$

Equation (7-13) can be written as

$$
\begin{aligned}
& \dot{E}(t)=e R\left(\omega_{0}+\dot{\phi}(t)\right) \sum_{n} \sum_{p=-\infty}^{\infty} \int d \Omega \mathscr{E}_{n}(n \omega+\Omega):_{0}^{2 \pi} d \theta \\
& x \delta\left(\theta-\omega_{0} t-\phi(t)+2 \pi p\right) e^{i n \phi(t)-i \Omega t} \\
& =\operatorname{eR} \sum_{n} \sum_{p}\left\lceil d \Omega \mathscr{E}_{n}\left(n \omega_{0}+\Omega\right) \int_{0}^{2 \pi} d \theta \delta(t-\tau(2 \pi p+\theta)) e^{i n \phi(t)-i \Omega t} .\right.
\end{aligned}
$$


The p-term in the sumation contributes only during the $\mathrm{p}$-th revolution. Hence, if we integrate this term over $t$ and then divide the result by $T_{0}$, we obtain the average rate of the energy gain in the same revolution. It is

$$
\begin{aligned}
\langle\dot{E}\rangle= & \left.\frac{e \omega_{0}^{R}}{2 \pi} \sum_{n} \int d_{\omega i} E_{n}(n \omega)_{0}+\Omega\right) \int_{0}^{2 \pi} d \theta e^{i n \phi\{\tau(2 \pi p+\theta)\}-i \Omega \tau(2 \pi p+\theta)} \\
= & \frac{e \omega}{2 \pi} \sum_{n} \int d \Omega \mathscr{E}_{n}\left(n \omega_{0}+\Omega\right) \int_{0}^{2 \pi} d \theta \\
& \times e^{i\left(n+\frac{\Omega}{\omega_{0}}\right) \phi\{\tau(2 \pi p+\theta)\}-i \Omega p T_{0}-i \frac{\Omega}{\omega_{0}} .} .
\end{aligned}
$$

Again we set

$$
\phi\{\tau(2 \pi p+\theta)\} \rightarrow \phi\{\tau(2 \pi p)\},
$$

and Eq. $(7-16)$ becomes

$\dot{\vec{E}}\rangle=e_{0} \omega_{0} \sum_{n} \int d \Omega \mathscr{E}_{n}\left(n \omega_{0}+\Omega\right) \Gamma^{*}\left(\frac{\Omega}{\omega_{0}}\right) e^{i\left(n+\frac{\Omega}{\omega_{0}}\right) \phi\{\tau(2 \pi p)\}-i \Omega p T_{0}}$.

We now set $\mathrm{pT}_{\mathrm{o}} \rightarrow t, \phi\{\tau(2 \pi p)\} \rightarrow \phi(t)$, and drop the average sign $<$ from $\langle\mathrm{E}\rangle$. We obtain

$$
\dot{\mathrm{E}}=\omega_{0} R \sum_{\mathrm{n}} \int \mathrm{d} \Omega \mathscr{E}_{\mathrm{n}}\left(n \omega_{0}+\Omega\right) \Gamma^{*}\left(\frac{\Omega}{\omega_{\Omega}}\right) \mathrm{e}^{i\left(n+\frac{\Omega}{\omega_{0}}\right) \phi(t)-i \Omega t}
$$

Or ,

$$
\begin{gathered}
\left.\left(\omega_{0}+\dot{\phi}\right) \mathscr{E}\left(\omega_{0} t+\phi(t), t\right)=\omega_{0} \sum_{n} \int d \Omega \mathscr{E}_{n}\left(n \omega_{0}+\Omega\right) \Gamma^{*}\left(\Omega / \omega_{0}\right)\right) \\
\times e^{i\left(n \frac{\Omega}{\omega_{0}}\right) \phi(t)-i \Omega t},
\end{gathered}
$$

where $\Gamma$ is given by Eq. (7-8).

From Eqs. $(7-12)$ and $(3-14 c)$,

$$
\mathscr{E}_{n}\left(n \omega_{0}+\Omega\right)=-\frac{1}{2 \pi} I_{n}\left(n \omega_{0}+\Omega\right) Z_{n}\left(n \omega_{0}+\Omega\right)
$$

The function $\left(\omega_{0}+\dot{\phi}\right) \mathscr{E}$ can now be expressed in terms of the line density by combining Eqs. $(7-19),(7-20)$, and $(7-11)$. Noting that 


$$
\Gamma\left(\frac{\Omega}{\omega_{0}}\right) \Gamma^{*}\left(\frac{\Omega}{\omega_{0}}\right)=1+0\left(\frac{\Omega^{2}}{\omega_{0}^{2}}\right)
$$

and ignoring terms of $0\left(\Omega^{2} / \omega_{0}^{2}\right)$, we obtain

$$
\begin{aligned}
\left(\omega_{0}+\dot{\phi}\right) \mathscr{E}\left(\omega_{0} t+\phi, t\right)= & -\frac{1}{R} \omega_{0} I_{a v} \sum_{n} \int d \Omega \\
& \times \stackrel{\rho}{n}_{n+\Omega / \omega_{0}}(\Omega) z_{n}\left(n \omega_{0}+\Omega\right) e^{i\left(n+\Omega / \omega_{0}\right) \phi-i \Omega t}
\end{aligned}
$$

We have thus succeeded in finding the expression for the beaminduced force (up to first order in $\Omega / \omega_{0}$ ) in terms of the line density.

\subsubsection{Linear approximation of equation of motion} motion,

First we combine Eqs. (7-1) and (7-2) into a single equation of

$$
\begin{aligned}
\ddot{\phi}= & \frac{e \bar{\eta}}{2 \pi}\left(\left\{\hat{v}_{g} \sin \left(h \phi-\phi_{g}\right)+\nabla_{Y}+\nabla_{E}\right\}\right. \\
& \left.-2 \pi R\left(1+\frac{\dot{\phi}}{\omega_{0}}\right) \mathscr{E}\left(\omega_{0} t+\phi, t\right)\right) .
\end{aligned}
$$

Recall that $\bar{n}=\pi \omega_{0}^{2} /\left(\beta_{0}{ }^{2} E_{o}\right)$.

Before using Eq. $(7-21)$ in (7-22), we split the line density:

$$
\rho(\phi, t)=\lambda(\phi)+\rho^{(1)}(\phi, t),
$$

where $\lambda(\phi)$ is the line density in the absence of coherent motion induced by the self-force $\mathscr{E}$, and $\rho(1)(\phi, t)$ describes the coherent motion. Define, for any conplex number $\alpha$,

and

$$
\begin{aligned}
\lambda_{\alpha} & =\frac{1}{2 \pi} \int_{0}^{2 \pi} \mathrm{d} \phi \mathrm{e}^{-i \alpha \phi} \lambda(\phi), \\
\rho_{\alpha}(\Omega) & =\frac{1}{4 \pi^{2}} \int_{0}^{2 \pi} \mathrm{d} \phi \int_{-\infty}^{\infty} \mathrm{dt \rho}(1) \mathrm{e}^{-i \alpha \phi+i \Omega \mathrm{t}},
\end{aligned}
$$

$$
\tilde{\rho}_{\alpha}(\Omega)=\frac{1}{4 \pi^{2}} r_{0}^{2 \pi} d \phi \Gamma_{-\infty}^{\infty} d t \rho(\phi, t) e^{-i \alpha \phi+i \Omega t} .
$$

These Fourier components are related, from Eq. (7-23), by 


$$
\hat{\rho}_{\alpha}\left(\Omega ;=\lambda_{\alpha} \delta(\Omega)+\rho_{\alpha}(\Omega) .\right.
$$

We assume $\lambda(-\phi)=\lambda(\phi)$, so that for $\alpha=n$, $n$ being an integer,

$$
\lambda_{-n}=\lambda_{n} \text {. }
$$

Let us introduce the following notation:

$$
F(\phi, t)=-2 \pi R\left(1+\frac{\dot{\phi}}{\omega_{0}}\right) \mathscr{E}\left(\omega_{0} t+\phi, t\right) .
$$

This is the energy loss of the particle per turn of revolution due to the beam-induced electric field. This quantity can also be split into two parts corresponding to Eq. (7-23). We call the contribution from $\lambda(\phi)$ to $F$ the incoherent beam loading part; it is

$$
F_{B L}(\phi)=2 \pi I \text { av } \sum_{n=-\infty}^{\infty} \lambda_{n} z_{n}\left(n \omega_{0}\right) e^{i n \phi}
$$

The coherent contribution from $\rho^{(1)}(\phi, t)$ is

$$
F_{s}(\phi, t)=2 \pi I_{a v} \sum_{n=-\infty}^{\infty}\left[d \Omega \rho_{n+\Omega / \omega}(\Omega) z_{n}\left(n \omega{ }_{0}+\Omega\right) e^{i\left(n+\Omega / \omega_{0}\right) \phi-i \Omega t} .\right.
$$

If $\mathscr{F}_{\mathrm{BL}}(\phi)$ is kept only to first order in $\phi$, Eq. (7-28) becomes

$$
\mathscr{F}_{\mathrm{BL}}(\phi)=2 \pi I_{\mathrm{av}}\left(\sum_{n=-\infty}^{\infty} \lambda_{\mathrm{n}} \mathbb{R}_{\mathrm{n}}\left(n \omega_{0}\right)-\phi \sum_{n=-\infty}^{\infty} \pi X_{n}\left(\pi \omega_{0}\right) \lambda_{n}\right),
$$

vihere Eqs. (7-26), (3-18), and (3-19) have been used.

Similarly, we linearly approximate the rf voltage:

$$
\hat{v}_{g} \sin \left(h \phi-\phi_{g}\right)=-\hat{v}_{g} \sin \phi_{g}+h \hat{v}_{g} \phi \cos \phi_{g} .
$$

The equation of motion (7-22) now becomes

$$
\begin{aligned}
\ddot{\phi}= & \frac{e \bar{n}}{2 \pi}\left(\left\{-\hat{v}_{g} \sin \phi_{g}+v_{Y}+v_{E}+2 \pi I_{a v} \sum_{n=-\infty}^{\infty} \lambda_{n} \Re_{n}\left(n \omega_{o}\right)\right\}\right. \\
& \left.+\phi\left\{h \hat{v}_{g} \cos \phi_{g}-2 \pi I_{a v} \sum_{n=-\infty}^{\infty} n z_{n}\left(n \omega_{o}\right) \lambda_{n}+\mathscr{F}_{s}(\phi, t)\right\}\right) .
\end{aligned}
$$

The synchronous phase $\phi_{g}$ of the rf voltage is determined by the following condition: For a synchronous particle $(\phi=0)$, the energy gain of the particle due to acceleration and the energy loss due to synchrotron radiation and beam loading should be exactly balanced by the energy provided by the rf generating voltage. That is, 


$$
\hat{\nabla}_{g} \operatorname{sin\phi _{g}}=\nabla_{Y}+\nabla_{E}+2 \pi I \text { av } \sum_{n=-\infty}^{\infty} \lambda_{n} \Re_{n}\left(n \omega_{0}\right) .
$$

Therefore, the terms within the first $\{\ldots\}$ in Eq. (7-32) cancel. With the definitions

$$
\omega_{\text {so }}^{2}=-\frac{e \bar{n} h}{2 \pi} \hat{v}_{g} \cos \phi_{g},
$$

and

$$
\omega_{s}^{2}=\omega_{s o}^{2}+\bar{n} e I_{a v} \sum_{n=-\infty}^{\infty} n z_{n}\left(n \omega_{o}\right) \lambda_{n},
$$

Eq. (7-32) becomes

$$
\ddot{\phi}+\omega_{s}^{2} \phi=\frac{e \bar{\pi}}{2 \pi} \mathscr{F}_{s}(\phi, t) .
$$

The quantity $\omega_{s o}$ is the synchrotron frequency in the absence of beam loading, and $w_{s}$, which includes the effect of beam loading, is the actual synchrotron frequency of incoherent phase focusing. The righthand side of Eq. (7-36), which is generated by the coherent oscillation of the beam, is in turn the driving force of the coherent motion.

\subsubsection{Hamiltonian formalism}

We now write the equation of motion (7-36) in a Hamiltonian form. We choose $\phi$ and $W=\left(E-E_{0}\right) / E_{0}$ as the canonical coordinate and momentum, respectively; $(\phi, W)$ emerges naturally as a canonical pair in the standard Hamiltonian treatment ${ }^{8}$ of Lorentz force. (See Appendix B.)

Equation (7-36), can be derived from the following Hamiltonian:

with

$$
\mathrm{H}=\mathrm{H}_{\mathrm{O}}+\mathrm{U}^{s}(\phi, t) \text {, }
$$

$$
H_{0}=-\frac{1}{2}\left(\bar{\pi} \omega^{2}+\omega_{s}^{2} \phi^{2} / \bar{\eta}\right) \text {, }
$$

and

$$
\frac{\partial U^{S}(\phi, t)}{\partial \phi}=\frac{e}{2 \pi} \mathscr{F}_{s}(\phi, t) \text {. }
$$

It is convenient to use the action-angle variables $(J, \psi)$ asso-

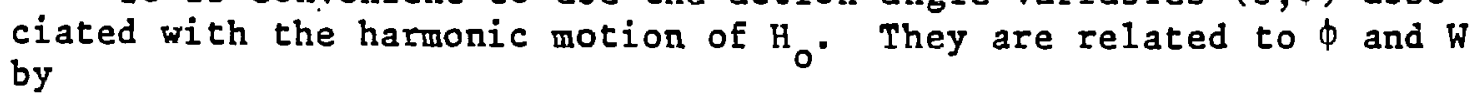

$$
\phi=\bar{\phi} \cos \psi, \quad w=-\omega_{s} \bar{\phi} \sin \psi / \bar{\pi},
$$


where the synchrotron amplitude is

$$
\bar{\phi}=\sqrt{\bar{\eta} \frac{2 J}{\omega_{s}}}
$$

In terms of the action-angle variables,

$$
H_{0}=-\omega s
$$

and

$$
H=-\omega_{s} J+U^{s} \text {. }
$$

\subsection{Vlasov Equation}

The Liouville theorem for a canonical system is

$$
\frac{\partial \Psi}{\partial t}+(\Psi, H)=0
$$

where the Poisson bracket $($,$) is defined by$

$$
(A, B)=\frac{\partial A}{\partial \psi} \frac{\partial B}{\partial J}-\frac{\partial A}{\partial J} \frac{\partial B}{\partial \psi}
$$

and $\Psi$ is the phase space density function.

We refer to $(7-43)$ as the Vlasov equation.

From Eq. (7-42), Eq. (7-43) becomes

$$
\frac{\partial \Psi}{\partial t}-w_{s} \frac{\partial \Psi}{\partial \Psi}+\left(\Psi, \sigma^{s}\right)=0
$$

The following decomposition is useful:

$$
\Psi(J, \Psi, t)=\Psi_{0}(J)+\Psi_{1}(J, \psi, t),
$$

where $\Psi_{0}$ is the equilibrium bunch distribution, and $\Psi_{1}$ is the modification due to the coherent oscillation. These $\Psi$ 's are related to the line densities in Eq. (7-23) by

$$
\begin{aligned}
\rho(\phi, t) & =\int d w^{\Psi}(J, \psi, t), \\
\lambda(\phi) & =\int d w^{\Psi}{ }_{0}(J), \\
\rho{ }^{(1)}(\phi, t) & =\int d w^{\Psi}{ }_{1}(J, \psi, t) .
\end{aligned}
$$

The Vlasov equation now becomes

$$
\frac{\partial \Psi_{1}}{\partial t}-w_{s} \frac{\partial \Psi_{1}}{\partial \Psi}+\left(\Psi_{0}, U^{s}\right)+\left(\Psi_{1}, U^{s}\right)=0 \text {. }
$$


Note that $U^{S}$ is first order in $\Psi_{1}$; therefore, the last term in Eq. $(7-47)$ is second order. Ignoring the last term, we obtain the

linearized $\nabla$ lasov equation

$$
\frac{\partial \Psi_{1}}{\partial t}-\omega_{s} \frac{\partial \Psi}{\partial \Psi}+\left(\Psi_{0}, U^{s}\right)=0 \text {. }
$$

The driving force term of Eq. $(7-48)$ is

$$
\begin{aligned}
\left(\Psi_{0}(J), U^{s}(\phi, t)\right) & =-\Psi_{0}^{\prime}(J) \frac{\partial U^{s}}{\partial \phi} \frac{\partial \phi}{\partial \psi} \\
& =\Phi \sin \psi \Psi_{0}^{\prime}(J) \frac{e}{2 \pi} \pi_{s}(\phi, t),
\end{aligned}
$$

where we have defined

$$
\Psi_{0}^{\prime}(J)=\frac{d}{d J} \Psi_{0}(J)
$$

So the linearized vlasov equation is

$$
\frac{\partial \Psi_{1}(J, \Psi, t)}{\partial t}-\omega_{s} \frac{\partial \Psi}{\partial \Psi}=-\frac{e}{2 \pi} \sin \psi \bar{\phi} \Psi_{0}^{\prime}(J) \widetilde{F}_{s}(\phi, t) \text {. }
$$

Introducing the Fourier component of $\Psi_{1}(J, \psi, t)$ by

$$
\Psi_{1}(J, \psi, t)=\int_{-\infty}^{\infty} d \Omega^{\Psi}(J, \psi, \Omega) e^{-i \Omega, t},
$$

and using Eq. (7-29), we change Eq. (7-50) to

$$
\begin{aligned}
-i \Omega \Psi_{1}(J, \Psi, \Omega)-\omega_{s} & \frac{\partial \Psi_{1}(J, \Psi, \Omega)}{\partial \psi} \\
& =-e I_{a v} \sin \psi \bar{\phi} \Psi_{0}^{\prime}(J) \sum_{n=-\infty}^{\infty} \rho_{\bar{n}} Z_{n} e^{i \bar{n} \Phi \cos \psi,}
\end{aligned}
$$

where we use the short-hand notation,

and

$$
\left.z_{n}=z_{n}(n \omega)+R\right)
$$

$$
\overline{\mathbf{n}}=\mathbf{n}+\Omega / \omega_{0} .
$$

The right-hand side of Eq. $(7-52)$ is periodic in $\psi$. Hence, the equation is equivalent (see $(A-1)$ in Appendix $A$ ) to 


$$
\begin{aligned}
\Psi_{1}(J, \psi, \Omega)= & -\frac{1}{\omega_{s}} \text { eI }{ }_{a v}^{\bar{\phi} \Psi_{0}^{\prime}} \frac{1}{1-e^{i 2 \pi Q}} \sum_{n} \rho_{\bar{n}} Z_{n} \int_{0}^{2 \pi} d \psi^{\prime} e^{i Q \psi^{\prime}} \sin \left(\psi+\psi^{\prime}\right) \\
& \times e^{i \bar{n} \bar{\phi} \cos \left(\psi+\psi^{\prime}\right)}
\end{aligned}
$$

where

$$
Q=\Omega / \omega_{s}
$$

Exercise: Prove by direct substitution that Eq. $(7-55)$ is equivalent to Eq. $(7-52)$.

From Eqs. $(7-51),(7-46 c)$, and $(7-24 b)$,

$$
\begin{aligned}
\rho_{\bar{n}} & =\frac{1}{2 \pi} \int_{0}^{2 \pi} d \phi \int d W \Psi_{1}(J, \psi, \Omega) e^{-i \bar{n} \phi} \\
& =\frac{1}{2 \pi} \int_{0}^{\infty} d J \int_{0}^{2 \pi} d \psi \Psi_{1}(J, \psi, \Omega) e^{-i \bar{n} \phi \cos \psi,} .
\end{aligned}
$$

where we use $d \phi d W=d J d \psi$, which follows from the canonical invariance of the phase space volume. Using Eqs. $(7-57)$ and (7-55), we obtain the secular equation

$$
\rho_{\overline{\mathrm{m}}}(\Omega)=\sum_{\mathrm{n}=-\infty}^{\infty} \mathrm{T}_{\mathrm{mn}}(\Omega) \rho_{\overline{\mathrm{n}}}(\Omega),
$$

where

$$
\begin{aligned}
T_{m n}= & \frac{e I_{a v}}{2 \pi \omega} \frac{z_{n}}{e^{i 2 \pi Q}-1} \int_{0}^{\infty} d J \bar{\phi} \Psi_{0}^{\prime}(J) \int_{0}^{2 \pi} d \psi \int_{0}^{2 \pi} d \psi^{\prime} e^{i Q \psi^{\prime}} \sin \left(\psi+\psi^{\prime}\right) \\
& \times e^{i \bar{n} \bar{\phi} \cos \left(\psi+\psi^{\prime}\right)-i \bar{m} \bar{\phi} \cos \psi}
\end{aligned}
$$

Let us analytically continue (7-58) into the complex $\Omega$-plane. The value of $\Omega$ which satisfies this equation is the coherent frequency of a coherent oscillation, and the corresponding eigenvector $\rho_{\overline{\mathbf{n}}}$ gives the perturbed line density. 


\section{Exercise: Define}

$$
\rho^{(1)}(\phi, \Omega)=\frac{1}{2 \pi} \int_{-\infty}^{\infty} \mathrm{d} t \rho(1)(\phi, t) \mathrm{e}^{i \Omega t}
$$

and prove that, for $0<\phi<2 \pi$,

$$
\rho^{(1)}(\phi, \Omega)=\sum_{n=-\infty}^{\infty} \rho_{n+\Omega / \omega_{0}} e^{i\left(n+\Omega / \omega_{0}\right) \phi}
$$

The matrix element can be written (for a useful table of integrals see Appendix A) as

$$
\begin{aligned}
T_{m n}= & -i \frac{e I_{a v}}{\omega_{s}} \frac{\bar{m} z_{n}}{e^{i 2 \pi Q}-1} \int_{0}^{\infty} d J \bar{\phi} \psi_{0}^{\prime}(J) \int_{0}^{2 \pi} d \psi^{\prime} e^{i Q \psi^{\prime}} \\
& \times \frac{\sin \psi^{\prime}}{\sqrt{\bar{\pi}^{2}+\bar{m}^{2}-2 \bar{\pi} \cos \psi^{\prime}}} J_{1}\left(\sqrt{\bar{\pi}^{2}+\overline{\mathbf{m}}^{2}-2 \pi \bar{m} \cos \psi^{\prime}} \bar{\phi}\right) .
\end{aligned}
$$

The rest of Part I will be based on the assumption that the equilibrium distribution has a Gaussian form,

$\Psi_{0}(J)=\frac{1}{2 \pi} \frac{\bar{\eta}}{\omega_{S} \sigma_{\phi}^{2}} e^{-\bar{\phi}^{2} / 2 \sigma_{\phi}^{2}}=\left(\frac{1}{\sqrt{2 \pi} \sigma_{\phi}} e^{-\phi^{2} / 2 \sigma_{\phi}^{2}}\right)\left(\frac{1}{\sqrt{2 \pi} \sigma_{W}} e^{-W^{2} / 2 \sigma_{W}{ }^{2}}\right)$,

where $\sigma_{W}=\omega_{s} \sigma_{\phi} / \bar{\eta}$. The coefficient above is chosen so that

$$
\int \mathrm{d} J d \psi \dddot{\varpi}_{0}(\mathrm{~J})=1 \text {. }
$$

Upon substitution of Eq. (7-6i) into (7-60); the integral in $\mathrm{J}$ in the resulting equation can be performed, and the matrix element becomes

$$
\begin{aligned}
T_{\operatorname{man}}= & -i \frac{\bar{n}}{2 \pi \omega_{s}^{2}} \text { eI } a v \frac{\overline{\mathrm{m} Z} \mathrm{n}}{1-e^{i 2 \pi Q}} e^{-\left(\bar{n}^{2}+\bar{\pi}^{2}\right) \sigma_{\phi}^{2} / 2} \\
& \times \int_{0}^{2 \pi} d \psi e^{i Q \psi} \sin \psi e^{\overline{\bar{m}} \cos \psi \sigma_{\phi}^{2}} .
\end{aligned}
$$

This expression can be transformed into a modified Bessel series representation. From Eq. (A-10), 
$I_{m n}=i \frac{\bar{n}}{\pi \omega_{s}^{2} \sigma_{\phi}^{2}}$ eI $\frac{z_{n}}{\bar{n}} \sum_{\mu=1}^{\infty} \frac{\mu^{2}}{Q^{2}-\mu^{2}} I_{\mu}\left(\bar{m} \bar{n} \sigma_{\phi}^{2}\right) e^{-\left(\bar{n}^{2}+\bar{m}^{2}\right) \sigma_{\phi}^{2} / 2}$

To summarize Section 7: The collective motion of the beam is governed by the vlasov equation (7-43). For a Gaussian bunch, the linearized vlasov equation is equivalent to the secular equation (7-58) with the matrix element given by (7-62) or (7-64). A solution $\Omega=Q \omega_{s}$ of Eq. (7-58) gives the coherent frequency of a collective mode, and the eigenvector describes the corresponding perturbation of the line density.

\section{SYNCHROTRON MODES}

We discussed in Section 6 the Robinson instability of a rigid point-like bunch oscillating around the synchronous point. We shall generalize this discussion to include the effects of possible bunch shape distortion. Landau damping will be ignored. 34

Our starting point is Eqs. (7-58) and (7-64).

The condition for a coherent mode is that the matrix $\left(T_{\mathrm{mn}}\right)$ has 1 as one of its eigenvalues. This condition cannot be satisfied if $\left|\mathrm{T}_{\mathrm{mn}}\right| \ll 1$ for all $\mathrm{m}$ and $\mathrm{n}$.

We consider here a situation in which the beam current and/or the impedance is so small that

$$
x_{n}=\frac{\bar{n}}{\pi \omega_{s}^{2} \sigma_{\phi}^{2}} \text { eI } \operatorname{av}_{n} / \bar{n} \ll 1
$$

for all n. Then, from Eq. (7-64) and the above reasoning, there cannot be coherent instability unless $Q=\mu$ for some $\mu$, or $\Omega \simeq \mu \omega_{s}$. The coherent mode satisfying this condition is called the $\mu-t h$ synchrotron mode. $\mu=1,2,3, \ldots$ modes are, respectively, called dipole, quadrupole, sextupole,... modes. It will be seen below that the Robinson instability corresponds to the dipole synchrotron mode. mode:

We adopt the following approximation of $\mathrm{Eq} .(7-64)$ for the $\mu-t h$

$$
T_{m n}=i \frac{\bar{\eta}}{\pi \omega_{s}^{2} \sigma_{\phi}^{2}} e I_{a v}\left(z_{n} / \bar{n}\right) \frac{\mu^{2}}{Q^{2}-\mu^{2}} I_{\mu}\left(m \bar{n} \sigma_{\phi}^{2}\right) e^{-\left(n^{2}+m^{2}\right) \sigma_{\phi}^{2} / 2} .
$$

Let us consider the case in which the bunch length is shorter than the wavelengths of the perturbing EM fields, Then the argument of the modified Bessel function is a small quantity. Using

$$
I_{\mu}\left(\overline{\mathrm{m}} \overline{\mathrm{n}} \sigma_{\phi}^{2}\right) \simeq \frac{1}{\mu ! 2^{\mu}}\left(\overline{\mathrm{m}} \overline{\mathrm{n}} \sigma_{\phi}^{2}\right)^{\mu},
$$

we can approximate $\mathrm{Eq} \cdot(8-2)$ as 


$$
T_{\mathrm{mn}}=i \frac{\bar{\eta}}{\pi \omega_{s}^{2} \sigma_{\phi}^{2}} \text { eI } \frac{\sigma_{\phi}^{2 \mu}}{\mu ! 2^{\mu}} \frac{\mu^{2}}{Q^{2}-\mu^{2}} \frac{z_{n}}{\bar{n}}(\bar{m} \bar{n})^{\mu} e^{-\left(\bar{n}^{2}+\bar{m}^{2}\right) \sigma_{\phi}^{2} / 2} .
$$

The matrix represented by Eq. (8-3) is of rank 1 (factorized in m and $n$ ); hence Eq. (7-58) can easily be diagonalized. The result is

with

$$
\Omega^{2}=\mu^{2} \omega_{s}^{2}+i \frac{\bar{\pi}}{\pi} \text { eI } \frac{\mu}{(\mu-1) ! 2^{\mu}} \sigma_{\phi}^{2 \mu-2} z_{\text {eff }}^{(\mu)},
$$

$$
z_{\text {eff }}^{(\mu)}=\sum_{n=-\infty}^{\infty}\left(n+\frac{\Omega}{\omega_{0}}\right)^{2 \mu-1} z_{n}\left(n \omega_{0}+\Omega\right) e^{-\pi^{-2} \sigma_{\phi}^{2}},
$$

where Eq. (7-54) has been used.

Note that Eqs. $(8-4)$ and $(8-5)$ reduce to Eq. $(6-15)$ of Robinson instability if $\mu=1$ and $\sigma_{\phi}=0$.

We observe that if $\Omega$ is a solution of Eq. (8-4), then so is $-\Omega^{*}$. This follows from the symmetry property $(3-17)$ of the impedance. The two solutions have equal imaginary parts, and their real parts are equal in magnitude but opposite in sign. Therefore, we lose no generality in assuming $\Omega$ to have a non-negative real part.

Let us approximate $\mathrm{E}_{4} .(8-4)$ by

$\Omega^{2}=\mu^{2} \omega_{s}^{2}+i \frac{\bar{n}}{\pi}$ eI $\frac{\mu}{(\mu-1) ! 2^{\mu}} \sigma_{\phi}^{2 \mu-2} \sum_{n=-\infty}^{\infty} n^{2 \mu-1} z_{n}\left(n \omega_{0}+\mu \omega_{s}\right) e^{-n^{2} \sigma_{\phi}^{2}}$ (8-6)

Then, the stability condition is

$$
\begin{aligned}
n \sum_{n=-\infty}^{\infty} n^{2 \mu-1} \mathscr{R}_{n}\left(n \omega_{0}+\mu \omega_{s}\right) e^{-n^{2} \sigma_{\phi}^{2} / 2} \\
\quad=\pi \sum_{n=1}^{\infty} n^{2 \mu-1}\left(\mathscr{R}_{n}\left(n \omega_{0}+\mu \omega_{s}\right)-\mathscr{R}_{n}\left(n \omega_{0}-\mu \omega_{s}\right)\right)<0 .
\end{aligned}
$$

The conventional way of ensuring stability against the single bunch synchrotron modes is by detuning the rf cavity. As can be seen from Eq. (8-7), the contribution to $\operatorname{Im}(\Omega)$ comes predominantly from the part of the impedance that varies appreciably in a frequency range of the order of synchrotron frequency. The rf system is generally the most important source of such an impedance.

Let us consider the case $n>0$ (above transition). The resistive part $\mathscr{R}(\omega)$ of the impedance peaks at the rf frequency $\omega_{\tau f} / 2 \pi$. Therefore, if we tune the rf cavity so that $\omega_{r f}<h \omega_{0}$, then, from Eq. (8-7), the impedance from the rf-cavity fundamental contributes a damping term to the coherent frequency. Such a procedure is called Robinson damping.

Below transition, the rf-cavity should be detuned in the opposite way, $\omega_{\text {If }}>h \omega_{0}$. 
If there are many identical bunches symmetrically arranged around the ring, then, as will be seen in Section 11, there will be coherent modes to which the rf fundamental impedance does not contribute. Hence, Robinson detuning is not effective against these modes.

\section{LONGITUDINAL STRONG COUPLING - SHORT BUNCH CASE}

We saw in Section 8 that under the condition $X_{n} \ll 1$ for all $n$ (see $(8-1)$ ), the coherent modes can be classified according to the harmonic number $\mu$ of the synchrotron frequency. The coherent freqency $\Omega$ corresponding to the synchrotron mode $\mu$ satisfies $\Omega \bumpeq \mu \omega_{s}$. This is no longer true if $X_{n} \geqslant 1$ for some $n$ 's. In such a case, the matrix $\left(T_{m n}\right)$ may have 1 as one of its eigenvalues without $Q$ being close to an integer; thus many terms in the summation of Eq. (7-64) may contribute with comparable strength to a coherent mode. When this occurs, $\mu$ ceases to be the mode number for an eigenmode.

We also saw that, when the bunch length is small compared with the perturbing EM wavelength, we can diagonalize the matrix (8-2) for the $\mu-t h$ synchrotron mode by approximating it with a matrix of rank 1 . Here we generalize this method to the $X_{n} \geqslant 1$ case when the bunch is short. Our method consists of expanding Eq. (7-64) in an asymptotic series of small parameters $\mathrm{m}_{\phi}$ and $\mathrm{n} \sigma_{\phi}$, and thereby approximating the -dimensional matrix $\left(T_{m n}\right)$ by a matrix of finite rank. A matrix of finite rank can be diagonalized with an elementary algebraic procedure. The long bunch case will be treated in the next section. equation

We recall that the coherent modes are determined by the secular

$$
\rho_{\mathrm{m}}=\sum_{\mathrm{n}=-\infty}^{\infty} T_{\mathrm{mn}}(\Omega) \rho_{\mathrm{n}}
$$

with

$$
\begin{aligned}
T_{\operatorname{mn}} & =i \chi_{n} \sum_{\mu=1}^{\infty} \frac{\mu^{2}}{Q^{2}-\mu^{2}} I_{\mu}\left(\overline{\bar{m}} \bar{n} \sigma_{\phi}^{2}\right) e^{-\left(\overline{\mathrm{m}}^{2}+n^{2}\right) \sigma_{\phi}^{2} / 2}, \\
\overline{\mathrm{n}} & =\mathrm{n}+\Omega / \omega_{0}, \quad Q=\Omega / \omega_{s}, \\
\chi_{\mathrm{n}} & =\frac{1}{m_{\mathrm{s}}^{2} \sigma_{\phi}^{2}} \bar{n}_{\mathrm{eI}} \mathrm{av}_{\mathrm{n}} / \overline{\mathrm{n}} .
\end{aligned}
$$

Let us expand the modified Bessel function in $24 .(9-2)$ in Taylor series. Then, after recombining the terms, the matrix element becomes

$$
\mathrm{T}_{\mathrm{mn}}=i \chi_{\mathrm{n}} \mathrm{e}^{-\left(\overline{\mathrm{n}}^{2}+\overline{\mathrm{m}}^{2}\right) \sigma_{\phi}^{2} / 2} \sum_{\ell=1}^{\infty} a_{\ell}\left(\overline{\mathrm{m}} \pi \sigma_{\phi}^{2}\right)^{\ell}
$$

where 


$$
\begin{aligned}
& a_{1}=\frac{1}{2} \frac{1}{Q^{2}-1}, \quad a_{2}=\frac{1}{2} \frac{1}{Q^{2}-4}, \quad a_{3}=\frac{1}{16}\left(\frac{1}{Q^{2}-1}+\frac{3}{Q^{2}-9}\right), \\
& a_{4}=\frac{1}{24}\left(\frac{1}{Q^{2}-4}+\frac{1}{Q^{2}-16}\right), \text { etc. }
\end{aligned}
$$

Each term in (9-5) is factored into the product of a function of $m$ and a function of $\mathrm{n}$. Let us perform the following change of base:

$$
\bar{\rho}_{\ell}=i \sum_{n=-\infty}^{\infty} x_{n} e^{-\bar{n}^{2} \sigma_{\phi}^{2} / 2}\left(\bar{n} \sigma_{\phi}\right)^{l} \rho_{n} .
$$

Then $(9-1)$ becomes

$$
\bar{\rho}_{\ell}=\sum_{\ell^{\prime}=1}^{\infty} \overline{\mathrm{T}}_{\ell \ell}, \bar{\rho}_{\ell},
$$

with

$$
\begin{aligned}
\overline{\mathrm{T}}_{\ell \ell^{\prime}} & =a_{\ell^{\prime}} \mathscr{F}_{\ell+\ell^{\prime}}, \\
\mathscr{F}_{\ell} & =i \sum_{n=-\infty}^{\infty} \chi_{n} \mathrm{e}^{-\mathrm{n}^{-2} \sigma_{\phi\left(\bar{n} \sigma_{\phi}\right)^{\ell}} .}
\end{aligned}
$$

We observe that Eq. $(9-10)$ is, up to a constant, the same as the last term of Eq. $(8-4)$.

Equation (9-8) provides a convenient starting point for treating the coherent motion of a small bunch. We assume that there, exists a number $n_{\max }, n_{\max } \sigma_{\phi}<1$, such that $\chi_{\mathbf{n}}$ is negligible if $|n|>n_{\max }$. Then $\bar{T}_{\ell \ell}$ ' decreases with increasing $\ell$ and $\ell^{\prime}$, and hence Eq. $(9-8)^{\text {max }}$ can be truncated at $l, \ell^{\prime}=l_{\max }$, where $\ell_{\max }$ is determined by $n_{\max } \sigma_{\phi}$. Now Eq. $(9-8)$ becomes

$$
\bar{\rho}_{\ell}=\sum_{\ell=1}^{\ell} \overline{\mathrm{T}}_{\ell \ell}, \bar{\rho}_{\ell} .
$$

This is a secular equation in a finite-dimensional vector space, and the coherent frequency $\Omega$ can now be determined algebraically,

$$
\operatorname{det}\left(\bar{T}_{\ell \ell^{\prime}}-\delta_{\ell_{\ell}{ }^{\prime}}\right)=0 \text {. }
$$

We illustrate the above method by the case where $l_{\max }=2$. Equation $(9-12)$ becomes

$$
4 Q^{2}=10+\mathscr{F}_{2}+\mathscr{F}_{4} \pm \sqrt{\text { Discr }}
$$

where the discriminant is 


$$
\text { Discr }=36-12 \mathscr{F}_{2}+12 \mathscr{F}_{4}+\left(\mathscr{F}_{2}-\mathscr{F}_{4}\right)^{2}+4 \mathscr{F}_{3}^{2} \cdot(9-14)
$$

First, let us discuss the limit of Eq: $(9-13)$ when $X_{n} \ll 1$. Then, to the first order in $F$, the equation becomes

$$
Q^{2}=1+\frac{1}{2} \mathscr{F}_{2} \quad \text { or } \quad 4+\frac{1}{2} \mathscr{F}_{4} \text {. }
$$

This is the synchrotron mode result $(8-4)$ with $\mu=1$ or 2 . As we recall, these instabilities can be Robinson damped.

When $X ' s$ are not smal $\perp$, computer studies indicate that unstable solutions may emerge from Eq. (9-13) even if the individual $\mathscr{F}$ 's are Robinson damped.

\section{LONGITUDINAL STRONG COUPLING - LONG BUNCH CASE}

We have analyzed the coupling of the synchrotron modes for the case in which the wavelengths of the perturbing EM fields are longer than the bunch length. Here we study the opposite, short EM wavelength, case. In particular, it will be demonstrated that, in the limit where the growth rate of the instability is much greater than the synchrotron frequency, the bunched beam instability is very much like that of a coasting beam. In other words, the equations that govern the coherent behavior will reduce to those discussed in Section 5 .

From the point of view of the synchrotron modes, what distinguishes the long bunch (or short perturbing EM wavelengths) from the short bunch is that, when $X_{\mathrm{n}} \geqslant 1$, many more synchrotron modes contribute to an eigenmode for the long bunch case. Let $a_{\max }$ be the revolution mode number beyond which $z_{n} / n$ is negligible. We can readily see from Eq. (7-64) that, if $\chi_{n} \leqslant 1$, the number of synchrotron modes that couple to form a coherent state is $\Omega_{\max }{ }^{2} \sigma_{\phi}^{2}$, which is large for a long bunch. A coherent state is a fast blowup state if its coherent frequency $\Omega$ satisfies

$$
\operatorname{Im}(Q)=\operatorname{Im}(\Omega) / \omega_{S} \gg 1 .
$$

We shall find the condition under which Eq. (10-1) is satisfied.

Our starting point is the secular equations (7-58) and (7-63). We shall ignore the distinction between $\bar{n}=n+\Omega / \omega_{0}$ and $n$.

$$
\begin{gathered}
\rho_{m}=\sum_{n=-\infty}^{\infty} T_{m n} \rho_{n}, \\
T_{m n}=-i \frac{\bar{n}}{2 \pi \omega_{s}^{2}} e I_{a v} \frac{m z_{n}}{1-e^{i 2 \pi Q}} \int_{0}^{2 \pi} d \psi e^{i Q \psi} s i n \psi \\
\times e^{-\left(n^{2}+m^{2}-2 n m \cos \psi\right) \sigma_{\phi}^{2} / 2} .
\end{gathered}
$$


Let us take the asymptotic limit (10-1) of the matrix element $(10-3)$. We first note that

$$
\frac{1}{1-e^{i 2 \pi Q}}+1 \text {. }
$$

Recall that this factor was the origin of the synchrotron poles at $Q=\mu, \mu= \pm 1, \pm 2, \ldots$, in Eq. $(7-64)$. Thus the synchrotron modes lose their significance completely in the fast blowup limit (10-1). Next we investigate the long bunch limit (high frequency limit),

$$
\ln \left|\sigma_{\phi}, \quad\right| \mathrm{m} \mid \sigma_{\phi} \gg 1
$$

together with the limit (10-1) of the integral in Eq. (10-3). Under (10-1), the integral is dominated by the contribution from the integration region in the neighborhood of $\psi=0$. Therefore

$$
\begin{aligned}
\int_{0}^{2 \pi} d \psi e^{i Q \psi} & \sin \psi e^{-\left(n^{2}+m^{2}-2 n m \cos \psi\right) \sigma_{\phi}^{2} / 2} \\
& \approx e^{-(n-m)^{2} \sigma_{\phi}^{2} / 2} \int_{0}^{2 \pi} d \psi e^{i Q \psi} e^{-n m \sigma} \phi^{2} \psi^{2} / 2 .
\end{aligned}
$$

If $n$ and $m$ are of opposite signs, the exponential factor $\exp \left(-(n-m)^{2} \sigma_{\phi}^{2} / 2\right)$ becomes vanishingly small because of $(10-5)$. Thus, revolution modes with positive and negative $n$ decouple in the high frequency fast blowup limit. Let us consider the positive case where both $n$ and $m$ are positive; the "negative case" is trivially related to the "positive case."

Because of $(10-5)$, the upper limit of integration of Eq. (10-6) can now be replaced by $\infty$. We obtain

$$
\int_{0}^{2 \pi} d \psi e^{i Q \psi} \psi e^{-n m \psi^{2} \sigma_{\phi}^{2} / 2} \simeq \frac{1}{n m \sigma_{\phi}^{2}} h_{L}\left(\frac{1}{\sqrt{n m} \sigma_{\phi}}\right),
$$

with

$$
h_{L}(x)=\int_{0}^{\infty} d \tau e^{i x \tau} e^{-\tau^{2} / 2}
$$

We recall that this function has already beer discussed in connection with the coasting beam instability in section 4.

Combining Eqs. $(10-3)$ to $(10-7)$, we obtain

$T_{m n}=\frac{\bar{n}}{2 \pi \omega_{s}^{2} \sigma_{\phi}^{2}}$ eI $\frac{z_{n}}{n} e^{-(n-m)^{2} \sigma_{\phi}^{2} / 2} h_{L}\left(\frac{\Omega}{\sqrt{n m} \omega_{s} \sigma_{\phi}}\right)$.

Recalling 


$$
\bar{n}=n \frac{\omega_{o}^{2}}{\beta_{0}^{2} E_{0}},
$$

and

$$
\omega_{s} \sigma_{\phi}=\sigma_{\dot{\phi}}=\ln \mid \omega_{\circ} \sigma_{\delta}
$$

Eq. (10-9) can also be written as

$$
T_{\operatorname{mn}}=-i \frac{1}{2 \pi \beta_{0}^{2} E_{0} \sigma_{\delta}^{2}} \notin I_{a v} \frac{z_{n}}{n} e^{-(n-m)^{2} \sigma_{\phi}^{2} / 2} h_{L}\left(\frac{\Omega}{\sqrt{n m}|n| \omega_{0} \sigma_{\delta}}\right) \text {. }
$$

It may be worthwhile to remind ourselves that, for the Gaussian bunch (7-61) under consideration, the line density is

$$
\lambda(\phi)=\frac{1}{\sqrt{2 \pi \sigma_{\phi}}} e^{-\phi^{2} / 2 \sigma_{\phi}^{2}},
$$

and its Fourier component is

$$
\lambda_{n}=\frac{1}{2 \pi} e^{-n^{2} \sigma_{\phi}^{2} / 2}
$$

Since the fractional momentum deviation $\delta$ is proportional to $W$, and (7-61) implies a Gaussian distribution in $W$, the distribution function in $\delta$ should also be Gausian,

$$
g(\delta)=\frac{1}{\sqrt{2 \pi \sigma} \delta} e^{-\delta^{2} / 2 \sigma_{\delta}^{2}}
$$

We now show that the above matrix (10-12) is the same matrix that appeared in the secular equation (5-5).

From Eq. $(10-14)$ and the identity

$$
\int_{-\infty}^{\infty} \mathrm{d} \delta \frac{g^{\prime}(\delta)}{\Omega+\sqrt{\mathrm{mn}} \omega \omega_{0} \delta^{\prime}}=-\frac{1}{\sqrt{\mathrm{mn} \omega} \omega_{0}^{n \sigma_{\delta}^{2}}} h_{L}\left(\frac{\Omega}{\sqrt{\mathrm{mn}}|\eta| \omega_{0} \eta_{\delta}}\right)
$$

which was proven in Section 4 (see (4-22)), Eq. (10-12) becomes

$$
T_{m n}=i \frac{\omega_{0}}{\beta_{0}^{2} E_{0}} \text { eI av } \sqrt{\frac{m}{n}} z_{n} \lambda_{m-n} \int_{-\infty}^{\infty} d \delta \frac{g^{\prime}(\delta)}{\Omega+\sqrt{m n} \omega_{0} n \delta} .
$$

Note that because of the bunch factor $\lambda_{m-n}, T_{m n}$ is vanishingly small unless $|n-m| \sigma_{\phi}$ is of the order of 1 or smaller. Thus, 


$$
\sqrt{\frac{m}{n}}=\frac{m}{n}\left[1+0\left(\frac{1}{n \sigma_{\phi}}\right)\right] \text { and } \operatorname{mn}=n\left[1+0\left(\frac{1}{n \sigma_{\phi}}\right)\right] \text {. }
$$

Therefore the seculä equation here is equivalent to that of Section 5 in the high frequency limit under consideration.

Let us conclude this section by comparing the treatments here and in Section 5. In Section 5, we demonstrated the Boussard conjecture on microwave instability by establishing the dispersion relation (5-5) for general line density $\lambda(\phi)$ and momentum distribution $g(\delta)$ under the assumption of a vanishing synchrotron frequency. Here we proved the same conjecture keeping $\omega_{s}$ finite; however, the proof applies only to the case of Guassian distribution (7-61). Also, recall that the treatment here is based on the assumption of a harmonic rf potential (7-38). For a more general rf-potential, the proof of this section can be carried over ${ }^{19}$ to the case of the corresponding Maxwel1Baltzmann distribution.

\section{LONGITUDINAL SYMMETRIC COUPLED BUNCA MODES}

We treated the single bunch synchrotron mode in Section 8 . Here we consider how the presence of many bunches in the ring affects that treatment. We assume $h$ identical bunches symmetrically distributed around the ring. The conclusion will be that corresponding to, each synchrotron mode number $\mu$, there are $h$ independent coherent modes, each characterized by the way the coherent phases of various bunches are related.

We shall rely heavily on the discussion of Sections 7 and 8 , which need only minor modification for adaptation to the present multibunch case. We sketch the needed modification below.

Denote by $\phi_{j}$ the location of the center of the $j$-th bunch,

$$
\phi_{j}=\frac{2 \pi}{h} \mathrm{j}, \quad \mathrm{j}=0,1, \ldots, \quad \mathrm{h}-1 .
$$

If $\Psi(j)$ is the distribution function of the $j-t h$ bunch, the total distribution function is

$$
\Psi(\phi, W, t)=\sum_{j=0}^{h-1} \Psi^{(j)}\left(\phi-\phi_{j}, W, t\right)
$$

Since different bunches do not overlap in the phase space, the vlasov equation can be written as

$$
\frac{\partial \Psi^{(j)}}{\partial t}+\left(\Psi^{(j)}, H^{(j)}\right)=0, \quad j=0, \ldots, \quad h-1
$$

where

$$
H^{(j)}=-\frac{1}{2}\left(\bar{\eta} \omega^{2}+\omega_{s}^{2}\left(\phi-\phi_{j}\right)^{2} / \bar{\eta}\right)+U^{s}(\phi, t)
$$


with

$$
\begin{gathered}
\frac{\partial v^{(s)}}{\partial \phi}=\frac{e}{2 \pi} \mathscr{F}_{s}(\phi, t), \\
\mathscr{F}_{s}(\phi, t)=-2 \pi R\left(1+\frac{\phi}{\omega_{0}}\right) \mathscr{E}\left(\omega_{0} t+\phi, t\right) .
\end{gathered}
$$

The right-hand side of Eq. $(11-6)$ is averaged in the sense of section 7.1 .1 ; hence it is actually independent of $\phi$. For particles in the bunch $j$,

$$
\phi-\phi_{j}=\bar{\phi} \cos \psi_{s}, \quad W=-\omega_{s} \bar{\phi} \sin \psi / \bar{n}
$$

with

$$
\bar{\phi}=\bar{\phi}(\mathrm{J})=\sqrt{\bar{\eta} \frac{2 J}{\omega_{s}}} .
$$

We adopt the following normalization:

$$
\int d \phi d W \Psi^{(j)}=\int d J d \psi \Psi^{(j)}=1,
$$

and, instead of inventing a new notation, write

$$
\Psi^{(j)}(J, \psi, t)=\psi^{(j)}\left(\phi-\phi_{j}, W^{\prime}, t\right) .
$$

Let us decompose

$$
\Psi^{(j)}=\Psi_{0}(J)+\Psi_{1}^{(j)}(J, \psi) e^{-i \Omega_{1}} .
$$

The independence of $\Psi_{0}$ from $j$ follows from the assumption that the bunches are identical. The perturbed line density of the beam is

$$
\rho_{1}(\phi) e^{-i \Omega t}=\sum_{j} \int \mathrm{d} J d \psi \delta\left(\phi-\phi_{j}-\bar{\phi}_{\cos \psi) \Psi_{1}}{ }^{(j)}(J, \psi) e^{-i \Omega t} .\right.
$$

Introduce the notation

and define

$$
\vec{n}=\mathbf{n}+\Omega / \omega_{0},
$$

$$
\begin{aligned}
\rho_{\bar{n}} & =\frac{1}{2 \pi} \int_{0}^{2 \pi} d \phi \rho_{1}(\phi) e^{-i \bar{n} \phi} \\
& =\sum_{j} \rho \frac{(j)}{n} e^{-i \bar{n} \phi} j
\end{aligned}
$$

with 


$$
\rho_{\bar{a}}^{(j)}=\frac{1}{2 \pi} \int \mathrm{dJ} d \psi \delta\left(\phi-\phi_{j}-\bar{\phi} \cos \psi\right) \Psi_{1}^{(j)}(J, \psi) e^{-i \bar{n}\left(\phi-\phi_{j}\right)} .
$$

The energy loss of the particle per revolution can now be written as

$$
\begin{aligned}
\mathscr{F}_{s}(\phi, t) & =2 \pi I_{B} \sum_{n} \rho_{\bar{n}} Z\left(n \omega_{0}+\Omega\right) e^{i \bar{n} \phi-i \Omega t} \\
& =2 \pi I_{B} \sum_{k=0}^{h-1} \sum_{n} \rho_{n}^{(j)} z\left(n \omega_{0}+\Omega\right) e^{i \bar{n}\left(\phi-\phi_{k}\right)-i \Omega t}
\end{aligned}
$$

where $I_{B}$ is the average current per bunch, $I_{B}=I_{a v} / h$.

Using the technique introduced in Section 7 , we obtain -- from Eqs. $(11-3)$ to $(11-5),(11-10),(11-15)$, and $(11-16)-$

$$
\rho_{\text {in }}^{(j)}=\frac{1}{h} \sum_{k=0}^{h-1} \sum_{n=-\infty}^{\infty} T_{m n} e^{i \bar{n}\left(\phi_{j}-\phi_{k}\right)} \rho_{\pi}^{(k)},
$$

where for Gaussian bunches,

$$
\begin{aligned}
T_{\mathrm{mn}}= & -i \frac{\bar{n}}{2 \pi \omega_{s}^{2}} \text { eI } \frac{\overline{\mathrm{m}} z_{\mathrm{n}}}{1-\mathrm{e}^{i 2 \pi Q}} e^{-\left(\tilde{n}^{2}+\overline{\mathrm{m}}^{2}\right) \sigma_{\phi}^{2} / 2} \\
& \times \int_{0}^{2 \pi} d \psi \mathrm{e}^{i Q \psi_{s i n} \psi \mathrm{e}^{-\bar{n} \cos \psi \sigma_{\phi}^{2}} .}
\end{aligned}
$$

Note that $(11-18)$ is the same as $(7-63)$.

We approximate the phase factor in $(11-17)$ by

$$
e^{i \bar{n}\left(\phi_{j}-\phi_{k}\right)}+e^{i n\left(\phi_{j}-\phi_{k}\right)}
$$

This amounts to approximating the phase shift between two particles in different bunches by the phase shift between the corresponding synchronous particles.

The $h \times h$ matrix with its element given by (11-9) can be diagonalized easily. The result as applied to Eq. (11-17) is

$$
\rho_{\overline{\mathrm{m}}}^{(\mathrm{j})}=\rho_{\overline{\mathrm{m}}}^{(0)} \mathrm{e}^{\mathrm{i} \frac{2 \pi}{\mathrm{h}} \mathrm{sj}}, \quad \mathrm{j}=0,1, \ldots, \quad \mathrm{h}-1,
$$

where the symmetric coupled bunch mode number $s, s=0,1, \ldots, h-1$, characterizes different eigensolutions.

We observe that 


$$
\sum_{k=0}^{h-1} e^{i \frac{2 \pi}{h}(j-k)(n-s)}=h \sum_{\ell=-\infty}^{\infty} \delta_{n-s, l h},
$$

where $\delta_{m, n}$ is Kronecker's delta. From Eqs. (11-20), (11-21), and $(11-19)$. Eq. $(11-17)$ reduces to

$$
\rho_{\overline{\mathrm{mh}}+\mathrm{S}}^{(0)}=\sum_{\mathrm{n}=-\infty}^{\infty} T_{\mathrm{mh}+\mathrm{S}, \mathrm{nh}+\mathrm{S}} \rho_{\overline{\mathrm{n} h}+\mathrm{S}}^{(0)} .
$$

Except for the modification of the subscripts, this secular equation is the same as that of the single bunch case (7-58).

Let us apply Eq. (11-22) to the synchrotron mode; then, for the mode $S$, the coherent frequency $\Omega$ in the small bunch approximation is given by

$$
\begin{aligned}
\Omega^{2}= & \mu w_{s}^{2}+i \frac{1}{\pi} \bar{n}_{e} I_{a v} \frac{\mu}{(\mu-1) ! 2^{\mu}} \sigma_{\phi}^{2 \mu-2} \sum_{n=-\infty}^{\infty}(\bar{n} h+s)^{2 \mu-1} \\
& \times 2\left((n h+s) \omega_{0}+\Omega\right) e^{-(\bar{n} h+S)^{2} \sigma_{\phi}^{2}} .
\end{aligned}
$$

\section{TRANSVERSE INSTABILITIES}

\section{OUTLINE OF PART II}

The discussion of transverse instabilities in Part II will parallel the discussion of the longitudinal version in Part $I$. We assume that the instabilities in the $x$ and $y$ directions are decoupled and discuss only the $y$-instabilities; the discussion of the $x$-instabilities would be totally similar. We use many of the notations defined in Part I; these are listed under "Principal Symbols" at the end of this paper.

In Section 13, we introduce the transverse impedance function $\mathrm{z}^{\mathrm{y}}(\omega)$ which relates the $\mathrm{y}$-component of the Lorentz force field to the dipole density of the beam.

In Section 14, we discuss the transverse coasting beam instability; the approach adopted is that of Courant. ${ }^{35}$ The rest of Part II is devoted to bunched beam instabilities. Single bunch instabilities are treated in Sections 15 to 19 and coupled bunch instabilities ${ }^{4,23}$ in Section 20.

We consider the high frequency bunched beam transverse instability in Chapter 15 under the assumption that the beam is not

longitudinally focused; that is, $\omega_{s}=0$. The results are quite similar to those of the coasting beam case.

The finiteness of the synchrotron frequency of a bunched beam is fully taken into account in the rest of Part II. To this end we introduce in Section 16 the Vlasov equation, which describes the transverse as well as the longitudinal motion of the particles. We also introduce 
a method 17,18 of solving the Vlasov equation within a linear approximation, which is followed in the rest of Part II.

When the coupling between the beam and the EM fields it induces through the impedance is weak, the possible bunched beam transverse coherent instabilities are the head-tail modes. 36,37 For these modes, the coherent frequencies lie very close to the synchrotron sidebands $\mu \omega_{s}, \mu=0, \pm 1, \ldots$. Section 17 is devoted to head-tail modes.

When the beam couples strongly to the EM fields it generates, head-tail modes no longer suffice to classify the transverse bunched beam instabilities. 25 The coherent frequencies in this case may not lie close to a synchrotron sideband, and therefore many head-tail modes couple to form coherent modes. If the bunch length is shorter than the wavelength of the perturbing EM fields, the number of headtail modes which may couple is small. We introduce in section 18 a method 26,18 of treating such short bunch instabilities. In Section 19, we treat the strong coupling instabilities in the long bunch case.17,18 We find that in the further limit of growth rate $>$ synchrotron frequency, the results recuce to those of Section 15.

We provide a mathenatical table: a Appendix A. In Appendix B, we demonstrate how the Hamiltonian used in Section 16 can be obtained from a series of canonical transformations of the fundamental Hamiltonian of the Lorentz force. The discussion is restricted for simplicity to a weak focussing storage ring.

The transverse coherent instability derives the energy it needs from the longitudinal orbit motion of the particles. The PanofskyWentzel theorem, 38 which is a neat way of expressing this fact, is proven in Appendix C. When the Panofsky-Wentzel theorem is applied to the coherent instabilities, one obtains the Nassibian-Sacherer relation, 39 which relates the tranverse impedance to a generalized longitudinal impedance. The relation is discussed in Appendix $D$.

\section{TRANSVERSE IMPEDANCE}

We are interested in the transverse components of the Lorentz force

$$
\overrightarrow{\mathrm{F}}=\mathbf{e}(\overrightarrow{\mathscr{E}}+\vec{B} c \times \vec{B}) \text {. }
$$

We concentrate our discussion on $F_{y}$, the $y$-component of $\vec{F}$; the discussion of $F_{x}$ is analogous.

The force field $F_{y}(\theta, t)$ is induced by various multipole components of the beam current through their interaction with the environment. The field $F_{y}$ so generated may in turn excite the multipole components of the current. This "feedback loop" provides the mechanism for the transverse coherent instability of the beam.

If the transverse dimension of the beam is small, the dominant source of $F_{y}$ is the $y$-component of the beam dipole density; we therefore ignore all other sources. The dipole density at a given moment $t$ and at an angular position $\phi=\theta-\omega_{0} t$ relative to the posi$t$ ion of the reference particle is defined as 


$$
D(\phi, t)=\langle y(\phi, t)\rangle \rho(\phi, t),
$$

where $\langle y(\phi, t)\rangle$ is the average $y$-displacement from the nominal orbit of the particles located at position $\phi$, and $\rho(\phi, t)$ is the line density normalized to $1, \int^{2 \pi} \mathrm{d} \phi \rho(\phi, t)=1$. The dipole density at time $t$ and at position $\theta$ relative to the ring is, of course, $D\left(\theta-\omega_{0} t, t\right)$.

The force field $F_{y}(\theta, t)$ generated by $D$ can in genera 1 be written as

$$
F_{y}(\theta, t)=I_{a v} \int_{0}^{2 \pi} d \theta^{\prime} \int_{-\infty}^{\infty} d t^{\prime} G\left(\theta, \theta^{\prime}, t-t^{\prime}\right) D\left(\theta^{\prime}-\omega_{0} t^{\prime}, t^{\prime}\right) .
$$

The $t-t$ ' dependence of the Green's function $G$ follows from the invariance of the dynamics under the translation in time. Since the source of the impedance may be localized objects around the ring -pickup's, cavities etc. -- the Green's function depends on $\theta$ and $\theta$ ' separately. However, we use a smooth approximation (cf. footnote following Eq. 3-11) to write

$$
G\left(\theta, \theta^{\prime}, t-t^{\prime}\right)=G\left(\theta-\theta^{\prime}, t-t^{\prime}\right) \text {. }
$$

Eq. (13-3) thus becomes

$$
F_{y}(\theta, t)=I_{a v} \cdot \int_{0}^{2 \pi} d \theta^{\prime} \int_{-\infty}^{\infty} d t^{\prime} G\left(\theta-\theta^{\prime}, t-t^{\prime}\right) D\left(\theta^{\prime}-\omega_{0} t^{\prime}, t^{+}\right) \text {. }
$$

The transverse impedance $z_{n}^{y}(\omega)$ is conventionally defined ${ }^{3}$ by

$$
G(\theta, t)=i \frac{e \omega_{0}}{4 \pi^{2} c} \sum_{n=-\infty}^{\infty} \int_{-\infty}^{\infty} d \omega z_{n}^{y}(\omega) e^{i n \theta-i \omega t} .
$$

In terms of the Fourier components of $D$ given by

$$
D(\phi, t)=\sum_{n=-\infty}^{\infty} \int_{-\infty}^{\infty} d \Omega D_{n}(\Omega) e^{i n \phi-i \Omega t},
$$

Eq. (13-5) becomes

$$
F_{y}(\theta, t)=i \frac{e \omega_{0}}{c} I_{a v} \sum_{n} \int d \Omega z_{n}^{y}\left(n \omega_{0}+\Omega\right) D_{n}(\Omega) e^{i n \phi-i \Omega t} .
$$

We discuss now the constraint imposed by the causality condition
on $z_{n}(\omega)$. The causality condition is

$$
G(\theta, t)=0 \text { if } t<0 .
$$

From Eqs. (13-6) and (13-9), 


$$
z_{n}^{y}(\omega)=-i \frac{c}{e \omega_{0}} \int_{0}^{2 \pi} d \theta \int_{0}^{\infty} d t G(\theta, t) e^{-i n \theta+i \omega t} .
$$

The function $z_{n}^{y}(w)$ can be analytically continued to the upper half of the complex ${ }^{n} \omega-p l a n e$ through this equation. It follows, then, from Eq. $(13-10)$ that

$$
\mathrm{z}_{\mathrm{n}}^{\mathrm{y}}(\omega) *=-\mathrm{z}_{-\mathrm{n}}^{\mathrm{y}}(-\omega *)
$$

In terms of the real (resistive) and the imaginary (reactive) part of $\mathrm{z}^{\mathrm{y}}$ defined by

$$
z_{n}^{y}(\omega)=\mathscr{R}_{n}^{y}(\omega)+i x_{n}^{y}(\omega)
$$

Eq. $(13-11)$ can be written as

$$
\mathscr{R}_{\mathbf{n}}^{\mathrm{y}}(-\omega *)=-\mathscr{R}_{\mathbf{n}}^{\mathrm{y}}(\omega),
$$

and

$$
x_{n}^{y}(-\omega *)=x_{n}^{y}(\omega)
$$

The positivity of $\mathscr{R}_{n}(\omega)$ is discussed in Appendix $D$.

\section{COASTING BEAM TRANSVERSE INSTABILITY}

\subsection{Equation of Motion and Dispersion Relation}

The angular revolytion frequency of a particle with fractional momentum deviation $\delta$ is $\theta=w_{0}(1-n \delta)$. Therefore, the longitudinal position of the $j$-th particle relative to the reference particle can be written as

$$
\phi_{j}=\phi_{o j}-\omega_{o} n \delta \mathrm{j} .
$$

We ignore the effects that the longitudinal perturbation on the beam may have on the transverse motion of the particle, and take the y-displacement of the particle to be a function of its longitudinal position, $\delta$ and time $t$; that is,

$$
y_{j}(t)=y\left(\phi_{j}, \delta_{j}, t\right)=y\left(\phi_{o j}-w_{o} n \delta_{j} t, \delta_{j}, t\right) .
$$

The equation of motion is

$$
\frac{d^{2}}{d t^{2}} y_{j}(t)+\omega_{y}^{2} y_{j}=\frac{1}{m \gamma_{0}} F_{y}(\theta, t)
$$

where the force $F_{y}$ should be evaluated at the position of the particle 


$$
\theta=\theta_{j}(t)=w_{0} t+\phi_{j}=w_{0} t+\phi_{0 j}-w_{0} \eta \delta_{j} t,
$$

and the angular betatron frequency $\omega_{y}$ is given by

$$
\omega_{\mathrm{y}}^{2}=Q_{\mathrm{yo}}^{2}(1+\xi \delta)^{2} \xi^{2}=\omega_{y o}^{2}(1-\pi \delta)^{2}(1+\xi \delta)^{2}
$$

where $Q_{y o}$ is the nominal $y$-tune of the ring, $\omega_{y o}=Q_{y_{0}} \omega_{0}$, and $\xi$ is the y-chromaticity.

Let us drop the subscript $j$ from now on.

From Eqs. (13-8) and (14-4)

$F_{y}(\theta, t)=i \frac{e_{0}^{\omega}}{c} I_{a v} \sum_{n} \int d \Omega z_{n}^{y}\left(n w_{0}+\Omega\right) D_{n}(\Omega) e^{i n\left(\phi_{0}-\omega o n \delta t\right)-i \Omega t} \cdot(14-6)$

Using the Fourier component of $y$ defined by

$$
y(\phi, \delta, t)=\sum_{n} \int_{d} \Omega_{n}(\delta, \Omega) e^{i n \phi-i \Omega t}
$$

Eq. (14-3) can be written as

$-\left(\Omega+n \omega_{0} n \delta\right)^{2} y_{n}(\delta, \Omega)+\omega_{y}^{2} y_{n}=i \frac{e e_{0}^{\omega}}{E_{0}} I_{a v} D_{n}(\Omega) z_{n}^{y}\left(n w_{0}+\Omega\right)$.

We now use Eq. $(13-2)$ to eliminate $y_{n}$ and $D_{n}$ from this expression.

For a coasting beam,

therefore,

$$
\rho(\phi, t)=1 / 2 \pi
$$

$$
D(\phi, t)=\langle y(\phi, t)\rangle / 2 \pi \text {. }
$$

Noting that

$$
\langle y(\phi, t)\rangle=\int d \delta_{y}(\phi, \delta, t) g(\delta),
$$

where $g(\delta)$ is the distribution function in $\delta$ normalized to 1 , $\int d \delta g(\delta)=1$, we have

$$
\mathrm{D}_{\mathrm{n}}(\Omega)=\frac{1}{2 \pi} \int_{-\infty}^{\infty} \mathrm{d} \delta \mathrm{y}_{\mathrm{n}}(\delta, \Omega) \mathrm{g}(\delta)
$$

This equation together with (14-8) yields

$$
1=-i \frac{e c \omega_{0}}{2 \pi E_{0}} I_{a v} z_{n}^{y}\left(n \omega_{0}+\Omega\right) \int_{-\infty}^{\infty} d \delta \frac{g(\delta)}{\left(\Omega+n \omega_{0} \eta \delta\right)^{2}-\omega_{y}^{2}}
$$

This is the dispersion relation for the coasting beam transverse instability. The solution $\Omega$ of Eq. (14-13) is the coherent frequency of the n-th mode, and the dipole density corresponding to this coherent mode is $D(\phi, t)=e^{i n \phi-i \Omega t}$. 
Suppose $\Omega$ is the solution of Eq. (14-13) with positive $n$; then from the symetry property $(13-11)$ of $z^{y}, \Omega^{*}$ is the solution of the same equation with $n$ replaced by $-n$. In other words, it is sufficient for us to solve Eq. (14-13) for positive $n$. Note that $\Omega$ and $\Omega$ have the same imaginary part; therefore, if mode $n$ with coherent frequency $\Omega$ is stable or unstable, the same will be true for mode $-n$ with coherent frequency $\Omega^{*}$.

We shall assume in the rest of this section that $n>0$.

14.2 Solution of Dispersion Relation (Without Landau Damping)

In this section we solve the dispersion relation (14-13) for the case of a cold beam; namely, the case whe:e

$$
g(\delta)=\delta(\delta) \text {. }
$$

From Eqs. (14-5) and (14-14), the dispersion relation (14-13) becomes

$$
\Omega^{2}=Q_{y o}^{2} \omega_{0}^{2}-i \frac{e c \omega_{0}}{2 \pi E_{0}} I_{a v} z_{n}^{y}\left(n \omega_{0}+\Omega\right)
$$

To first order in $\mathrm{z}^{\mathrm{y}}$, the two solutions of the dispersion relation (14-15) are

and

$$
\Omega_{+}=Q_{y o} \omega_{0}-i \frac{e c}{4 \pi} Q_{y o} E_{0} I a v z_{n}^{y}\left(\left(n+Q_{y o}\right) \omega_{0}\right)
$$

$$
\Omega_{-}=-Q_{y o} \omega_{0}+i \frac{e c}{4 \pi} Q_{y o} E_{0} I_{a v} z_{n}^{y}\left(\left(n-Q_{y o}\right) w_{0}\right) .
$$

To zero-th order in $\mathrm{z}^{\mathrm{y}}$, the dipole density corresponding to these two coherent modes is

$$
D_{ \pm}(\phi, t)=e^{i n \phi \mp i Q_{y_{0}} \omega_{0}}=e^{i\left(n \theta-\left(n \pm Q_{y_{0}}\right) \omega_{0} t\right)} .
$$

This equation describes waves of coherent betatron oscillation with angular phase velocities with respect to the ring given by

$$
\omega_{0} \pm \frac{Q_{y o}}{n} \text {. }
$$

We see that the phase velocity of the coherent wave corresponding to Eq. $(14-16)$ is greater than the beam velocity, while the phase velocity corresponding to $(14-17)$ is smaller. They are therefore called fast and slow waves respectively.

It is shown in Appendix $D$ that the real part of $z_{n} y(\omega)$ is positive for $n>0$ and negative for $n<0$. Therefore we conclude that for a cold beam the fast wave is always stable and the slow wave is always unstable. 40 


\subsection{Solution of Dispersion Relation (With Landau Damping)}

We investigate here the effect of the momentum spread on the transverse coasting beam instability. Equation (14-5) shows that a momentum spread induces spread in $\omega_{y}$ from the revolution frequency spread and the tune spread; therefore, the threshold condition will involve $\eta$ as well as $\xi_{\mathrm{y}}$.

Let us discuss Landau damping of the slow waves since the fast wave is always stable.

Mathmatically, Landau damping comes about from the vanishing of the denominator of the integrand of Eq. (14-13):

$$
\begin{aligned}
\left(\Omega+n \omega_{0} \eta \delta\right)^{2}-\omega_{y}^{2}= & \left(\Omega+Q_{y_{0} \omega_{0}}+\omega_{0} \delta\left\{n \eta+Q_{y_{0}}(\xi-\eta)\right\}\right) \\
& \times\left(\Omega-Q_{y_{0}} \omega_{0}+\omega_{0} \delta\left\{n \eta-Q_{y o}(\xi-\eta)\right\}\right) .
\end{aligned}
$$

For the slow wave, $\Omega \cong-Q_{y_{0}} \omega_{0}$, hence we approximate Eq. $(14-19)$ as

$$
\left(\Omega+w_{0} \eta \delta\right)^{2}-\omega_{y}=-2 Q_{y_{0}} \omega_{0}\left(\Omega+Q_{y_{0}} \omega_{0}+\omega_{0} \delta\left\{n \eta+Q_{y o}(\xi-\eta)\right\}\right) \cdot(14-20)
$$

The dispersion relation (14-13) becomes

$$
\begin{aligned}
1 & =i \frac{c}{4 \pi E_{0} Q_{y o}} e I_{a v} z^{y}\left(\left(n-Q_{y o}\right) w_{0}\right) \\
& \times \int_{-\infty}^{\infty} d \delta \frac{g(\delta)}{\left.\Omega+Q_{y o} \omega_{0}+w_{o} \delta\left(n-Q_{y o}\right) \pi+Q_{y o} \xi\right)} .
\end{aligned}
$$

Let us now specialize to the case of a Gaussian momentum distribution

$$
g(\delta)=\frac{1}{\sqrt{2 \pi \sigma_{\delta}}} \exp \left(-\delta^{2} / 2 \sigma_{\delta}^{2}\right)
$$

From the following identity valid for $\operatorname{Im}(\Omega)>0$,

we have

$$
\frac{1}{\Omega+x}=-i \int_{0}^{\infty} d \tau e^{i \tau(\Omega+x)},
$$

$$
\int_{-\infty}^{\infty} \mathrm{d} \delta \frac{g(\delta)}{\Omega+\omega_{y 0}+\delta \cdot x}=-i \frac{1}{\sigma_{\delta}|x|} \sqrt{\frac{\pi}{2}} h_{T}\left[\frac{\Omega+\omega_{y 0}}{T x \mid \sigma_{\delta}}\right] \text {, }
$$

with

$$
h_{T}(y)=\sqrt{\frac{2}{\pi}} \int_{0}^{\infty} d \tau e^{i \tau y-\tau^{2} / 2}, \quad \operatorname{Im}(y)>0 .
$$

Therefore, an equivalent expression of (14-21) is 


$$
\begin{aligned}
& 1=\frac{c}{4 \sqrt{\left.2 \pi \varepsilon_{0} Q_{y o} \omega_{0} \sigma_{\delta} \mid\left(n-Q_{y o}\right) n+Q_{y o} \xi\right)}} \operatorname{eI} z^{y} z^{y}\left(\left(n-Q_{y o}\right) w_{0}\right) \\
& \times h_{T}\left[\frac{\Omega+\omega_{y o}}{T\left(n-Q_{y o}\right) n+Q_{y o} \xi \mid \omega_{0} \sigma_{\delta}}\right] \text {. }
\end{aligned}
$$

Note that the denominator of the argument of $h_{T}$ above is the r.m.s. value of the total frequency spread.

Observe from Eq. (14-25) that

$$
\left\{\begin{array}{l}
h_{T}(0)=1, \\
\left|h_{T}(y)\right|<1, \text { if } \operatorname{Im}(y)>0, \text { or if } \operatorname{Im}(y)=0 \text { and } \operatorname{Re}(y) \neq 0 .
\end{array}\right.
$$

We deduce from Eqg. (14-26) and (14-27) that a sufficient condition for the stability of the beam or, equivalently, for the absence of a solution $\Omega$ of $(14-26)$ with $\operatorname{Im}(\Omega)>0$ is

$1>\frac{\operatorname{ceI} I_{a v}}{4 \sqrt{2 \pi E} \omega_{0} \sigma_{\text {yo }} \sigma_{\delta}\left|\left(n-Q_{y o}\right) \pi+Q_{y o} \xi\right|}\left|z^{y}\left(\left(n-Q_{y o}\right) \omega_{0}\right)\right|$.

Exercise: Show that $(14-26)$ reduces to $(14-17)$ in the limit of

$$
\frac{\operatorname{Im}(\Omega)}{T\left(n-Q_{y 0}\right) n+Q_{y o} \xi \mid \omega_{0} \sigma_{\delta}} \gg 1 \text {. }
$$

\section{BUNCHED BEAM TRANSVERSE MICROWAVE INSTABILITY}

We study here the transverse coherent instability under the following conditions:

(i) Wavelength of perturbation « bunch length.

(ii) Growth rate of instability $\gg$ synchrotron frequency $\omega_{s}$ and the revolution frequency spread.

We take the condition ( $i i$ ) to imply that we can set $\omega_{s}=0$, and that we can ignore the bunch shape distortion due to filamentation'. However, Landau damping due to revolution frequency spread will be fully taken into account. In section 19, we study the same limits with finite $\omega_{s}$ and find there that the conclusions reached here remain unchanged.

\subsection{Equation of Motion and Dispersion Relation}

If $\omega_{s}=0$, the discussion of the equation of motion for a bunched beam is similar to that for a coasting beam. Equations $(14-1)$ to (14-8) remain valid, but Eqs. (14-9) to (14-13) need to be modified so that the bunched beam structure can be properly taken into account. 
The bunched beam structure introduces coupling among the revolution modes.

Ignoring filamentation, we write the line density $\lambda$ as a function of $\phi$ and $\delta$. We further assume $\lambda(\phi, \delta)$ to be factorized,

$$
\lambda(\phi, \delta)=\lambda(\phi)_{g}(\delta)
$$

with $\lambda(\phi)$ and $g(\delta)$ both normalized to 1 .

We can now write the dipole density as

$$
D(\phi, t)=\lambda(\phi) \int_{0} \delta y(\phi, \delta, t) g(\delta)
$$

This equation relates the Fourier components of $D$ and $y$ as follows:

$$
\therefore \quad D_{n}(\Omega)=\sum_{m=-\infty}^{\infty} \lambda_{n-m} \int d \delta g(\delta) y_{m}(\delta, \Omega)
$$

where

$$
\lambda(\phi)=\sum_{n} \lambda_{n} e^{i n \phi}
$$

and $D_{n}$ and $y_{n}$ are given by Eqs. (13-7) and (14-7) respectively.

From Eqs. (15-3) and $(14-8)$, we obtain the dispersion relation

$D_{m}=-i \frac{e c \omega_{0}}{E_{0}} I_{a v} \sum_{n=-\infty}^{\infty} \lambda_{m-n} z_{n}^{y}\left(n \omega_{0}+\Omega\right) D_{n} \cdot \frac{d \delta g(\delta)}{\left(\Omega+\pi \omega_{0} \eta \delta\right)^{2}-\omega_{y}^{2}}$.

The major difference between Eqs. (14-13) and (15-5a) is the coupling among the different revolution modes for the bunched beam case.

If we keep only the slow wave component, Eq. (15-5a) becomes

$$
\begin{aligned}
& D_{m}=+i \frac{c \omega_{o}}{2 E_{0} Q_{y o}} \text { eI } \sum_{n v} \lambda_{m-n} z^{y}\left(n \omega_{0}+S \Omega\right) D_{n} \\
& \times \int \frac{d \delta g(\delta)}{\left.\Omega+\omega_{y_{0}}+w_{0} j\left(n-Q_{\text {yo }}\right) \pi+Q_{y_{0}} \xi\right)} \cdot
\end{aligned}
$$

Exercise: Show that $(15-5 a)$ reduces to $(14-13)$ if $\lambda(\phi)=1 / 2 \pi$.

In the following two sections, we explore the physical contents of Eq. (15-5a) by solving it for some impedance function $z_{n}{ }^{y}$.

\subsection{Instability Due to a High q Impedance}

Suppose that the source of the ring impedance consists of a single resonance with its q-factor so high that to a good approximation 


$$
z_{n}^{y}\left(n w_{0}+\Omega\right)=z_{n_{0}}^{y} \delta_{n, n_{0}}-z_{n_{0}}^{y *} \delta_{n}-n_{0} .
$$

Substituting this into Eq. $(1 j-5)$, we obtain

$$
\begin{aligned}
D_{m}= & -i \frac{e c w o}{E_{0}} I_{a v}\left(D_{n_{0}} \lambda_{m-n_{0}} z_{n_{0}^{y}}^{y} d \delta \frac{g(\delta)}{\left(\Omega+n_{0} \omega_{0} n \delta\right)^{2}-\omega_{y}^{2}}\right. \\
& -D_{-n_{0}} \lambda_{m+n_{0}} z_{n_{0}}^{* y} \int d \delta \frac{g(\delta)}{\left(\Omega-n_{0} \omega_{0} n \delta\right)^{2}-\omega_{y}^{2}},
\end{aligned}
$$

From Eq. $(15-4)$,

$$
\left\{\begin{array}{l}
\lambda_{n} \ll 1 \quad \text { if } \quad|n| \sigma_{\phi} \gg 1 \\
\lambda_{0}=1 / 2 \pi
\end{array}\right.
$$

where $\sigma_{\phi}$ is the r.m.s. bunch length in units of radians. Also as sumption ( $i$ ) above can be restated as

$$
\sigma_{\phi} \gg 1 / n_{0} \text {. }
$$

Therefore, for a given $m$, at least one of $\lambda_{m-n_{0}}$ and $\lambda_{m+n_{0}}$ is negligible. Thus, the modes with positive and negative $\mathrm{n}$ decouple. Take $m=n_{0}$ in Eq. $(15-7)$; the result is

$$
I=-i \frac{e d w_{0}}{2 \pi \varepsilon_{0}} I_{a v} z_{n_{0}}^{y} \int d \delta \frac{g(\delta)}{\left(\Omega+n_{0} \omega_{0} \eta \delta\right)^{2}-\omega_{y}^{2}} .
$$

Comparing this with Eq. (14-13), we conclude that for a very high q and high frequency impedance, the coherent frequency of a bunched beam is identical to that of a coasting beam with the same average current. The corresponding eigenvector in $\phi$-space is, Erom Eqs. $\frac{(15-7)}{15}$ $(15-10)$,

$$
\begin{aligned}
D(\phi, t) & =\sum_{\mathbf{m}} D_{m} e^{i m \phi-i \Omega t}=\sum_{m} \lambda_{m-n_{0}} e^{i m \phi-i \Omega t} \\
& =\lambda(\phi) e^{i n_{0} \phi-i \Omega t}
\end{aligned}
$$

up to a factor of constant. The meaning of this equation is that the cavity excites a coasting-beam-like transverse wave, and this wave is modulated by the bunch shape function $\lambda(\phi)$ so that the dipole density does, not extend outgide the region of the bunch. 
15.3 High Frequency Instability Due to a Broad Band Impedance

This case is, for a historical reason, known as the transverse microwave instability.

We assume that

$$
z_{\mathfrak{n}}^{y} \simeq z_{n_{0}}^{y} \quad \text { for } \quad\left|n-n_{0}\right|<\Delta
$$

where $\Delta$ is of the order of the inverse range of the transverse wakefield, and $n_{0} \gg \Delta \gg 1 . / \sigma_{\phi}$.

Let us find the approximate solution of the dispersion relation (15-5a) for which $D_{n}$ is negligibly small outside the range given in Eq. (15-12). Then, (15-5a) can be approximated as

$D_{m}=-i \frac{c \omega_{o}}{E_{0}} e I_{a v} z_{n_{0}}^{y} \int d \delta \frac{g i \delta)}{\left(\Omega+n \omega_{0} n \delta\right)^{2}-\omega_{y}^{2}} \sum_{n=n_{0}-\Delta}^{n_{0}+\Delta} \lambda_{m-n} D_{n}$.

Denote by $k$ the eigenvalue of the matrix $\lambda_{m-n}$ :

$$
\mathrm{KD}_{\mathrm{m}}=2 \pi \sum_{\mathrm{n}=\mathrm{n}_{0}-\Delta}^{\mathrm{n}_{\mathrm{O}}+\Delta} \lambda_{\mathrm{m}-\mathrm{n}} \mathrm{D}_{\mathrm{n}} .
$$

The coherent frequency $\Omega$ will then be determined by

$$
1=-i k \frac{c \omega_{o}}{2 \pi E_{o}} e I a_{n_{0}}^{y} \int d \delta \frac{d \delta g(\delta)}{\left(\Omega+n \omega_{c} n \delta\right)^{2}-\omega_{y}^{2}} .
$$

It remains for us to find $K$ by solving Eq. (15-14). Since $\lambda_{\text {m-n }}$ is sharply peaked at m $=n$ with peak width $s 1 / \sigma_{\phi} \ll \Delta$, we expect that the eigenvalue $K$ does not depend strongly on the cutoff value of $\Delta$. Therefore, it should be closely approximatd by

$$
K D_{m}=2 \pi \sum_{n=-\infty}^{\infty} \lambda_{m-n} D_{n}
$$

The eigenvector of Eq. $(15-16)$ is

$$
D_{n}=e^{-i \Phi n}
$$

with the cnrresponding eigenvalue

$$
\kappa(\Phi)=2 \pi \sum_{n=-\infty}^{\infty} \lambda_{n} e^{i \Phi n}=2 \pi \lambda(\Phi),
$$


where $\Phi, 0 \leq \Phi<2 \pi$, is a parameter which labels different eigensolutiōns.

Note that Eq. $(15-15)$ is the same as the coasting beam dispersion relation (14-13) with $I_{a v}$ replaced by

$$
K(\Phi) I_{\mathrm{av}}=2 \pi \lambda(\Phi) I_{\mathrm{av}}=\mathrm{e}^{\pi} \mathrm{w} \mathrm{o}_{\mathrm{o}} \lambda(\Phi)
$$

This is the local current at position $\phi=\Phi$ in the beam.

To gain some insight into the nature of the dipole density, let us take as an approximation to the eigenvector of Eq. (15-14),

$$
D_{n}(\Phi)=\left\{\begin{array}{lll}
e^{-i n \Phi} & \text { for } & \left|\mathbf{n}-n_{0}\right| \leq \Delta, \\
0 & \text { for } & \left|\mathbf{n}-n_{0}\right|>\Delta .
\end{array}\right.
$$

The dipole density is

$$
\begin{aligned}
D(\phi, t) & =\sum_{n=n_{0}-\Delta}^{n_{0}+\Delta} e^{i n(\phi-\Phi)-i \Omega t} \\
& =e^{i n_{0}(\phi-\Phi)} \frac{\sin \left(\left(\Delta+\frac{1}{2}\right)(\phi-\Phi)\right)}{\sin ((\phi-\phi) / 2)} e^{-i \Omega t} .
\end{aligned}
$$

For large $\Delta, D(\phi, t)$ is sharply peaked about $\phi=\Phi$, and the peak width is of order $1 / \Delta s$ wakelength.

16. TLASOV EQUATION FOR SINGLE BUNCH TRANSVERSE COHERENT INSTABILITY

In the discussion of the bunched beam transverse instability in the preceding section, we ignored the possible effect of the longitudinal phase focusing. To take such an effect into account, the most convenient method is that of vlasov. We formulate here the vlasov equation for the single bunch transverse instability, taking into account the finiteness of the angular synchrotron frequency $\omega_{s}$. We ignore the effect of synchrotron frequency spread.

\subsection{Equation of Motion}

If we approximate the betatron motion of a particle in a strong focusing machine by a harmonic motion, the equation of y-betatron motion is

$$
\ddot{y}+\omega_{y 0}^{2}(1-\eta \delta)^{2}(1+\xi \delta)^{2} y=0,
$$

where $\xi$ is the chromaticity. We shall not include the force induced by the coherent motion until Section 16.4 .

In the following discussion, we ignore the term $0(\delta 2)$ in Eq. $(16-1)$. To this order of approximation, $(16-1)$ can be generated by the Hamiltonian 


$$
\begin{aligned}
\mathrm{B}_{0}= & \frac{1}{2}\left(\left\{\frac{c^{2}}{E_{0}} p_{y}^{2}+\omega_{y o}^{2} \frac{E_{o}}{c^{2}} y^{2}\right\}\left\{1+(\xi-\eta) \frac{\omega_{o}}{\beta_{o}^{2} E_{o}} w\right\}\right) \\
& -\frac{1}{2}\left(\bar{n} w^{2}+\omega_{s}^{2}\left(\phi-\phi_{o}\right)^{2} / \bar{\eta}\right),
\end{aligned}
$$

where

$$
\begin{aligned}
& \bar{n}=n \omega_{0}^{2} /\left(\beta_{0}^{2} E_{0}\right), \\
& \omega=\left(E-E_{0}\right) / \omega_{0}=\left(\beta_{0}^{2} E_{0} / \omega_{0}\right) \delta,
\end{aligned}
$$

and $\phi_{0}$ is the location of the center of the bunch. This Hamiltonian describes the synchrotron motion as well as the betatron motion; the canonical pairs are $\left(\phi-\phi_{0}, W\right)$ and $\left(y, P_{y}\right)$.

It is demonstrated in Appendix B, with a weak focusing machine used as example, how one obtains Eq. (16-2) fiom the basic Hamiltonian for the Lorentz force.

\subsection{Action-Angle Variables}

The action-angle variables $\left(J_{y}, \psi_{y}\right)$ and $\left(J_{s}, \psi_{s}\right)$ can be introduced by a canonical transformation generated 41 by

$$
F_{1}=-\frac{1}{2}\left(\frac{E_{0}}{c^{2}}\right) \omega_{y o} y^{2} \tan \psi_{y}-\frac{1}{2} \frac{1}{n} \omega_{s}\left(\phi-\phi_{o}\right)^{2} \tan \psi_{s} .
$$

The transformation is

$$
\left\{\begin{aligned}
y=\bar{y} \cos \psi_{y}, & p_{y}=-\frac{E_{o}}{c} \omega_{y o} \bar{y} \sin \psi_{y}, \\
\phi-\phi_{0}=\bar{\phi} \cos \psi_{s}, & w=-\frac{1}{\bar{n}} \omega_{s} \bar{\phi} \sin \phi_{s},
\end{aligned}\right.
$$

where the betatron and the synchrotron amplutudes $\bar{y}$ and $\bar{\phi}$ are, respectively, functions of $\mathrm{J}_{y}$ and $\mathrm{J}_{\mathrm{g}}$,

$$
\bar{y}=\bar{y}\left(J_{y}\right)=\sqrt{\frac{c^{2}}{E_{0}} \frac{2 J_{y}}{w_{y o}}}, \quad \text { and } \quad \bar{\phi}=\bar{\phi}\left(J_{s}\right)=\sqrt{\bar{n} \frac{2 J_{s}}{\omega_{s}}}
$$

Also,

$$
\left\{\begin{array}{l}
J_{y}=\frac{1}{2}\left(\frac{E}{c^{2}} \omega_{y o} y^{2}+\frac{c^{2}}{E_{o}} \frac{1}{\omega_{y o}} p_{y}^{2}\right) \\
J_{s}=\frac{1}{2}\left(\frac{1}{\eta} \omega_{s}\left(\phi-\phi_{o}\right)^{2}+\frac{-}{\eta} \frac{1}{\omega_{s}} w^{2}\right) .
\end{array}\right.
$$


In terms of the action-angle variables, the Hamiltonian is

$$
H_{0}=\omega_{\text {yo }} J_{y}-\omega_{s} J_{s}+H_{H T}(J, \psi),
$$

where the head-tail Hamiltonian $\mathrm{H}_{\mathrm{HT}}$ is given by

$$
H_{H T}(J, \psi)=-a Q_{y o} \omega_{s} J_{y} \bar{\phi}\left(J_{s}\right) \sin \psi_{s},
$$

with

$$
a=\xi / \pi-1 \text {. }
$$

The head-tail Hamiltonian is usually small. It describes the modulation of the betatron oscillation by the synchrotron motion, and this modulation is the source of the head-tail mode 36,37 of the transverse coherent instability.

\subsection{Rolmogorov Transformation}

It is useful, before writing the Vlasov equation, to simplify the Hamiltonian $H_{0}$ by performing a canonical perturbation known as the Kolmogorov transformation. 42,43

Let us introduce a canonical transformation $\left(J_{y}, \psi_{y} ; J_{s}, \psi_{s}\right) \rightarrow$ $\left(k_{y}, \alpha_{y} ; R_{s}, \alpha_{s}\right)$ generated by

$$
F_{2}(R, \psi)=\psi_{y} R_{y}+\psi_{s} K_{s}+s_{2}(R, \psi),
$$

with $S_{2}$ to be specified later. The transformation is

$$
\begin{array}{ll}
J_{y}=R_{y}+\partial s_{2} / \partial \psi_{y}, & \alpha_{y}=\psi_{y}+\partial s_{2} / \partial R_{y}, \\
J_{s}=R_{s}+\partial s_{2} / \partial \psi_{s}, & \alpha_{s}=\psi_{s}+\partial s_{2} / \partial k_{s} .
\end{array}
$$

Sulstituting $(16-13)$ into $(16-9)$, we write

$$
\begin{aligned}
H_{0}= & \omega_{y o} R_{y}-\omega_{s} K_{s}+\left(H_{H T}(J, \psi)-H_{H T}(K, \psi)\right) \\
& +\left\{H_{H T}(K, \psi)+\omega_{y o} \frac{\partial s_{2}}{\partial \psi_{y}}-\omega_{s} \frac{\partial s_{2}}{\partial \psi_{s}}\right\} .
\end{aligned}
$$

We now specify $s_{2}$ by setting

$$
H_{H T}(K, \psi)+\omega_{y o} \frac{\partial}{\partial \psi_{y}} s_{2}(K, \psi)-\omega_{s} \frac{\partial}{\partial \psi_{s}} s_{2}(K, \psi)=0 .
$$

A solution of this equation is

$$
S_{2}(K, \psi)=a Q_{y o} K_{y} \bar{\phi}\left(K_{s}\right) \cos \psi_{s},
$$

and (16-13) becomes 


$$
\left\{\begin{array}{l}
J_{y}=R_{y}, \\
\alpha_{y}=\psi_{y}+a Q_{y o} \bar{\phi}\left(R_{s}\right) \cos \psi_{s}, \\
J_{s}=R_{s}\left(1-a Q_{y o} \frac{R_{y}}{R_{s}} \bar{\phi}\left(R_{s}\right) \sin \psi_{s}\right), \\
\alpha_{s}=\psi_{s}+\frac{1}{2} a_{y o} \frac{R_{y}}{R_{s}} \bar{\phi}\left(R_{s}\right) \cos \psi_{s} .
\end{array}\right.
$$

Note that $\mathrm{H}_{\mathrm{HT}}(\mathrm{J}, \psi)=O\left(\mathrm{~J}_{\mathrm{y}} \sqrt{\mathrm{J}_{\mathrm{s}}}\right)$, and that, from $(16-17)$, $\mathrm{H}_{\mathrm{HT}}(\mathrm{J}, \psi)-\mathrm{H}_{\mathrm{HT}}(\mathrm{K}, \psi)=0\left(\mathrm{~J}_{\mathrm{Y}}^{2} / \sqrt{\mathrm{J}_{\mathrm{S}}}\right)$. Therefore we ignore the terms in (...) in Eq. $(16-14)$ on the ground that the ratio of the emittances, $\mathrm{J}_{\mathrm{y}} / \mathrm{J}_{s}$, is generally very small. We thus obtain

$$
\mathrm{H}_{0} \simeq w_{y_{0}} R_{y}-w_{s} R_{s} \cdot
$$

The head-tail Hamiltonian has been transformed away in (16-18); the mechanism of head-tail instability is contained now in the transformation (16-17).

The last terms of $(16-17 c)$ and $(16-17 d)$ can also be ignored because $\mathrm{K}_{\mathrm{y}} / \mathrm{R}_{\mathrm{s}} \backsim \mathrm{J}_{\mathrm{y}} / \mathrm{J}_{\mathrm{s}}$. Hence,

$$
\left\{\begin{array}{l}
J_{y}=R_{y}, \quad \alpha_{s} \simeq \psi_{s}, \quad J_{s} \simeq k_{s} \\
\alpha_{y}=\psi_{y}+2 Q_{y o} \bar{\phi}\left(J_{s}\right) \cos \psi_{s} .
\end{array}\right.
$$

From (16-18) and the Hamiltonian equation, $\alpha_{y}=\omega_{\text {yot }}$; therefore, $(16-20)$ describes the precise way the betatron phase $\psi_{y}^{y 0}$ is modulated by the synchrotron oscillation.

\subsection{Inclusion of Coherent Force}

So far in this section, the transverse force $F$ induced by the coherent motion of the beam is not included in the discussion. We now write the total Hamiltonian including the coherent effect as

where

$$
\mathrm{H}=\mathrm{H}_{\mathrm{O}}+\mathrm{U}^{\mathrm{y}}(\phi, t)
$$

$$
-\frac{\partial U^{y}}{\partial y}=F_{y}\left(\phi+w_{0} t, t\right)
$$
with $F_{y}(\theta, t)$ given by Eq. $(13-8)$. Since $F_{y}$ is independent of $y$, we
have

$$
U^{y}(\phi, t)=-y F_{y}\left(\phi+\omega_{0} t, t\right)
$$




\subsection{Vlasov Equation} is

The Vlasov equation for the phase space density $\Psi\left(R_{s}, \alpha_{s} ; R_{y}, \alpha_{y} ; t\right)$

$$
\frac{\partial \Psi}{\partial t}+(\Psi, \mathrm{H})=0
$$

where (, ) is the Poisson bracket defined by

$$
(A, B)=\frac{\partial A}{\partial \alpha_{s}} \frac{\partial B}{\partial R_{s}}-\frac{\partial A}{\partial K_{s}} \frac{\partial B}{\partial \alpha_{s}}+\frac{\partial A}{\partial \alpha_{y}} \frac{\partial B}{\partial K_{y}}-\frac{\partial A}{\partial K_{y}} \frac{\partial B}{\partial \alpha_{y}} .
$$

We adopt the normalization

$$
\int_{0}^{2 \pi} d \alpha_{s} \int_{0}^{\infty} d k_{s} \int_{0}^{2 \pi} d \alpha_{y} \int_{0}^{\infty} d k_{y} \Psi=1
$$

For the Hamiltonian given by Eq. (16-21) with (16-18), we can write Eq. $(16-24)$ as

$$
\frac{\partial \Psi}{\partial t}-\omega_{s} \frac{\partial \Psi}{\partial \alpha_{s}}+\omega_{y o} \frac{\partial \Psi}{\partial \alpha_{y}}+\left(w, \sigma^{y}\right)=0 \text {. }
$$

We solve this equation perturbatively to first order in impedance $\mathrm{z}^{\mathrm{y}}$. Recalling that $\mathrm{O}^{\mathrm{y}}$ is of $O\left(\mathrm{Z}^{\mathrm{y}}\right)$, we see that the zero-th order solution $\Psi_{0}$ of Eq. $(16-27)$ is a function of $R_{s}$ and $R_{y}$ only. Hence, we write

$$
\begin{aligned}
\Psi_{1} & =\Psi_{0}\left(R_{s}, R_{y}\right)+\Psi_{1}\left(R_{2}, \alpha_{s} ; R_{y}, \alpha_{y} ; t\right) \\
& =\Psi_{0}\left(J_{s}, J_{y}\right)+\Psi_{1}\left(J_{s}, \Psi_{s} ; J_{y}, \alpha_{y} ; t\right),
\end{aligned}
$$

where $(16-19)$ has been used, and $\Psi_{1}$ is taken to be of first order in $z^{y} . \Psi_{1}$ satisfies

$$
\frac{\partial}{\partial t} \Psi_{1}\left(J_{s}, \Psi_{s} ; J_{y}, \alpha_{y} ; t\right)-\omega_{s} \frac{\partial \Psi_{s}}{\partial \Psi_{s}}+\omega_{y o} \frac{\partial \Psi_{1}}{\partial \alpha_{y}}+\left(\Psi_{0}, v^{y}\right)=0 \text {. }
$$

Recalling that a Poisson bracket is invariant under a canonical transformation, the Poisson bracket of Eq. (16-29) can be evaluated as

$$
\begin{aligned}
\left(\Psi_{0}, U^{y}\right) & =-\frac{\partial \Psi}{\partial P_{y}} \frac{\partial U^{y}}{\partial y}-\frac{\partial \Psi}{\partial W} \frac{\partial U^{y}}{\partial \phi} \\
& =\frac{\partial \Psi_{o}}{\partial P_{y}} F_{y}-\frac{\partial \Psi}{\partial W} \frac{\partial U^{y}}{\partial \phi} .
\end{aligned}
$$

The last term above reflects the fact that the energy for the transverse coherent motion is provided by the longitudinal energy of the 
beam motion (cf. Appendix C); nevertheless, we shall drop this term. From Eqq. $(16-6)$ to $(16-8)$,

$$
\frac{\partial \Psi_{o}\left(J_{s}, J_{y}\right)}{\partial p_{y}}=-\bar{y}\left(J_{y}\right) \sin \psi_{y} \frac{\partial \Psi_{o}\left(J_{s}, J_{y}\right)}{\partial J_{y}} F_{y}
$$

therefore, Eq. (16-29) becomes

$\frac{\partial}{\partial t} \Psi_{1}-\omega_{s} \frac{\partial \Psi_{1}}{\partial \Psi_{s}}+\omega_{y 0} \frac{\partial \Psi_{1}}{\partial \alpha_{y}}=\bar{y}\left(J_{y}\right) \sin \psi_{y} \frac{\partial \Psi_{o}\left(J_{s}, J_{y}\right)}{\partial J_{y}} F_{y}\left(\phi+\omega_{o} t, t\right)$.

Since the only $\psi_{y}$ dependence of the right-hand side is through $\sin \psi_{y}$, and $\alpha_{y}$ and $\psi_{y}$ are related linearly by Eq. $(16-20)$, the transverse part of $(16-30)$ is relatively easy to solve. We set

$$
\sin \psi_{y}=\frac{1}{2 i}\left(e^{i \psi_{y}}-e^{-i \psi_{y}}\right)+\frac{i}{2} e^{-i \psi_{y}}
$$

This amounts to assuming the decoupling of the coherent modes with coherent frequencies $\Omega \simeq \omega_{\mathrm{y}}$ and $\Omega \simeq \omega_{\mathrm{y}}$ and choosing to discuss the former. Eq. (16-30) now becomes

$$
\frac{\partial}{\partial t} \Psi_{1}-w_{s} \frac{\partial \Psi_{1}}{\partial \Psi_{s}}+w_{y o} \frac{\partial \Psi_{1}}{\partial \alpha_{y}}=\frac{i}{2} e^{-i \psi_{y}} \bar{y} \frac{\partial \Psi_{0}\left(J_{s}, J_{y}\right)}{\partial J_{y}} F_{y} .
$$

The $\alpha_{y}$ part of this equation can easily be integrated, and the equation becomes

$$
-i\left(\Omega+\omega_{y o}\right) \Phi-\omega_{s} \frac{\partial \Phi}{\partial \psi_{s}}=\frac{i}{2} e^{i a Q_{y o} \bar{\phi} \cos \psi_{s}} \frac{\partial \Psi_{o}}{\partial J_{y}} f_{y},
$$

where

$$
\Psi_{1}\left(J_{s}, \psi_{s} ; J_{y}, \alpha_{y} ; t\right)=e^{-i \alpha_{y}} \Phi\left(J_{s}, J_{y}, \psi_{s}\right) e^{-i \Omega t},
$$

and, from Eqs. (13-8) and $(16-6)$,

$\tilde{F}_{y}\left(J_{s}, \psi_{s}\right)=i \frac{e^{\omega_{0}}}{c} I_{a v} \sum_{n} D_{n}(\Omega) Z_{n}^{y}\left(n \omega_{0}+\Omega\right) \exp \left(i n \bar{\phi}\left(J_{s}\right) \cos \psi_{s}\right)$,

where we have set the bunch-center location $\phi_{0}=0$, and $D_{n}$ will be related to $\Phi$ later.

We close this subsection by a few comments on $\Psi_{0}$. Recalling that the phase space volume is invariant under a canonical transformation, we have from Eq. (16-26)

Now, defining

$$
\int \mathrm{dJ}_{\mathrm{s}} \mathrm{d} \mathrm{J}_{\mathrm{y}} \Psi_{\mathrm{o}}\left(\mathrm{J}_{\mathrm{s}}, \mathrm{J}_{\mathrm{y}}\right)=\frac{1}{4 \pi^{2}} \text {. }
$$

$$
\Psi_{0}\left(J_{s}\right)=2 \pi \int d J_{y} \Psi_{0}\left(J_{s}, J_{y}\right)
$$


so that

$$
\operatorname{ldJ}_{s} \Psi_{0}\left(J_{s}\right)=\frac{1}{2 \pi}
$$

we obtain from Eq. (16-7) and an integration by parts

$$
\int_{0}^{\infty} d J_{y} \bar{y}^{2} \frac{\partial \Psi_{0}\left(J_{s}, J_{y}\right)}{\partial J_{y}}=-\frac{c^{2}}{\pi E_{0} w_{0}} \Psi_{o}\left(J_{s}\right) .
$$

16.6 Secular Equation

Here we transform the Vlasov equation (16-32) into a secular equation with $D_{n}, n= \pm 1, \pm 2, \ldots$, as the eigenvector.

First, note from (A-1) of Appendix A that (16-32) is equivalent to

$$
\begin{aligned}
\Phi\left(J_{s}, J_{y^{\prime}}, \psi_{s}\right)= & \frac{i}{2 \omega_{s}} \frac{1}{1-e^{i 2 \pi Q}} \int_{0}^{2 \pi} d \psi^{\prime} e^{i Q \psi^{\prime}} e^{i Q_{y} \phi \cos \left(\psi_{s}+\psi^{\prime}\right)} \frac{\partial \Psi_{y}\left(J_{s}, J_{y}\right)}{\partial J_{y}} \\
& \times E_{y}\left(J_{s}, \psi_{s}+\psi^{\prime}\right),
\end{aligned}
$$

where

$$
Q=\left(\Omega+\omega_{\text {yo }}\right) / \omega_{s} .
$$

Next, let us derive the relationship between $D_{\text {and }} \Phi$. The dipole density $D(\phi, t)$ is, from Eqs. $(13-2)$ and $(16-5)$, related to $\Psi$ by

$$
\begin{aligned}
D(\phi, t) & =\int d R_{s} d \alpha_{s} d R_{y} d \alpha_{y} \delta\left(\phi-\bar{\phi} \cos \phi_{s}\right) y \Psi\left(R_{s}, \alpha_{s} ; R_{y}, \alpha_{y} ; t\right) \\
& =\int d J_{s} d \psi_{s} d J_{y} d \psi_{y} \delta\left(\phi-\bar{\phi} \cos \phi_{s}\right) y \Psi\left(J_{s}, \psi_{s} ; J y, \alpha_{y} ; t\right) .
\end{aligned}
$$

We observe that the unperturbed part, $\Psi_{o}\left(J_{g}, J_{y}\right)$, of $\Psi$ does not contribute to the above integral since $\Psi_{0}$ is an even function of $y_{\text {. Hence }}$ we obtain the following expression for the Fourier component of the dipole density by using Eqs. $(13-7),(16-33)$, and $(16-41)$ :

$D_{n}(\Omega)=\frac{1}{2} \int_{d J_{s}} d \psi_{s} d J_{y} e^{-i\left(n+a Q_{y o}\right) \Phi\left(J_{s}\right) \cos \psi_{s}\left(J_{y}\right) \Phi\left(J_{s}, J_{y}, \psi_{s}\right)}$.

Now, substituting Eq. (16-39) into (16-42) and noting that the $J_{y}$ integration in the resulting equation can be perforned with the help of Eq. (16-38), we obtain

$$
D_{m}=\sum_{n=-\infty}^{\infty} T_{m n} D_{n}
$$

with 


$$
\begin{gathered}
T_{m n}=\frac{e I_{a v} c}{4 \pi E \omega_{0} \omega_{s} Q_{y o}} \frac{z_{n}^{y}\left(n \omega_{0}+\Omega\right)}{1-e^{i 2 \pi Q}} \int_{0}^{\infty} d J_{s} \int_{0}^{2 \pi} d \psi^{\prime} d \psi_{s} \Psi_{0}\left(J_{s}\right) e^{i Q \psi^{\prime}} \\
\quad \times e^{i \bar{\phi}\left(\tilde{n} \cos \left(\psi_{s}+\psi^{\prime}\right)-\tilde{m} \cos \psi_{s}\right)}
\end{gathered}
$$

where

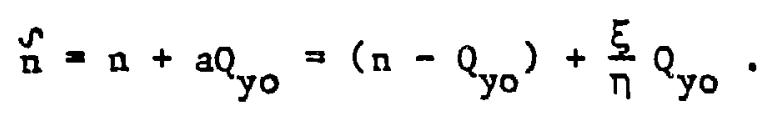

We note that all the variables related to the transverse dimension have been integrated out. The $\psi_{s}$ integration above can also be done (see Eq. $(\mathrm{A}-2))$. The result is

$$
\begin{aligned}
T_{m n}= & \frac{e I_{a v} c}{2 E_{0} \omega_{s} Q_{y o}} \frac{z_{n}^{y}\left(n \omega_{0}+\Omega\right)}{1-e^{i 2 \pi Q}} \int_{0}^{\infty} d J_{s} \int_{0}^{2 \pi} d \psi e^{i Q \psi} \\
& \times \Psi_{0}\left(J_{s}\right) J_{0}\left[\sqrt{\hat{f}^{2}+f^{2}-2 n f \cos \psi} \bar{\phi}\left(J_{s}\right)\right] .
\end{aligned}
$$

For the rest of Part II, we restrict our discussion to the case where the longitudinal distribution of the bunch in Gaussian. Taking into account the normalization condition (16-37), we set

$$
\Psi_{0}\left(J_{s}\right)=\frac{1}{2 \pi} \frac{\bar{\eta}}{\omega_{s} \sigma_{\phi}^{2}} e^{-\bar{\phi}^{2} / 2 \sigma_{\phi}^{2}},
$$

where $\sigma_{\phi}$ in the $\mathrm{r} . \mathrm{m.s}$. bunch length in units of radians. The $\mathrm{J}_{\mathrm{s}}$ integration in Eq. $(16-46)$ can now be performed. Using Eq. $(A-4)$, we obtain

$$
\begin{aligned}
T_{\mathrm{mm}}= & \frac{\operatorname{ceI}{ }_{a v}}{4 \pi Q_{y o} \omega_{s} E_{0}} \frac{z_{n}^{y}\left(n \omega_{0}+\Omega\right)}{1-e^{i 2 \pi Q}} \int_{0}^{2 \pi} d \psi e^{i Q \psi} \\
& \times e^{-\left(K^{2}+f^{2}-2 f f \cos \psi\right) \sigma_{\phi}^{2} / 2 .}
\end{aligned}
$$

We calculate for later use the line density $\lambda(\phi)$ and the momentum discribution function $g(\delta)$ curresponding to Eq. $(16-47)$. From the canonical invariance of the phase space volume,

$\Psi_{0}\left(J_{s}\right) d J_{s} d \Psi_{s}=\Psi_{0} d \phi d W$

$$
=\frac{1}{\sqrt{2 \pi} \sigma_{\phi}} e^{-\phi^{2} / 2 \sigma_{\phi}^{2}} \frac{1}{\sqrt{2 \pi} \sigma_{W}} e^{-W^{2} / 2 \sigma_{W_{d W}}^{2}} .
$$

with 


$$
\sigma_{W}=\omega_{s} \sigma_{\phi} / \bar{n} \text {. }
$$

Equations (16-7) and (16-8) have been used in obtaining (16-49). Using Eq. $(16-4)$, we obtain

$$
\frac{i}{\sqrt{2 \pi} \sigma_{W}} e^{-W^{2} / 2 \sigma_{W}^{2}} d W=\frac{1}{\sqrt{2 \pi \sigma} \delta} e^{-\delta 2 / 2 \sigma_{\delta}^{2}} d \delta,
$$

with

$$
\sigma_{\delta}=\frac{\omega_{0}}{\beta_{0}^{2} \sigma_{W}} \sigma_{W}
$$

Therefore,

$$
\lambda(\phi)=\frac{1}{\sqrt{2 \pi \sigma_{\phi}}} e^{-\phi 2 / 2 \sigma_{\phi}^{2}}
$$

and

$$
g(\delta)=\frac{1}{\sqrt{2 \pi \sigma} \delta} e^{-\delta 2 / 2 \sigma \delta}
$$

Soth $\lambda(\phi)$ and $g(\delta)$ are normalized to 1 , and the Fourier component of $\lambda(\phi)$ is

$$
\lambda_{n}=\frac{1}{2 \pi} e^{-n^{2} \sigma_{\phi}^{2} / 2}
$$

We remark that, from Eqs. $(15-49 b)$ and $(15-50 b)$ and the definition (16-3) for $\bar{n}$, we have

$$
\sigma_{\delta}=\omega_{s} \sigma_{\phi} /\left(\pi \omega_{0}\right)
$$

We close this section with a short summary. If one ignores the force induced by the collective motion of the beam, the motion of a particle in the beam is described within a smooth approximation by the Hamiltonian (16-2). Tais Hamiltonian couples the transverse and the longitudinal motions. The dynamics of this Hamiltonian is solved to the lowest order of s-y coupling by a Rolmogorov transformation which leads to an uncoupled Hamiltonian (16-18), and the solution is described by Eqs. $(\sqrt{6-19})$ and $(16-20)$. We then establish the Vlasov equation (16-24) including the collective force. In the later part of this section, it is demonstrated that the linearized vlasov equation $(16-31)$ is equivalent to the secular equation $(16-43)$ with the Fourier components of the dipole density as the eigenvector. By specializing to a Gaussian bunch, we finally obtained a relatively simple expression (16-48) for the matrix of the secular equation.

$$
\text { Al1 the following discussion in Part II is based on Eqs. (16-43) }
$$
and $(16-48)$. 
Exercise. In deriving the secular equation $(16-43,44)$, we did the replacement $(16-30 a)$. Derive the secular equation corresponding to the alternative replacement sin $\psi_{y}+$ $-i / 2 e^{i \phi y}$. Show that if $\Omega$ is a coherent Erequency of the new secular equation, then $-\Omega *$ is a solution of $(16-43,44)$. Note that $\operatorname{Im}(\Omega)=\operatorname{Im}(\Omega \star)$; therefore, the stability condition is not changed by the choice of the replacement.

\section{HEAD-TAIL MODE 36,37}

The integral representation $(16-48) \mathrm{f}$ the matrix element $\mathrm{T}_{\operatorname{mn}}$ can be transformed into a modified Bessel series. From Eq. (A-9), the matrix element becomes

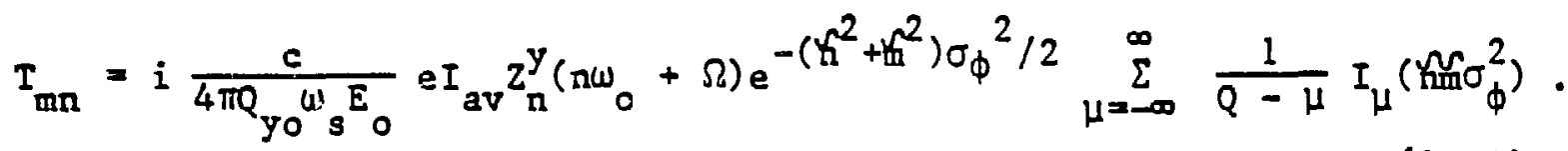

We assume in this section that

$$
\frac{c}{4 \pi Q_{y_{0} \omega_{s} E_{0}}} e I_{a v}\left|z_{n}^{y}(n \omega+\Omega)\right| \ll 1 \text {. }
$$

The condition for a coherent mode is, from Eq. (16-43), that $T_{\text {mn }}$ has 1 as one of $i$ ts eigenvalues. Under $(17-2)$, the matrix $\left(T_{\operatorname{mn}}\right)$ cannot possibly satisfy this condition unless for some integer $\mu$

$$
Q \simeq \mu \quad \text { or } \quad \Omega \simeq-\omega_{\text {yo }}+\mu \omega_{s} \text {. }
$$

The transverse coherent mode which satisfies $(17-3)$ is called the $\mu$-th head-tail mode. $\mu=0$ mode is called the rigid mode; $\mu=$ \pm 1 mode, the dipole mode; $\mu= \pm 2$ mode, the quadrupole mode; etc.

For the $\mu$-th head-tail mode, we approximate Eq. $(17-1)$ by

$$
\begin{aligned}
I_{\operatorname{mn}}= & i \frac{c}{4 \pi Q_{y_{0}{ }_{s} E_{0}}} e I_{a v} z_{n}^{y}\left(n \omega_{0}-\omega_{y o}+\omega_{s}\right) \\
& \times \frac{1}{Q-\mu} e^{\left(\kappa^{2}+\kappa^{2}\right) \sigma_{\phi}^{2} / 2} I_{\mu}\left(\sigma_{\phi}^{2}\right) .
\end{aligned}
$$

Suppose the wavelength of the perturbation is longer than the bunch length; then we can approximate the modified Bessel function by

$$
I_{\mu}\left(n \sigma_{\phi}^{2}\right) \simeq \frac{1}{|\mu| ! 2|\mu|}\left(\operatorname{mfo\sigma }_{\phi}^{2}\right)|\mu|
$$


and $\mathrm{Eq} .(17-4)$ becomes

$$
\begin{aligned}
T_{\text {mn }}= & i \frac{c e I_{a v}}{4 \pi Q_{y o} \omega_{s} E_{0}} z_{n}^{y}\left(w_{0}-\omega_{y o}+\mu_{s}\right) \frac{1}{Q-\mu} e^{-\left(\mathcal{H}^{2}+\kappa_{\phi}^{2}\right) \sigma_{\phi}^{2} / 2} \\
& \times \frac{1}{|\mu| ! 2|\mu|}\left(\operatorname{fff}_{\phi}^{2}\right)|\mu|
\end{aligned}
$$

This is a matrix of rank 1 ; hence it can easily be diagonalized. We obtain from Eqs. (16-43) and (17-5)

$$
\begin{aligned}
\left.\Omega+w_{y 0}-\psi_{s}\right)_{s}= & i \frac{c e I_{a v}}{4 \pi Q_{y o} E_{0}} \frac{1}{|\mu| ! 2|\mu|} \sum_{n=-\infty}^{\infty}\left(\sigma_{\phi}^{\mathfrak{n}}\right)^{2}|\mu| \\
& x e^{-\kappa^{2} \sigma_{\phi}^{2}} z^{y}\left(n w_{0}-\omega_{y o}+\mu w_{s}\right) .
\end{aligned}
$$

This is the expression for the coherent frequency shift of the $\mu-t h$. head-tail mode. If the imaginary part of the right-hand side is positive, this mode is unstable.

We ignored above the Landau damping of the head-tail modes. The effects of the synchrotron frequency spread on these modes are discussed in Ref. 23.

\section{TRANSVERSE STRONG COUPLING - SHORT BUNCH CASE}

We saw in Section 17 that, if the interaction between the beam and the EM fields that it induces is weak, the possible bunched beam coherent instabilities are the head-tail modes. The $\mu$-th head-tail mode, $\mu=0, \pm 1, \pm 2, \ldots$, has the coherent frequency $\Omega \simeq \omega_{\text {yo }}+\omega_{s}$, or $Q \cong \mu$. This is no longer the case if the interaction is strong. Then, the matrix $\left(\mathrm{T}_{\mathrm{mn}}(\Omega)\right)$ may have 1 as one of its eigenvalues without $Q$ being close to an integer; thus, many terms in the summation in $\mathrm{Eq}$. (17-1) may contribute with comparable strength to a coherent mode. When $Q$ is not close to an integer, $\mu$ ceases to be a good mode number to characterize an eigenmode.

We also saw that when the bunch length is short compared to the perturbing EM wavelengths, we can diagonalize the matrix (17-4) for the $\mu$-th head-tail mode by approximating it with a matrix of rank 1 . Here we generalize this method to the strong coupling case when the bunch is short. Our method consists of expanding Eq. (17-1) in a series of small parameters $f \sigma_{\phi}$ and $f \sigma_{\phi}$ and thereby approximating the o-dimensional secular equation $(16-43)$ by a new secular equation in a finite-dimensional vector space.

We recall that the transverse coherent modes are determined by the following secular equations:

$$
D_{m}=\sum_{n=-\infty}^{\infty} T_{m n}(\Omega) D_{n},
$$


with

$$
\begin{aligned}
& T_{m n}=i \chi_{n} e^{-\left(\mathcal{K}^{2}+\tilde{L}^{2}\right) \sigma_{\phi}^{2} / 2} \sum_{\mu=-\infty}^{\infty} \frac{1}{Q-\mu} I_{\mu}\left(\mathfrak{m n}_{\phi}^{2}\right), \\
& X_{n}=\frac{c}{4 \pi Q_{\text {yo }} \omega_{s} E_{0}} e I_{a v} z_{n}^{y}\left(n \omega_{0}+\Omega\right), \\
& Q=\left(\Omega+\omega_{y o}\right) / \omega_{s}, \quad \tilde{n}=n-Q_{y o}+\xi_{Q_{y o}} / \pi .
\end{aligned}
$$

Let us expand the modified Bessel functions in (18-2) in Taylor series. Then, after recombining the terms, the matrix element becomes

$$
T_{m n}=i \chi_{n} e^{-\left(\mathscr{m}^{2}+\mathscr{h}^{2}\right) \sigma_{\phi}^{2} / 2} \sum_{\ell=0}^{\infty} a_{\ell}\left(m n \sigma_{\phi}^{2}\right)^{\ell},
$$

where

$$
\begin{array}{ll}
a_{0}=\frac{1}{Q}, \quad a_{1}=\frac{Q}{Q^{2}-1}, \quad a_{2}=\frac{1}{2} \frac{Q^{2}-2}{Q\left(Q^{2}-4\right)}, \\
a_{3}=\frac{Q}{4}\left(\frac{1}{Q^{2}-1}+\frac{1}{6} \frac{1}{Q^{2}-9}\right),
\end{array}
$$

Each term in Eq. $(18-5)$ is factored into the product of a function of $m$ and a function of $n$. We use this fact to perform the following. change of base:

$$
\bar{D}_{l}=i \sum_{n=-\infty}^{\infty} x_{n} e^{-f^{2} \sigma_{\phi}^{2} / 2}\left(\tilde{n}_{\sigma_{\phi}}\right)^{l} .
$$

Then, in terms of the new basis, Eq. (18-1) becomes

$$
\begin{gathered}
\overline{\mathrm{D}}_{\ell}=\sum_{\ell^{\prime}=0}^{\infty} \overline{\mathrm{T}}_{\ell \ell^{\prime}} \overline{\mathrm{D}}_{\ell}, \\
\overline{\mathrm{T}}_{\ell \ell^{\prime}}=a_{\ell^{\prime}}, \bar{F}_{\ell+\ell^{\prime}}, \\
\mathscr{F}_{\ell}=i \sum_{\mathrm{n}=-\infty}^{\infty} x_{\mathrm{n}} \mathrm{e}^{-\hat{\hbar}^{2} \sigma_{\phi}{ }^{2}\left(\hat{\mathrm{n}}_{\phi}\right)^{\ell} .}
\end{gathered}
$$

Equation (18-8) provides a convenient starting point for treating the coherent motion of a small bunch. We assume that there exists an $n_{\max }$ such that $\chi_{n}$ is negligible if $|n|>n_{\max }$. Then, $\bar{T}_{\ell \ell}$ ' 
decreases with increasing $\ell$ and $\ell$ ' and hence (18-8) can be truncated at $l, \ell^{\prime}=\ell_{\max }$, where $\ell_{\max }$ is determined by $\max _{\max } \sigma_{\phi}$. Now $(18-8)$ becomes

$$
\bar{D}_{\ell}=\sum_{\ell^{\prime}=0}^{\ell=0} T_{\ell \ell}, \bar{D}_{\ell}, \cdot
$$

This secular equation in a finite-dimensional space can be treated in an elementary way. The coherent frequency is determined by

$$
\operatorname{det}\left(\bar{T}_{\ell \ell^{\prime}}-\delta_{\ell \ell^{\prime}}\right)=0 .
$$

We illustrate the above method by the case $\ell_{\max }=1$. Then Eq. (18-12) involves a $2 \times 2$ determinant; the equation can be written out as

$$
Q^{3}-\left(\mathscr{F}_{0}+\mathscr{F}_{2}\right) Q^{2}+\left(\mathscr{F}_{0} \mathscr{F}_{2}-1-\mathscr{F}_{1}^{2}\right) Q+\mathscr{F}_{0}=0
$$

In the weak coupling limit, $\mathscr{F}_{0}, \mathscr{F}_{1}$, and $\mathscr{F}_{2}$ are small quantities; hence Eq. $(18-13)$ can be solved perturbatively to first order in $\mathscr{F}^{r} \mathrm{~s}$. The three solutions are

$$
Q=\mathscr{F}_{C}, \quad 1+\frac{1}{2} \mathscr{F}_{1}, \quad-1+\frac{1}{2} \mathscr{F}_{1} .
$$

These solutions are identical to Eq. $(17-6)$ for $\mu=0,1$, and -1 , respectively.

For the strong coupling case, it is best to leave the solution of Eq. (18-12) to the computers.

\section{TRANSVERSE STRONG COUPLING - LONG BUNCH CASE}

We discussed in the last two sections the small bunch approximation to the secular equations $(16-43)$ : Here we discuss the opposite asymptotic limit,

$$
\mathrm{n} \sigma_{\phi} \gg 1
$$

in the case of fast blowup,

$$
\operatorname{Im}(\Omega) / \omega_{s} \gg 1 \text {. }
$$

Recall that these limits have already been treated in Section 15 by setting $\omega_{s}=0$. Here $\omega_{s}$ will be kept finite, and it will be shown that the secular equation (16-43) with matrix element (16-48) reduces to the secular equation of Section 15 in the above limits. Our present proof is valid only for the case of a Gaussian bunch.

Before going on, we also recall that, for a Gaussian bunch defined by Eq. (16-47), the normalized line density and momentum dis- 
tribution function are given, respectively, by Eqs. (16-51) and (16-52), and that the Fourier components of the line density are given by $(16-53)$.

We now find an approximate expression for the matrix element $T_{\text {mn }}$ in the limit of (19-2). In this limit, the integral in $(16-48)$ is dominated by the contribution from the integration region $\psi \simeq 0$. Therefore,

$$
\begin{aligned}
& \left.\int_{0}^{2 \pi} d \psi e^{i Q \psi} e^{-\left(\kappa^{2}+\kappa^{2}-2 \hbar f \cos \psi\right.}\right) \sigma_{\phi}^{2} / 2 \\
& \simeq e^{-(n-m)^{2} \sigma_{\phi}^{2} / 2} \int_{0}^{2 \pi} d \psi e^{i Q \psi} e^{-מ n f f \psi^{2} \sigma_{\phi}^{2} / 2} .
\end{aligned}
$$

We now take the high frequency limit,

$$
|\mathrm{n}| \sigma_{\phi}, \quad|\mathrm{m}| \sigma_{\phi} \gg 1 .
$$

Then, if $n$ and m are of opposite sign, (19-3) becomes vanishingly small because of the exponential factor in front of the integral. In other words, the fast and the slow waves decouple in the high frequency fast blowup limit. Let us consider the slow waves, i.e. $n$ and $m>0$. The upper limit of integration on the right-hand side of (19-3) can now be replaced by $\infty$ because of $(19-4)$. Thus,

$$
\begin{aligned}
\int_{0}^{2 \pi} d \psi e^{i Q \psi} & e^{-\left(\hat{h}^{2}+\kappa^{2}-2 f f \operatorname{cod} \psi\right) \sigma_{\phi}^{2} / 2} \\
& \simeq \sqrt{\frac{\pi}{2}} \frac{1}{\sqrt{f f f} \sigma_{\phi}} e^{-(n-m)^{2}} \sigma_{\phi}^{2} h_{T}\left(\frac{Q}{\sqrt{f f f} \sigma_{\phi}}\right),
\end{aligned}
$$

with

$$
h_{T}(y)=\sqrt{\frac{2}{\pi}} \int_{0}^{\infty} d x e^{i x y} e^{-x^{2} / 2} .
$$

We have already enceountered the function $h_{\mathrm{T}}$ in Section 14.

From $1 /\left(1-e^{i 2 \pi Q}\right) \rightarrow 1$ in the 1 imit of $(19-2)$, the matrix element $(16-48)$ can now be approximated by

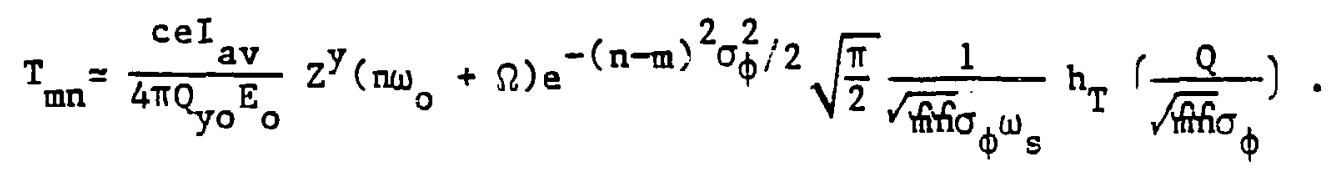


From the factor $\exp \left(-(n-m) 2 \sigma_{\phi}^{2} / 2\right)$ in this equation, we see that $T_{\operatorname{mn}}$ is vanishingly small unless $|n-m| \sigma_{\phi} \leqslant 1$. As a result, we can approximate $(19-7)$ by

$T_{m n}=\frac{\operatorname{ce} I_{a v}}{4 \pi Q_{y o} E_{0}} z_{n}^{y}\left(n \omega_{0}+\Omega\right) e^{-(n-m)^{2} \sigma_{\phi}^{2} / 2} \sqrt{\frac{\pi}{2}} \frac{1}{\underbrace{}_{n \sigma_{\phi} \omega}} h_{T}\left(\frac{Q}{n s_{\phi}}\right)$.

The error incurred in this approximation is about a factor of $1 / \mathrm{no}_{\phi}$ smaller than (19-8).

To proceed, we recall (see $(16-40))$ that

$$
\frac{Q}{\tilde{L} \sigma_{\phi}}=\frac{\Omega+\omega_{y_{0}}}{\kappa \sigma_{\phi} \omega_{s}} \text {. }
$$

Also, from (16-54) and the definition (16-45),

$$
\hat{n} \sigma_{\phi} \omega_{s}=\left(\left(n-Q_{y o}\right) \eta+\xi Q_{y o}\right) \omega_{o} \sigma_{\delta} .
$$

Now, we can use the identity $(14-24)$ to obtain

$$
\sqrt{\frac{\pi}{2}} \frac{1}{\delta \sigma_{\phi^{\omega} s}} h_{T}\left(\frac{Q}{L_{\sigma_{\phi}}}\right)=i \int_{-\infty}^{\infty} d \delta \frac{g(\delta)}{\Omega+w_{y_{0}}+\delta w_{0}\left\{\left(n-Q_{y_{0}}\right) \pi+\xi Q_{y_{0}}\right\}},
$$

hence

$$
\begin{aligned}
I_{m n}= & i \frac{c e I_{a v}}{4 \pi Q_{y o} E_{0}} z^{y}\left(n w_{0}+\Omega\right) e^{-(n-m) \sigma_{\phi}^{2} / 2} \\
& \times \int_{-\infty}^{\infty} d \delta \frac{g(\delta)}{\Omega+\omega_{0}+\delta_{0}\left\{\left(n-Q_{y o}\right) n+\xi_{y} Q_{y o}\right\}}
\end{aligned}
$$

This is the same matrix that appears in $(15-5 b)$ with $\lambda_{m-n}$ given by $(16-53)$. We have thus reduced the finite $\omega_{s}$ bunched beam transverse instability problem in the high frequency fast blowup 1 imit $(19-1)$ and (19-2) to the $\omega_{s}=0$ problem already discussed in Section 15.

\section{TRANSVERSE SYMMETRIC COUPLED BUNCH MODES}

In Section 17, we treated the single bunch head-tail mode. Here we consider how the presence of many bunches in the ring affects the conclusion of that treatment. We assume that there are $h$ identical bunches symmetrically distributed around the ring. The conclusion will be that, corresponding to each head-tail mode number $\mu$, there are h independent coherent modes, each mode being characterized by how the phases of the coherent motion of the neighboring bunches differ. 
We rely heavily on the discussion in Sections 16 and 17 ; only minor modification is needed to make it applicable to the present multibunch case. We sketch the needed modification below.

Denote by $\phi_{j}$ the location of the center of the $j$-th bunch,

$$
\phi_{j}=\frac{2 \pi}{h} j, \quad j=0,1, \ldots, h-1 .
$$

If $\Psi(j)$ is the distribution Eunction of the $j-t h$ bunch, the total distribution function is

$$
\Psi=\sum_{j=0}^{h-1}{ }_{\frac{g}{1}}(j)
$$

Since different bunches do not overlap, the vlasov equation can be written as

Define

$$
\frac{\partial \Psi^{(j)}}{\partial t}+\left(\Psi^{(j)}, H\right)=0, \quad j=0,1, \ldots, h-1 .
$$

$$
\Psi(j)=\Psi_{0}\left(J_{s}, J_{y}\right)+\Psi_{1}(j)\left(J_{s}, \Psi_{s} ; J_{y}, \alpha_{y}\right) e^{-i \Omega t} ;
$$

then the dipole density of the $j-$ th bunch is

$$
\begin{aligned}
D^{(j)}(\phi, t)= & \int d J_{s} d \psi_{s} d J_{y} d \psi_{y} \delta\left(\phi-\phi_{j}-\bar{\phi} \cos \psi_{s}\right) \\
& x y \Psi_{1}(j)\left(j_{s}, \psi_{s} ; J_{y}, \alpha_{y}\right) e^{-i \Omega t},
\end{aligned}
$$

and its Fourier component is

$$
\eta_{n}^{(j)}(\Omega)=\bar{D}_{\mathfrak{n}}^{(j)}(\Omega) e^{-i n \phi} j
$$

with

$$
\bar{D}_{n}^{(j)}(\Omega)=\frac{1}{2 \pi} \int d J_{s} d \psi_{s} d J_{y} d \psi_{y} y^{\psi}{ }_{1}^{(j)} e^{-i n \bar{\phi} \cos \psi_{s}} .
$$

Note that, from the assumption of identical bunches, we take $\psi_{0}$ in Eq. (20-4) to be independent of $j$.

Let us adopt the normalization

$$
\int d J_{s} d \psi_{s} d J_{y} d \psi_{y} \Psi^{(i)}\left(J_{s}, \psi_{s} ; J_{y}, \alpha_{y}\right)=1 \text {. }
$$

Then the force field induced by the collective motion of the bunches is

$$
F_{y}(\theta, t)=i \frac{\omega_{0}}{c} I_{B} \sum_{k=0}^{h-1} \sum_{n} D_{n}^{(k)}(\Omega) z^{y}\left(n \omega_{0}+\Omega_{1}\right) e^{i n \phi-i \Omega t},
$$


where $I_{B}=I_{a y} / h$ is the average current per bunch. For the force on the particle in the j-th bunch, $\mathrm{Eq} \cdot(20-8)$ should be evaluated at

$$
\theta=\omega_{0} t+\phi_{j}+\bar{\phi} \cos \psi_{s} \quad \text { or } \quad \phi=\phi_{j}+\bar{\phi} \cos \psi_{s} \text {. }
$$

Define

$$
\Psi_{1}^{(j)}=e^{-i \alpha_{y} \Phi^{(j)}}\left(J_{s}, J_{y}, \Psi_{s}\right)
$$

then the linearized Vlasov equation becomes

$$
\begin{aligned}
& -i\left(\Omega+\omega_{y o}\right) \Phi^{(j)}-\omega_{s} \frac{\partial \Phi^{(j)}}{\partial \psi_{s}}=\frac{i}{2} e^{i a Q_{y o} \bar{\phi} \cos \psi_{s \bar{y}}} \frac{\partial \Psi_{0}}{\partial J_{y}}{ }_{y}(j) \\
& F_{y}^{(j)}\left(J_{s}, \|_{s}\right)=i \frac{e \omega_{o}}{c} I_{B} \sum_{k} \sum_{n} \vec{D}_{n}^{(k)} \\
& \times z^{y}\left(n \omega_{o}+\Omega\right) e^{i n\left(\phi_{j}-\phi_{k}\right)} e^{i n \bar{\phi} \cos \psi_{s}} \text {. }
\end{aligned}
$$

For a Gaussian bunch, we arrive at the following secular equation for the $\mu$-th head-tail mode:

where

$$
\bar{D}_{\mathbf{m}}^{(j)}=\frac{1}{h} \sum_{\mathbf{n}} \sum_{k} T_{\min }^{(j, k)} \bar{D}_{n}^{(k)}
$$

$$
T_{\operatorname{mn}}^{(j, k)}=e^{i n\left(\phi_{j}-\phi_{k}\right)} T_{\min }=e^{i n \frac{2 \pi}{h}(j-k)} T_{\min } \text {, }
$$

with

$$
\begin{aligned}
T_{\operatorname{mn}}= & i \frac{c}{4 \pi Q_{y o} \omega_{s} E_{0}} e I_{a v} z^{y}\left(n \omega_{0}-\omega_{y o}-\mu_{s}\right) \\
& \times \frac{1}{Q-\mu} e^{-\left(\sim^{2}+\Omega^{2}\right) \sigma_{\phi}^{2} / 2} I_{\mu}\left(\tilde{n} \sigma_{\phi}^{2}\right) .
\end{aligned}
$$

Equation (20-12) with (20-13) can easily be diagonalized in the $h$ dimensional $(j)$-space. The eigenvector in $(j)$-space is

$$
\bar{D}_{m}^{(j)}=\bar{D}_{m}^{(0)} e^{i \frac{2 \pi}{h} s j},
$$

where the parameter $s, s=0,1, \ldots, h-1$, labels different eigensolutions. 
75

From the identity

$$
\because \sum_{k=0}^{h-1} e^{i \frac{2 \pi}{h}(n-S)(j-k)}=h \sum_{\ell=-\infty}^{\infty} \delta n-s, \ell h
$$

Eq. (20-12) and (20-13) reduce to

$$
\bar{D}_{\mathrm{mh}+\mathrm{S}}^{(0)}=\sum_{\mathrm{n}} \mathrm{T}_{\mathrm{mh}+\mathrm{S}, \mathrm{nh}+\mathrm{S}} \overline{\mathrm{D}}_{\mathrm{nh}+\mathrm{S}}^{(0)}
$$

$(20-17)$

Note that Eq. $(20-14)$ is identical to $(17-4)$. Also, $(20-17)$ is the same as (16-43) except for the modification of the subscripts. Thus, we obtain for the S-th coupled bunch mode the following expression for the coherent frequency shift in the small bunch approximation:

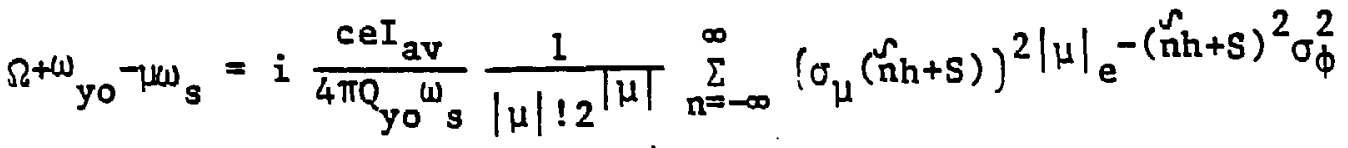

$$
\begin{aligned}
& \times \mathrm{z}^{\mathrm{y}}\left(\left(\mathrm{nh}+\mathrm{s}-Q_{\mathrm{yo}_{0}}\right) w_{0}+w_{s}\right) .
\end{aligned}
$$

$(20-18)$ 
76

APPENDIX A MATHMETICAL FORMULAE

If

$$
i Q \psi(\theta)+\frac{\partial \psi(\theta)}{\partial \theta}=F(\theta) \text { with } F(\theta+2 \pi)=E(\theta),
$$

then the periodic solution of this equation is

$$
\begin{aligned}
& \psi(\theta)=-\frac{1}{1-e^{i 2 \pi Q}} \int_{0}^{2 \pi} d \theta^{\prime} e^{i Q \theta^{\prime}} F\left(\theta+\theta^{\prime}\right) . \\
& \int_{0}^{2 \pi} d \theta^{\prime} e^{i x \cos \left(\theta+\theta^{\prime}\right)-i y \cos \theta^{\prime}}=2 \pi J\left(\sqrt{x^{2}+y^{2}-2 x y \cos \theta}\right) \text {. } \\
& (A-2) \\
& \int_{0}^{2 \pi} d \theta^{\prime} \sin \left(\theta+\theta^{\prime}\right) \mathrm{e}^{i x \cos \left(\theta+\theta^{\prime}\right)-i y \cos \theta !} \\
& =-i 2 \pi \frac{y \sin \theta}{\sqrt{x^{2}+y^{2}-2 x y \cos \theta}} J_{1}\left(\sqrt{x^{2}+y^{2}-2 x y \cos \theta}\right) . \\
& \int_{0}^{\infty} r d r e^{-r^{2} / 2 \sigma^{2}} J_{0}(\alpha r)=\sigma^{2} e^{-\alpha^{2} \sigma^{2} / 2} \\
& \int_{0}^{\infty} r^{2} d r e^{-r^{2} / 2 \sigma^{2}} J_{1}(\alpha r)=\infty e^{4} e^{-\alpha^{2} \sigma^{2} / 2} \\
& e^{i Q \theta}=\frac{i}{2 \pi}\left(1-e^{i 2 \pi Q}\right) \sum_{\mu=-\infty}^{\infty} \frac{1}{Q-\mu} e^{i \mu \theta}, \quad(0<\theta<2 \pi) . \quad(A-6) \\
& \int_{0}^{2 \pi} d \psi e^{x \cos \psi} e^{-i \mu \psi}=2 \pi I_{\mu}(x) \text {. } \\
& (A-7) \\
& I_{-\mu}(x)=I_{\mu}(x) \\
& \int_{0}^{2 \pi} d \psi e^{i Q \psi} e^{x \cos \psi}=i\left(1-e^{i 2 \pi Q}\right) \sum_{\mu=-\infty}^{\infty} \frac{1}{Q-\mu} I_{\mu}(x) . \\
& \int_{0}^{2 \pi} d \psi_{\sin } \psi e^{i Q \psi} e^{x \cos \psi}=-\frac{1}{x}\left(1-e^{i 2 \pi Q}\right) \sum_{\mu=-\infty}^{\infty} \frac{\mu}{Q-\mu} I_{\mu}(x) \cdot(A-10)
\end{aligned}
$$




\section{APPENDIX B WEAR FOCUSING SYNCAROTRON}

This appendix deals with the Hamiltonian Eormalism of the motion of a particle in a weak focusing machine, including the effect of the sextupole magnet and the if accelerating cavity. Specifically, it shows how the well-known Hamiltonian for the Lorentz force transforms into the form used in Section 16.

\section{B.1 Expansion of Hamiltonian}

The Hamiltonian of the particle trajectory in the Serret-Frenet coordinate system is ${ }^{6,7}$

$$
H=c \sqrt{\frac{\left(p_{s}-e A_{s}\right)^{2}}{(1+k x)^{2}}+p_{x}^{2}+p_{y}^{2}+m^{2} c^{2}}
$$

where $K=1 / R$ is the constant curvature of the reference circular orbit, $s$ measures the length along the same orbit, and ( $\left.p_{x}, p_{y}, p_{s}\right)$ are the canonical momenta conjugate to $(x, y, s)$. We choose the convention that $\hat{x} \times \hat{y}=\hat{s}$, where $\hat{x}, \hat{y}$, and $\hat{s}$ are unit vectors. We are using time as the independent variable.

The vector potential $A_{s}$ can be split into three parts:

$$
A_{s}(x, y, t)=A_{L}(x, y)+A_{\text {sext }}(x, y)+A_{I f}(s, t) \text {. }
$$

Following Ref. 7, we define the linear part of the accelerator by

$$
e A_{L}=-p_{0}\left\{k x+\frac{1}{2} k^{2}\left(n x^{2}+n y^{2}\right)-\frac{n}{3} k^{3} x^{3}+\frac{n}{2} k^{3} x y^{2}\right\},
$$

where $P_{0}=\sqrt{E_{0}^{2}-m^{2} e^{4}} / c=B_{o} E_{o} / c$ is the nominal momentum, and the $n^{\prime} s$ are related to-the focusing magnetic field $B$ by

$$
\begin{aligned}
& n=-\frac{1}{K}\left(\frac{\partial}{\partial x} \ln B_{y}\right)_{x=y=0}, \\
& a_{x}=1-a .
\end{aligned}
$$

The rf voltages can be described in the smooth approximation* by

$$
\mathrm{eA}_{\mathrm{If}}=\frac{\mathrm{p}_{o}}{2 n \beta_{o}^{2} c^{2}} \omega_{s}^{2}\left(s-\beta_{o} c t\right)^{2}
$$

*Since the rf cavity is localized, Arf can be represented as a superposition of propagating waves around the ring with angular phase velocity $h \omega_{0} / n, \pi=0, \pm 1, \pm 2, \ldots$. The smooth approximation consists of keeping only the wave with its phase velocity equal to the particle velocity; namely, the wave with $\mathrm{n}=\mathrm{h}$. 
with the angular synchrotron frequency $\omega_{s}$ given by

$$
\omega_{s}^{2}=-\frac{e_{\pi} \omega_{O}^{2} h}{2 \pi \beta_{0}^{2} E} \hat{v}_{\mathrm{rf}} \cos \phi_{s}
$$

where $h$ is the harmonic number, $\hat{v}_{r f}$ is the peak rf voltage, and $\phi_{S}$ is its phase. The sextupole field is given by

$$
\text { eA } A_{\text {sext }}=\frac{b_{3}}{3} P_{0} K^{3}\left(x^{3}-3 x y^{2}\right) \text {, }
$$

with $b_{3}$ describing the sextupole strength.

It is convenient to introduce the momentum deviation

$$
\overline{\mathrm{p}}=\mathrm{p}_{\mathrm{s}}-\mathrm{p}_{\mathrm{O}} \quad \text { and } \delta=\overline{\mathrm{p}} / \mathrm{p}_{\mathrm{O}} \text {. }
$$

Let us now take $p_{0} \gg m c$ and expand_Eq. $(B-1)$ in the rest of the variables. To third order in $x, y$, and $\bar{p}$, and first order in $A$, we obtain

$$
\begin{aligned}
B= & E_{s}+\frac{P_{s} P_{o} c^{2}}{2 E_{s}}\left\{\left(n_{x}+2 \delta\right) k^{2} x^{2}+n k^{2} y^{2}\right\}-\frac{P_{s} \bar{p} c^{2}}{E_{s}} k x \\
& +\frac{c^{2}}{2 E_{s}}\left(p_{x}^{2}+p_{y}^{2}\right)+\frac{P_{s} P_{o} c^{2}}{3 E_{s}}\left\{\frac{n-3}{2} k^{3} x^{3}-b_{3} k^{3}\left(x^{3}-3 x y\right)\right\} \\
& -\frac{P_{s} c^{2}}{E_{s}} e A_{E f},
\end{aligned}
$$

with

$$
E_{s}=\sqrt{P_{s}^{2} c^{2}+m^{2} c^{4}}
$$

From

$$
\begin{aligned}
& E_{s} \simeq E_{0}+B_{0} c p_{0}\left(\delta+\frac{\delta^{2}}{2 \gamma_{0}^{2}}\right), \\
& \frac{1}{E_{s}} \simeq \frac{1}{E_{0}}\left\{1-\left(1-\frac{1}{\gamma_{0}^{2}}\right) \delta\right\},
\end{aligned}
$$

and

$$
\frac{P_{s}}{E_{s}}=\frac{B_{0}}{c}\left(1+\frac{\delta}{\gamma_{0}^{2}}\right),
$$


with $\gamma_{0}=E_{0} / \mathrm{mc}^{2}, E q .(B-10)$ becomes

$$
\begin{aligned}
H= & \left.E_{0}+\beta_{0} c p_{0}\left(\left(\delta+\frac{\delta^{2}}{2 \gamma_{0}^{2}}\right)+\left(1+\frac{\delta}{\gamma_{0}^{2}}\right) \frac{1}{2}\left[n_{x}+2 \delta\right) k^{2} x^{2}+n k^{2} y^{2}\right]-\delta k x\right) \\
& +\frac{c^{2}}{2 E_{0}}\left\{1-\left(1-\frac{1}{\gamma_{0}^{2}}\right) \delta\right\}\left(p_{x}^{2}+p_{y}^{2}\right) \\
& +\frac{1}{3} \beta_{o} c p_{0}\left\{\frac{n-3}{2} k^{3} x^{3}-b_{3} k^{3}\left(x^{3}-3 x y^{2}\right)\right\}-\beta_{o} c e A f f
\end{aligned}
$$

where we have ignored the terms of orders $\delta \kappa_{x} / \gamma_{0}, \delta_{A_{I f}} / \gamma_{0}^{2}$, and $\delta k^{3} x^{3} / \gamma_{0}{ }^{2}$.

\section{B. 2 Closed Orbit}

The quantity $x$ consists of two parts:

$$
x=x_{c}+x_{\beta}
$$

where $x_{c}$ is the closed orbit, and $x_{\beta}$ describes the horizontal betatron oscillation around $x_{c}$. . $x_{c}$ is determined by the requirement that.it be proportional to $\delta$ (we consider only the linear closed orbit) and that upon substitution of $(B-13)$ into (B-12), terms proportional to $x_{\beta} \delta$ in the resulting Hamiltonian cancel out. From inspection, we see

$$
\mathrm{x}_{\mathrm{c}}=\frac{1}{\mathrm{kn}} \delta \text {. }
$$

The above decomposition of $x$ can be achieved by the canonical transformation $\left(x, p_{x}, s, p_{s}\right) \rightarrow\left(x_{\beta}, p_{x_{\beta}}, s_{1}, p_{s_{1}}\right)$ generated by 41

$$
F_{3}=-\left(x_{B}+\frac{\delta}{n_{x} k}\right) p_{x}-s_{1} p_{s}
$$

or

$$
\begin{aligned}
& p_{x_{B}}=-\frac{\partial F_{3}}{\partial x_{B}}=p_{x}, \quad p_{s_{1}}=-\frac{\partial F_{3}}{\partial s_{1}}=p_{s}, \\
& x=-\frac{\partial F_{3}}{\partial p_{x}}=x_{B}+\frac{\delta}{n_{x} k}, \\
& s=-\frac{\partial F_{3}}{\partial p_{s}}=s_{1}+\frac{1}{n_{x} k} \frac{p_{x}}{p_{0}} .
\end{aligned}
$$


We see that Eq. (B-16b) gives the desired decomposition and that, from $(B-16 a), P_{x}$ and $P_{s}$ are not changed under this transformation. Equation $(B-16 C)$ introduces, on the other hand, a coupling between the horizontal and longitudinal directions. Note that the Hamiltonian $B$ depenas on $s$ only through $A_{r f}$; this $x-s$ coupling may indeed excite the synchro-betatron resonance through the $r f$ cavity. We assume, however, that we are far away from these resonances and ignore the last term in $(B-16 c)$.

We thus obtain an expression for $H$ which is explicit in $x_{\beta}$ :

$$
\begin{aligned}
H= & E_{0}+\beta_{0} c p_{0} \frac{1}{2} \kappa^{2}\left(x_{\beta}^{2}\left\{n_{x}-\delta\left(\frac{n+1+2 b_{3}}{n_{x}}-\frac{n_{x}}{\gamma_{0}^{2}}\right)\right\}\right. \\
i & \left.+y^{2}\left(n+\delta\left(\frac{2 b_{3}}{n_{x}}+\frac{n}{\gamma_{0}^{2}}\right)\right\}\right)+\frac{1}{2} \beta_{0} c\left\{1-\left(1-\frac{1}{\gamma_{0}^{2}}\right) \delta\right\} \\
& \frac{\left(p_{x}^{2}+p_{y}^{2}\right)}{p_{0}}+\beta_{0} c p_{0}\left(\delta-\frac{1}{2}\left(\frac{1}{n_{x}}-\frac{1}{\gamma_{0}^{2}}\right) \delta^{2}\right)-\beta_{0} c e A_{r f},
\end{aligned}
$$

where the terms of orders $\delta^{3}, \delta^{2} x_{B}, x_{B}^{3}, x_{B} y^{2}, \delta x_{B}^{2} / Y_{0}^{2}$, and $\delta y^{2} / Y_{0}^{2}$ are ignored.

Let us now find the nominal tune $Q_{0}$, the momentum compaction factor $\alpha$, and the chromaticity $\xi$ corresponding to Eq. (B-17). From Hamilton's equation,

$$
\dot{s}=\frac{\partial H}{\partial P_{s}}=\frac{1}{F_{0}} \frac{\partial H}{\partial \delta}=\beta_{o} c\left(1-\left(\frac{1}{n_{x}}-\frac{1}{\gamma_{0}^{2}}\right) \delta\right) .
$$

or, in terms of $\theta=k s=s / R$,

$$
\delta=\omega_{0}\left(1-\left(\frac{1}{n_{x}}-\frac{1}{\gamma_{0}^{2}}\right) \dot{0}\right) .
$$

Therefore,

$$
Y_{t}=\frac{1}{\sqrt{n_{x}}}, \quad \alpha=\frac{1}{n_{x}}, \quad \eta=\frac{i}{n_{x}}-\frac{1}{\gamma_{0}^{2}} .
$$

Hamilton's equation also gives the following equarions of motion valid to first order in $\delta$ : 


$$
\begin{aligned}
& \ddot{x}_{\beta}+w_{0}^{2}(1-n \delta)^{2} n_{x}\left(1-\frac{n+n^{2}+2 b_{3}}{n_{x}^{2}}\right) x_{\beta}=0, \\
& \ddot{y}+w_{0}^{2}(1-n \delta)^{2} n\left\{1+\left(\frac{n+1}{n_{x}}+\frac{2 b}{n n_{x}}\right) \delta\right\} y=0,
\end{aligned}
$$

Therefice, the nominal tunes are

$$
Q_{x o}=\sqrt{n_{x}}, \quad Q_{\text {yo }}=\sqrt{n},
$$

and the linear chromaticities are

$$
\xi_{x}=-\frac{n+n^{2}}{2 n_{x}^{2}}-\frac{b_{3}}{n_{x}^{2}} \quad \text { and } \quad \xi_{y}=\frac{n+1}{2 n_{x}}+\frac{b_{3}}{n n_{x}} \text {. }
$$

The terms in Eq. (B-22) that are proportional to $b_{3}$ are the chromaticities induced by the sextupole magnet, and the remainders are called the natural chromaticities.

The Hamiltonian $(B-17)$ can now be written as

$$
\begin{aligned}
H= & E_{0}+\beta_{0} c P_{0} \frac{1}{2}\left(\left(n_{x} k^{2} x_{B}^{2}+\frac{p_{x}^{2}}{P_{0}^{2}}\right)\left\{1+\left(\xi_{x}-\eta\right) \delta\right\}+(x+y)\right) \\
& +\beta_{0} C P_{0}\left(\delta-\frac{1}{2}\left\{\pi \delta^{2}+\frac{\omega_{s}^{2}}{\pi B_{0}^{2} c^{2}}\left(s-\beta_{0} c t\right)^{2}\right\}\right)+H^{(1)},
\end{aligned}
$$

with

$$
\begin{aligned}
E^{(1)}= & \beta_{0} c p_{0} \frac{\delta}{2}\left[\left(n_{x} k^{2} x_{B}^{2}-\frac{p_{x}^{2}}{p_{0}^{3}}\right)\left(\frac{n^{2}-3 n}{2 n_{x}}-\frac{b_{3}}{n_{x}^{2}}\right)\right. \\
& \left.+\left(n \kappa^{2} y^{2}-\frac{p_{y}^{2}}{p_{0}^{2}}\right)\left(\frac{1}{2}+\frac{b_{3}}{n_{x}}\right)\right) .
\end{aligned}
$$

$$
\begin{aligned}
& \text { Exercise: Show that } \mathrm{H}^{(1)} \text { does not contribute to }(B-20) \\
& \text { to first order in } \delta \text {. }
\end{aligned}
$$

We shall ignore $\mathrm{H}^{(1)}$. 
B.3 A Transformation of the Synchrotron Variable

It is convenient to use the variable $\phi$ instead of $s$ to describe the synchrotron motion. Let us carry out the canonical transformation $\left(s, P_{s}, H\right)+(\phi, H, \bar{H})$ generated by

$$
F_{2}=\left(s-B_{0} c t\right)\left(K W+p_{0}\right)
$$

We have

$$
\begin{aligned}
& P_{s}=\frac{\partial F_{2}}{\partial s}=K W+P_{0}, \\
& \phi=\frac{\partial F_{2}}{\partial W}=k\left(s-B_{0} c t\right) .
\end{aligned}
$$

The new Hamiltonian is

$$
\begin{aligned}
& \bar{H}=\mathrm{H}+\frac{\partial F_{2}}{\partial t}=\frac{1}{2}\left(\left\{\left(\frac{c^{2}}{E_{0}}\right) p_{x}^{2}+Q_{x o}^{2} \omega_{\circ}^{2}\left(\frac{E_{0}}{c^{2}}\right) x_{B}{ }^{2}\right\}\right. \\
& \left.x\left\{1+\left(\xi_{x}-\eta\right) \frac{\omega_{0}}{\beta_{0}^{2} E} w\right\}+(x+y)\right)-\frac{1}{2}\left(\bar{\eta} \omega^{2}+\omega_{s}^{2} \phi^{2} / \bar{\eta}\right),
\end{aligned}
$$

with

$$
\bar{\eta}=\pi \omega_{0}^{2} /\left(B_{0}^{2} E_{0}\right) \text {. }
$$

It is useful to write Eq. (B-26a) in another form. If the last term in $(B-11 a)$ is ignored, the resulting equation together with $(B-26 a)$ gives

$$
\mathrm{W}=\Delta \mathrm{E} / \mathrm{w}_{0} \text {, }
$$

where $\Delta E=E_{S}-E_{0}$. 


\section{APPENDIX C PANOFSRY-WENTZEL THEOREM}

Panofsky and Wentzel 38 established a relationship between the transverse kick a stiff particle (a fast particle) receives and the energy it loses when it passes through a cavity. We rewrite their theorem in this appendix in a form more suitable for our discussion; we relate the transverse component of the Lorentz force to the electric fields around the ring.

It is convenient to work with the Hamiltonian formalism where $s$ instead of $t$ is the independent variable. In Serret-Frenel coordinate system, the Hamiltonian is 6,7

$$
\mathscr{H}=-\mathrm{eA}_{s}-(1-k x) \sqrt{\frac{(E-e V)^{2}}{c^{2}}-\mathrm{m}^{2} c^{2}-\left(\vec{P}_{\mathrm{T}}-\mathrm{eA}{ }_{\mathrm{T}}\right)^{2}}
$$

where $\overrightarrow{\mathscr{P}}_{\mathrm{T}}$ and $\vec{A}_{\mathrm{T}}$ are two-dimensional transverse vectors $\left(\mathscr{P}_{\mathrm{x}}, \mathscr{P}_{\mathrm{y}}\right.$ ) and $\left(A_{x}, A_{y}\right)$, respectively, and $k$ is the curvature of the reference orbit.

We ignore the effects of the curvature and work in the gauge where the scalar potential $\mathrm{V}=0$. Thus $\mathrm{Eq}$. (C-1) becomes

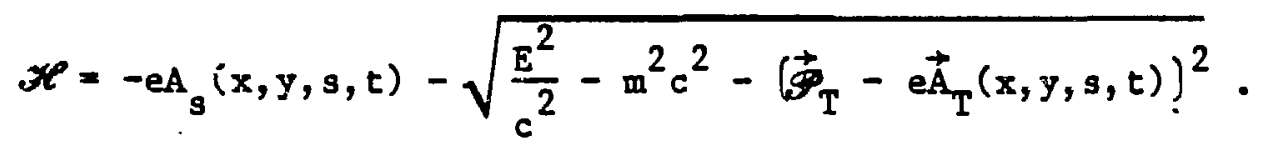

The canonical variables of this Hamiltonian system are $\left(x, \mathscr{P}_{\mathbf{x}}\right)$, $\left(y, \mathscr{P}_{\mathrm{y}}\right)$ and $(t,-E)$. The transverse kinetic momentum $\overrightarrow{\mathrm{P}}_{\mathrm{T}}$ is related to the canonical momentum $\mathcal{F}_{\mathrm{T}}$ by

$$
\overrightarrow{\mathrm{p}}_{\mathrm{T}}=\vec{S}_{\mathrm{T}}-\overrightarrow{e A}_{\mathrm{T}}(\mathrm{x}, \mathrm{y}, \mathrm{s}, \mathrm{t}) \text {. }
$$

From Hamilton's equation

$$
\frac{d \Phi_{y}}{d s}=-\frac{\partial \mathscr{H}}{\partial y}=e \frac{\partial A}{\partial y}+\frac{e}{p_{s}} \vec{p}_{T} \cdot \frac{\partial}{\partial y} \vec{A}_{T},
$$

where $p_{s}=\sqrt{E^{2} / c^{2}-m^{2} c^{2}-P_{T}{ }^{2}}$. We ignore the last term of $E q$. $(c-4)$ on the ground that $\mathrm{p}_{\mathrm{T}} / \mathrm{p}_{\mathrm{S}}$ is small. We thus have

$$
\frac{d S_{y}}{d s} \simeq \mathrm{e} \frac{\partial}{\partial y} A_{s} \text {. }
$$

Similarly,

$$
\frac{d E}{d s}=\frac{\partial \mathscr{H}}{\partial t}=-\mathrm{e} \frac{\partial A}{\partial t} \text {. }
$$

From Eqs. (C-5) and (C-6),

$$
\frac{\partial}{\partial t} \frac{\mathrm{d} \mathscr{P}_{\mathrm{y}}}{\mathrm{ds}}=-\frac{\partial}{\partial \mathrm{y}} \frac{\mathrm{dE}}{\mathrm{ds}} \text {. }
$$


84

Let us write this relation in a more transparent form. $d / d s$ in Eq. $(C-7)$ is the total derivative following the particle. Therefore, $\mathrm{dE} / \mathrm{ds}$ is the energy gain of the particle per unit length. $0 \mathrm{r}$,

$$
\frac{d E}{d s}=e \mathscr{E}_{s}(x, y, s, t)
$$

with $\mathscr{E}_{s}(x, y, s, t)$ the longitudinal electric field. Similarly,

$$
\frac{d p_{y}}{d s}=\frac{1}{\beta_{0} c} \frac{d p_{y}}{d t}=\frac{1}{\beta_{0} c} F_{y}(x, y, s, t),
$$

where $F_{y}$ is the transverse Lorentz force field in the $y$-direction, and $B_{o} c$ is the particle speed. In the present gauge, the $y$-component of the electric field is

$$
\mathbb{E}_{y}(x, y, s, t)=-\frac{\partial}{\partial t} A_{y}(x, y, s, t) \text {. }
$$

Substituting the $y$-component of $(c-3)$ into $(c-7)$, and then applying $(\mathrm{C}-8),(\mathrm{C}-9)$ and $(\mathrm{C}-10)$ in the resulting equation, we obtain

$$
\frac{\partial}{\partial t} F_{y}(x, y, s, t)=e \frac{d}{d t} \mathscr{E}_{y}(x, y, s, t)-e \beta_{o} c \frac{\partial}{\partial_{y}} \mathscr{E}_{s}(x, y, s, t) \cdot .(c-11)
$$

This is the Panofsky-Wentzel theorem. Note that

$$
\frac{d}{d t} \mathscr{E}_{y}(x, y, s, t)=\beta_{o} c \frac{\partial}{\partial s} \mathscr{E}_{y}(x, y, s, t)+\frac{\partial}{\partial t} \mathscr{E}_{y}(x, y, s, t) . \quad(c-12)
$$




\section{APPENDIX D NASSIBIAN-SACHERER RELATION}

In this appendix, we apply the Panofsky-Wentzel theorem (C-12) to prove the Nassibian-Sacherer relation, 39 which relates the transverse impedance discussed in Section 13 to a generalized longitudinal

impedance. Later we use the Nassibian-Sacherer relation to prove that the resistive part (real part) of the transverse inpedance $z_{n}(\omega)$ is positive for $n>0$ and negative for $n<0$. $I(\theta, t)$ by

Let $\mathrm{J}_{\mathrm{g}}(\mathrm{x}, \mathrm{y}, \theta, t)$ be the current density related to the current

$$
I(\theta, t)=\int \operatorname{dxdy} J_{s}(x, y, \theta, t) .
$$

Consider a filament of the beam current at the transverse position $\left(x_{0}, y_{0}\right)$ given by

$J_{s}(x, y, \theta, t)=\delta\left(x-x_{0}\right) \delta\left(y-y_{0}\right) \sum_{n=-\infty}^{\infty} \int d \omega I_{n}(\omega) e^{i n \theta-i \omega t}$.

Denote by $\mathscr{E}_{S}(x, y, \theta, t)$ the longitudinal electric field produced by the current $(D-2)$, and define a generalized longitudinal impedance $z_{n}^{L}\left(x, y ; x_{0} ; y_{0} ; w\right)$ by

$\delta_{s}(x, y, \theta, t)=-\frac{1}{2 \pi R} \sum_{n=-\infty}^{\infty} \int_{\infty}^{\infty} d w z_{n}^{L}\left(x, y ; x_{0}, y_{0} ; \omega\right) I_{n}(\omega) e^{i n \theta-\omega t}$.

One can use Lorentz's reciprocity theorem 45 to prove that the generalized impedance is symetric:

$$
z_{n}^{L}\left(x, y ; x_{0}, y_{0} ; \omega\right)=z_{n}^{L}\left(x_{0}, y_{0} ; x, y ; \omega\right)
$$

\section{Exercise: Prove $(D-4)$.}

The longitudinal impedance $z(\omega)$ defined in Section 3 is obviously related to the generalized impedance by

$$
z_{n}(w)=z_{n}^{L}\left(x_{0}, y_{0} ; x_{0}, y_{0} ; w\right) \text {. }
$$

For a general current density $J_{s}(x, y, \theta, t)$ which is distributed in the $(x, y)$ space, we have, from the superposition principle 
$\mathscr{E}_{s}(x, y, \theta, t)=-\frac{1}{2 \pi R} \sum_{n} \int d x^{\prime} d y d \omega z_{n}^{L}\left(x, y ; x^{\prime}, y^{\prime} ; \omega\right) J_{n}\left(x^{\prime}, y^{\prime}, \omega\right) e^{i \theta-i n \omega t}$,

where

$$
J_{s}(x, y, \theta, t)=\sum_{n} \int_{d w J_{n}}(x, y, w) e^{i n \theta-i w t} .
$$

Now consider the particle density

$$
\begin{aligned}
& \rho(x, y, \phi, t)=\frac{1}{2 \pi} \delta\left(x-x_{0}\right) \delta\left(y-y_{0}-D e^{i n \phi-i \Omega t}\right), \\
& =\frac{1}{2 \pi} \delta\left(x-x_{0}\right) \delta\left(y-y_{0}\right)-\frac{D}{2 \pi} e^{i n \phi-i \Omega t} \delta\left(x-x_{0}\right) \delta^{\prime}\left(y-y_{0}\right),
\end{aligned}
$$

which is normalized to 1 :

$$
\int d x d y \int_{0}^{2 \pi} d \phi \rho(x, y, \phi, t)=1 \text {. }
$$

As always in these notes, $\phi$ is related to $\theta$ by $\theta=\omega_{0} t+\phi$. Equation $(D-8)$ describes a beam filament located at $\left(x_{0}, y_{0}\right)$ and oscillating in the $y$-direction with displacement $\operatorname{Dexp}(i n \phi-i \Omega t)$. Suppose the beam consists of $\mathrm{N}$ particles; then the longitudinal current density and the dipole density corresponding to $(D-8)$ are, respectively,

$J_{s}(x, y, \theta, t)=-\frac{e N D}{2 \pi}\left(\omega_{0}+\frac{\Omega}{n}\right) \delta\left(x-x_{0}\right) \delta^{\prime}\left(y-y_{0}\right) e^{i n \theta-i \omega t}$,

and

$$
D(\phi, t)=\frac{1}{2 \pi} D e^{i \pi \phi-i \Omega t},
$$

with $\omega=n \omega_{0}+\Omega$.

The longitudinal electric field generated by Eq. (D-10) can be calculated by using $(D-6)$. It is

$$
\tilde{E}_{s}(x, y, \theta, t)=-\frac{e N D}{4 \pi^{2} R n} \frac{\omega}{n} \frac{\partial}{\partial y_{0}} z_{n}^{L}\left(x, y ; x_{0}, y_{0} ; \omega\right) e^{i n \theta-i \omega t} .
$$

We now use the Panofsky-Wentzel theorem to find the transverse force field $F_{y}(x, y, \theta, t)$. Following Nassibian and Sacherer, we ignore the electric deflection; that is, we set $\mathscr{E}_{y}=0$ in Eq. (c-11). This 
means that the following discussion does not apply, for example, to the space charge impedance. Equation (c-11) now becomes

$\frac{\partial}{\partial t} F_{y}(x, y, \theta, t)=\frac{e^{2} \beta_{o} c N D}{4 \pi^{2} R} \frac{\omega}{n} \frac{\partial^{2}}{\partial y \partial y_{0}} z_{n}^{L}\left(x, y ; x_{0}, y_{0} ;(\omega) e^{i n \theta-i \omega t}\right.$.

$F_{y}$ is clearly proportional to $\exp (i n \theta-i \omega t)$. Thus the solution of Eq. $(D-13)$ is

$$
F_{y}(x, y, \theta, t)=i \frac{e D}{2 \pi n} I_{a v} \frac{\partial^{2}}{\partial y \partial y_{0}} z_{n}^{L}\left(x, y ; x_{o}, y_{o} ; \omega\right) e^{i n \theta-i \omega t} \cdot \quad(D-14)
$$

Now we are ready to find the relationship between the transverse impedance $\mathrm{z}_{\mathrm{y}}^{\mathrm{y}}(\omega)$ and the generalized longitudinal impedance. From Eqs. $(13-8)^{n}$ and $(D-11)$,

$F_{y}\left(x_{0}, y_{0}, \theta, t\right)=i \frac{e \omega}{c} I_{a v} z_{n}^{y}\left(n \omega_{0}+\Omega\right) \cdot \frac{D}{2 \pi} e^{i n \phi-i \Omega t}$.

Comparing Eqs. (D-14) and (D-15), we obtain

$$
z_{n}^{y}(\omega)=\frac{c}{n \omega}\left(\frac{\partial^{2}}{\partial y \partial y_{0}} z_{n}^{L}\left(x, y ; x_{0}, y_{0} ; \omega\right)\right)_{y=y_{0}, x=x_{0}} .
$$

This is the Nassibian-Sacherè relation.

When a beam passes through a passive device, it can lose but cannot gain energy. We saw in section 3 that this condition implies that the resistive part of the longitudinal impedance must be positive for all $n$ and $w$. In the following, we investigate what this condition implies for the resistive part of the transverse impedance.

From the above condition,

$$
\operatorname{Real}\left(\int \mathrm{d} x \mathrm{~d} y \mathscr{E}_{s}(\mathrm{x}, \mathrm{y}, \theta, t) \mathrm{J}_{s}^{*}(\mathrm{x}, \mathrm{y}, \theta, t)\right) \leq 0 .
$$

Substituting $(D-10)$ and (D-12) into this inequality, we obtain

$$
\operatorname{Real}\left(\left.\frac{\partial^{2}}{\partial y^{\partial} y_{0}} z_{n}^{L}\left(x, y ; x_{0}, y_{0} ; w\right)\right|_{\substack{y=y_{0} \\ x=x_{0}}}\right) \geq 0 \text {. }
$$

From $(D-18)$ and $(D-16)$, we obtain

$$
\begin{aligned}
\mathscr{R}_{n}^{\mathrm{y}}(\omega) & \geq 0, & & \text { if } \mathrm{n}>0 \\
& \leq 0, & & \text { if } \mathrm{n}<0
\end{aligned}
$$

where $\mathscr{R}_{\mathrm{n}}^{\mathrm{y}}$ is the real part of $\mathrm{z}_{\mathrm{n}}^{\mathrm{y}}$ 


\section{PRINCIPAL SYMBOLS}

a

$a_{s}, \frac{\alpha}{7}$

B

$\beta_{0}$

c

$D(\phi, t)$

$D_{\mathrm{n}}(\Omega)$

$\delta$

$\mathbf{E}$

$E_{0}$

$\mathscr{E}$ or $\mathscr{E}_{s}$

$6_{y}$

$\varepsilon$

ก

$\bar{n}$

$\phi$

$\bar{\phi}$

$F_{y}$

$\mathcal{F}, \mathscr{F}_{\mathrm{BL}}, \mathscr{F}_{\mathrm{s}}$

$g(\delta)$

$\gamma$

$\gamma_{\varepsilon}$

h
A dimensionless parameter that sets the scale of the betatron phase modulation due to synchrotron oscillation, $a=\xi / \eta-1(16-11)$.

Rolmogorov transformed angle-variables $(16-19,20)$.

Velocity of a particle in units of $c$.

Nominal value of $\beta$.

Speed of light.

Dipole density (13-2).

Fourier component of $D(\phi, t)(13-7)$.

Fractional momentum deviation, $\delta=\left(p-p_{0}\right) / p_{0}(1-3)$.

Energy of a particle.

Nominal value of $E$.

Longitudinal electric field (3-8) and (c-8),

$y$-component of electric field (c-10).

Fractional energy deviation, $\varepsilon=\left(E-E_{0}\right) / E_{0}(1-3)$.

$\eta=1 / \gamma_{t}^{2}-1 / \frac{2}{\gamma}(1-4)$.

$\bar{n} \equiv n \omega_{0}^{2} /\left(\beta_{O}^{2} E_{0}\right)(1-4)$.

Azimuthal angular position relative to the reference particle, $\phi=\theta-\omega_{0} t(1-1)$.

Synchrotron amplitude $(6-8),(7-40 b)$, and (16-7).

$y$-component of Lorentz force (13-8) and (c-11).

Energy loss of particle per turn of revolution (7-27), $(7-28)$, and $(7-29)$.

Momentum distribution function normalized to 1 , $\int d \delta g(\delta)=1(4-3)$.

Energy in units of $\mathrm{mc}^{2}$.

Transition $\gamma(1-4)$.

Harmonic number of if (6-3). 


\begin{tabular}{|c|c|}
\hline$I_{a v}$ & Average current $(1-2)$. \\
\hline$I_{B}$ & Average current per bunch, $I_{B}=I_{a v} / h(11-16)$. \\
\hline$I(\theta, t)$ & Current at position $\theta$ and time $t(3-3)$. \\
\hline$I_{n}(\omega)$ & Fourier component of $I(\theta, t)(3-4)$ \\
\hline$I_{\mu}(x)$ & Modified Bessel function $(7-64)$. \\
\hline $\operatorname{Im}(x)$ & Imaginary part of $x$. \\
\hline $\mathrm{s}$ & Action variable of synchrotron motion $(7-40 \mathrm{~b})$. \\
\hline $\mathrm{J}_{\mathbf{s}}$ & Same as $J(16-7)$. \\
\hline$s_{y}$ & Action variable of $y$-betatron motion $(16-8)$. \\
\hline $\mathrm{k}_{\mathbf{s}}, \mathrm{R}_{\mathrm{y}}$ & $\begin{array}{l}\text { Rolmogorov-transformed action variables, conjugate } \\
\text { to } \alpha_{3} \text { and } \alpha_{y}(16-17,19) \text {. }\end{array}$ \\
\hline$\xi$ & Chromaticity, same as $\xi_{y}(14-5)$ \\
\hline$\lambda(\phi)$ & $\begin{array}{l}\text { Normalized unperturbed line density, } \int_{0}^{2 \pi} d \phi \lambda(\phi)=1 \\
(4-3) \text { and }(7-23) \text {. }\end{array}$ \\
\hline$\lambda_{\mathbf{n}}$ & Fourier component of $\lambda(\phi)(5-4)$. \\
\hline$\mu$ & $\begin{array}{l}\text { Harmonic number of synchrotron frequency }(7-64) \text { and } \\
(17-1) \text {. }\end{array}$ \\
\hline $\mathbf{N}$ & Total number of particles in the ring. \\
\hline n. & Harmonic number of revolution frequency (3-4). \\
\hline$\pi, n_{x}$ & $\begin{array}{l}\text { Focusing field index of a weak focusing machine (B-4) } \\
\text { and }(B-5) \text {. }\end{array}$ \\
\hline$\overline{\mathbf{n}}$ & $\bar{n}=n+\Omega / \omega_{0}(7-54)$ \\
\hline م & $\tilde{n}=n+a Q_{y o}(16-45)$. \\
\hline $\mathbf{p}$ & Momentum of particle. \\
\hline Po & Nominal value of $p$. \\
\hline$\phi$ & Azimuthal position relative to nominal particle $(1-1)$. \\
\hline$\psi$ & Angle-variable of synchrotron motion $(7-40 a)$. \\
\hline$\psi_{s}$ & Same as $\psi(16-6)$ \\
\hline
\end{tabular}




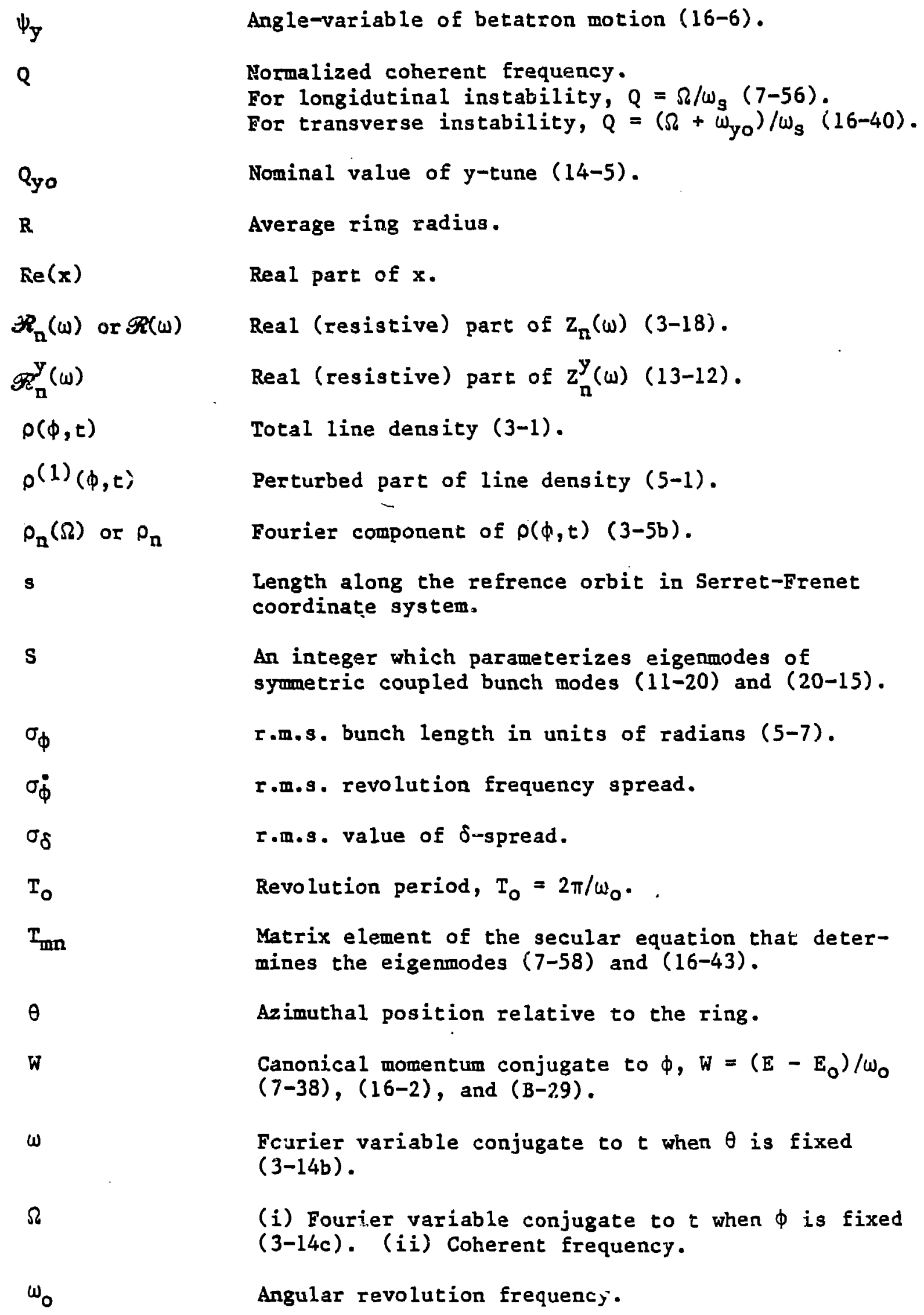

$\omega$

$\Omega$

$\omega_{0}$

Angle-variable of betatron motion (16-6).

Normalized coherent frequency.

For longidutinal instability, $Q=\Omega / \omega_{s}(7-56)$.

For transverse instability, $Q=\left(\Omega+\omega_{\text {yo }}\right) / \omega_{s}(16-40)$.

Nominal value of $y$-tune $(14-5)$.

Average ring radius.

Real part of $x$.

Real (resistive) part of $z_{n}(\omega)(3-18)$.

Real (resistive) part of $z_{n}^{y}(\omega)(13-12)$.

Total line density $(3-1)$.

Perturbed part of line density $(5-1)$.

Fourier component of $\rho(\phi, t)(3-5 b)$.

Length along the refrence orbit in Serret-Frenet coordinate system.

An integer which parameterizes eigenmodes of symmetric coupled bunch modes $(11-20)$ and $(20-15)$.

r.m.s. bunch length in units of radians (5-7).

r.m.s. revolution frequency spread.

r.m.s. value of $\delta$-spread.

Revolution period, $\mathrm{T}_{0}=2 \pi / \omega_{0}$. .

Matrix element of the secular equation that determines the eigenmodes $(7-58)$ and $(16-43)$.

Azimuthal position relative to the ring.

Canonical momentum conjugate to $\phi, W=\left(E-E_{0}\right) / \omega_{0}$ $(7-38),(16-2)$, and $(B-29)$.

Fcurier variable conjugate to $t$ when $\theta$ is fixed (3-14b).

(i) Fourier variable conjugate to $t$ when $\phi$ is fixed (3-14c). (ii) Coherent frequency.

Angular revolution frequency. 


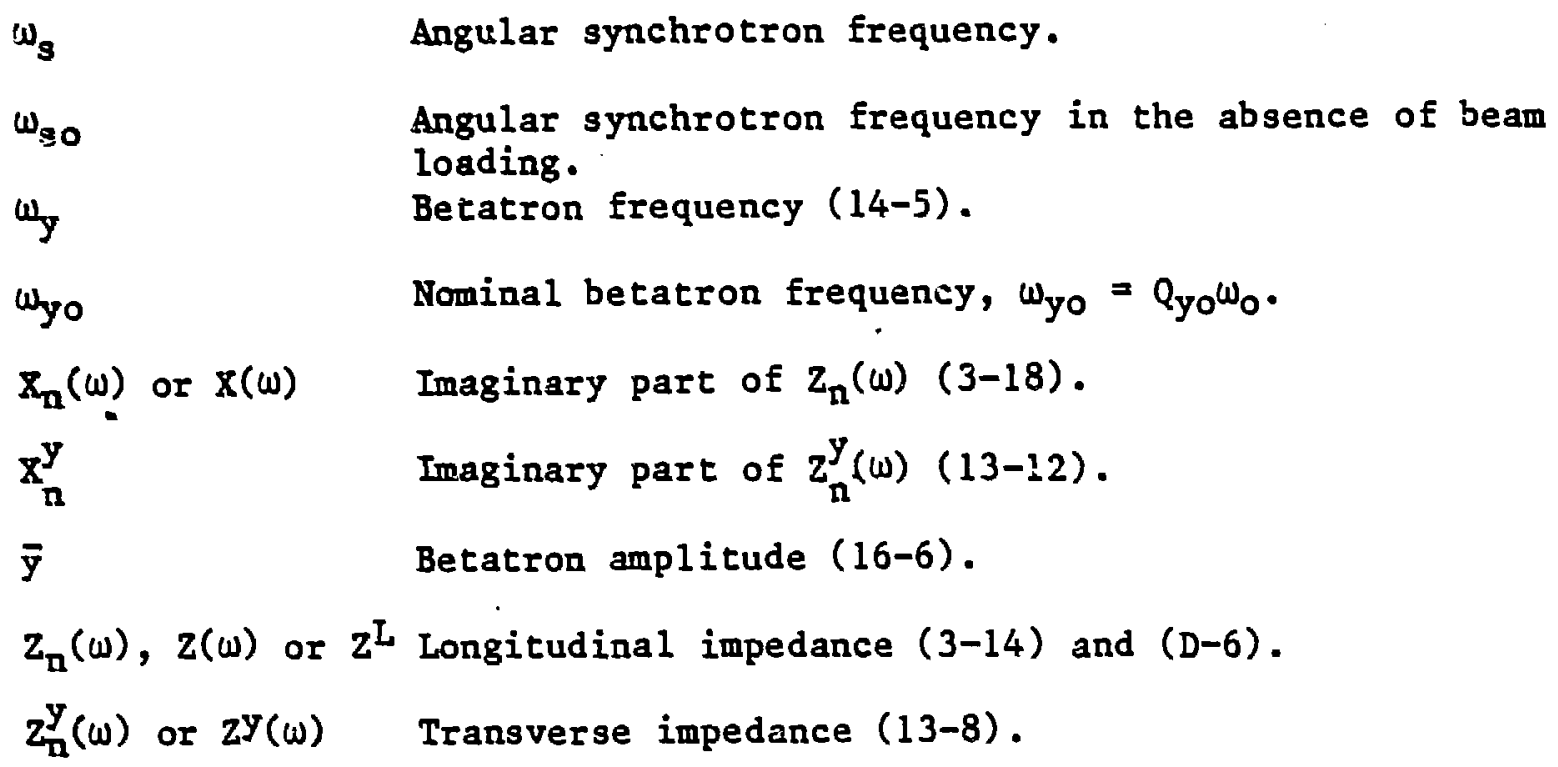

\section{ACRNOWLEDGMENT}

I thank Margaret Dienes for her careful and painstaking effort in editing these notes.

\section{REFERENCES}

1. C. Pellegrini, International School of Physics "Enrico Fermi," Academic Press, New York, 1971, p 221.

2. A. Hofmann, Proc. International School of Particle Accelerators, Erice, 1976, p 139.

3. B. Zotter and F. Sacherer, Ibid. P 175.

4. C. Pellegrini, 1981 Summer School on High Energy Particle Accelerators, AIP Proc. 87, p 78.

5. A. W. Chao, 1982 . Sumner School on High Energy Particle Accelerators, AIP Proc. 105, p 353.

6. E.D. Dourant and H.S. Snyder, Ann. Phys. 3, 1 (1958).

7. H. Bruck, Circular Particle Accelerators, English Transiations LASL, LA-TE-72-10, Rev. $197 z$.

3. J.D. Jackson, Classical Electrodynamics, 2nd ed., Wiley, New York, 1975.

9. D. Boussard, CERN LABII/RF/INT/75-2, 1975.

10. E. Messerschmid and M. Month, Nucl. Instrum. Methods 136, 1 (1976).

11. R. Robinson, CEAL Report TM-183 (1969).

12. P. Wilson, 1981 Sumer School on High Energy Particle Accelerators, AIP Proc. 87, P 450.

13. B.C. Craft, IEEE Trans. Nucl. Sci. NS-32, 2525 (1985).

14. I benefited from discussions with Ben Craft on Robinson instability.

15. J.M. Wang and C. Pellegrini, Proc. 11th Int. Conf. on High Energy Accelerators, Geneva, 1980, p 554. 
16. J.M. Wang and C. Pellegrini, Proc. 1979 Workshop on Beam Current Limitations in Storage Rings, BNL 51236, p 109.

17. R.D. Ruth and J.M. Wang, IEEE Trans. Nucl. Sci. NS-28, 2413 (1981).

18. R.D. Ruth, Ph.D. Thesis, BNL 51425, 1981.

19. S. Krinsky and J.M. Wang, Part. Accel. 17, 109 (1985); IEEE Trans. Nuc1. Sci. NS-20, 2498 (1983).

20. F. Sacherer, IEEE Trans. Nucl. Sci. NS-20, 825 (1973).

21. J.M. Wang, BNL 51302, 1980 .

22. K. Satoh, PEP-Note 357, 1981.

23. J. Bisognano, S. Chattopadhyay and J.M. Wang, to be published.

24. F.J. Sacherer, CERN/PS/BR/77-5, 1977.

25. R.D. Rohaupt, DESY $80 / 22,1980$.

26. R.D. Ruth and J.M. Wang, Unpublished, 1980.

27. R. Satoh, PEP Note 361, 1981.

28. A.M. Sessler and V. Vacaro, CERN/ISR/RF/67-2, 1967.

29. V.R. Neil and A.M. Sessler, Rev. Sci. Instr. 36, 429 (1965).

30. For an intuitive discussion of the Landau damping, see, e.g., H.G. Hereward, Proc. Int. School of Particle Accelerators, Erice, 1976, p 219.

31. A.G. Ruggiero and V.G. Vacaro, CERN ISR-TH/68-33, 1968.

32. E. Reil and W. Schnell, CERN ISE-TH-RF/69-48, 1969.

33. H. Goldstein, Classical Mechanisi, Addison-Wesley, 1950.

34. Discussion on Landau damping due to the synchrotron frequency spread can be found in Refs. 19, 21, and 23 .

35. E.D. Courant, IEEE Trans. Nucl. Sci. 12, 550 (1965).

36. C. Pellegrini, Nuovo Cimento 64, 447 (1969).

37. M. Sand, SLAC-TN-69-8, 1969.

38. W.R.H. Panofsky and W.A. Wentzel, Rev. Sci. Instr. 27, 967 (1956).

39. G. Nassibian and F. Sacherer, Nucl. Instrum. Methods 159, 21 (1979).

40. L.J. Laslett, V. R. Neil and A.M. Sessler, Rev. Sci. Instr. $\underline{36}$, 437 (1965).

41. We adopt the convention used in Ref. 33 in designating the generating functions $F_{1}, F_{2}, F_{3}$, and $F_{4}$ of canonical trans formations.

42. A.N. Rolmogorov, Dokl. Akad. Nauk SSSR 98, 527 (1954).

43. B.V. Chirikov, Phys. Rep. 52, 264 (1979).

44. E.D. Courant and A.M. Sessler, Rev. Sci. Instr. 37, 1579 (1966).

45. For example, see R.E. Collin, Foundations for Microwave Engineering, McGraw-Hi11, New York, 1966.

Research carried out under the auspices of the U.S.Department of Energy under Contract No. DE-AC02-76CH00016. 